\title{
The constant term of tempered functions on a real spherical space
}

\author{
Patrick Delorme* Bernhard Krötz Sofiane Souaifi
}

February 7, 2017

\begin{abstract}
Let $Z$ be a unimodular real spherical space which is assumed of wave-front type. Generalizing some ideas of Harish-Chandra [5, 6], we show the existence of the constant term for smooth tempered functions on $Z$, while Harish-Chandra dealt with $K$-finite functions on the group (see also the work of Wallach [16, Chapter 12], dealing with smooth functions on the group and using asymptotic expansions). By applying this theory, we get a characterization of the relative discrete series for $Z$. Some features for the constant term, namely transitivity and uniform estimates, are also established.
\end{abstract}

\section{Contents}

$\begin{array}{ll}\text { Introduction } & 1\end{array}$

1 Notation $\quad 5$

$2 \quad Z$-tempered $H$-fixed continuous linear forms and the space $\mathcal{A}_{\text {temp }}(Z) \quad 8$

2.1 Harish-Chandra representations of $G \ldots \ldots \ldots$

2.2 The spaces $C_{\text {temp }, N}^{\infty}(Z)$ and $\mathcal{A}_{\text {temp }, N}(Z) \ldots \ldots \ldots \ldots$

3 Differential equation for some functions on $Z$ wave-front and unimodular 15

3.1 Boundary degenerations of $Z \ldots \ldots \ldots \ldots$

3.2 Some estimates . . . . . . . . . . . . . . . . . . 15

3.3 Algebraic preliminaries . . . . . . . . . . . . . . . . . . . 18

3.4 The function $\varphi_{f}$ on $L_{I}$ and related differential equations . . . . . . . . . . 21

3.5 The function $\Phi_{f}$ on $A_{Z}$ and related differential equations . . . . . . . . . . 22

*The first author was supported by a grant of Agence Nationale de la Recherche with reference ANR-13BS01-0012 FERPLAY. 
4 Definition of the constant term and its properties $\quad 29$

4.1 Some estimates . . . . . . . . . . . . . . . . . . . . . . . 29

4.2 Definition of the constant term of elements of $\mathcal{A}_{\text {temp }}(Z: \mathcal{I}) \ldots \ldots 31$

4.3 Constant term of tempered $H$-fixed linear forms . . . . . . . . . . . . 36

4.4 Application to the relative discrete series for $Z \ldots \ldots$. . . . . . . . . 37

5 Proof of Proposition $4.11 \quad 39$

5.1 Reduction of the proof of Proposition 5.1 to the case where $Z$ is quasi-affine 39

5.2 Preliminaries to the proof of Proposition 5.1 when $Z$ is quasi-affine . . . . . 44

5.3 End of proof of Proposition 5.1 when $Z$ is quasi-affine . . . . . . . . . . 49

5.4 End of proof of Proposition $4.11 \ldots \ldots \ldots \ldots$

6 Transitivity of the constant term $\quad 55$

$\begin{array}{lll}7 & \text { Uniform estimates } & 56\end{array}$

A Variation of a Lemma due to N. Wallach 59

$\begin{array}{ll}\text { B Rapid convergence } & 61\end{array}$

\section{Introduction}

Let $Z=G / H$ be a real unimodular wave-front real spherical space. In this introduction $G$ is the group of real points of a connected reductive algebraic group $\underline{G}$ defined over $\mathbb{R}$, and $H$ is a connected subgroup of $G$ with algebraic Lie algebra such that there exists a minimal parabolic subgroup $P$ with $P H$ open in $G$.

The local structure theorem (cf. [10, Theorem 2.3]) associates a parabolic subgroup $Q$, said $Z$-adapted to $P$, with Levi decomposition $Q=L U$ (one has $P \subset Q$ ).

We will say that $A$ is a split torus of $G$ if it is the identity component of $\underline{A}(\mathbb{R})$, where $\underline{A}$ is a split $\mathbb{R}$-torus of $\underline{G}$.

Let $A_{L}$ be a maximal split torus of $L$ with Lie algebra $\mathfrak{a}_{L}$ and let $A_{H}$ be the analytic subgroup of $A_{L}$ with Lie algebra $\mathfrak{a}_{L} \cap \operatorname{Lie} H$. We choose a maximal split torus $A$ of $P \cap L$.

Then there exists a maximal compact subgroup $K$ of $G$ such that $G=K A N$ (resp. $L=$ $K_{L} A N_{L}$ ) is an Iwasawa decomposition of $G$ (resp. $L$, where $K_{L}=K \cap L$ and $N_{L}=N \cap L$ ). Let $M$ be the centralizer of $A$ in $K$.

Let $A_{Z}=A_{L} / A_{H}$. The (simple) spherical roots are defined in e.g. [11, Section 3.2]. They are real characters of $A_{Z}$ (or linear forms on $\mathfrak{a}_{Z}=$ Lie $A_{Z}$ ). Let $S$ be the set of spherical roots. Let $A_{Z}^{-}=\left\{a \in A_{Z}: a^{\alpha} \leqslant 1, \alpha \in S\right\}$. The polar decomposition asserts that there are two finite sets $\mathcal{F}$ and $\mathcal{W}$ of $G$ such that:

$$
Z=\mathcal{F} K A_{Z}^{-} \mathcal{W} \cdot z_{0},
$$

where $Q w H$ is open for each $w \in \mathcal{W}$ and $z_{0}$ denotes $H$ in the quotient space $Z$. In this paper, we make a certain choice of $\mathcal{W}$ (cf. Lemma 1.1). Let $\Omega=\mathcal{F} K$. 
Let $\rho_{Q}$ be the half sum of the roots of $\mathfrak{a}_{L}$ in Lie $U$. Actually $\rho_{Q} \in \mathfrak{a}_{Z}^{*}$.

For $f \in C^{\infty}(Z)$, we define:

$$
q_{N}(f)=\sup _{\omega \in \Omega, w \in \mathcal{W}, a \in A_{\bar{Z}}^{-}} a^{-\rho_{Q}}(1+\|\log a\|)^{-N}\left|f\left(\omega a w \cdot z_{0}\right)\right| .
$$

We define $C_{\text {temp,N }}^{\infty}(Z)$ as the space of $f \in C^{\infty}(Z)$ such that, for all $u$ in the enveloping algebra $U(\mathfrak{g})$ of the complexification $\mathfrak{g}_{\mathbb{C}}$ of $\mathfrak{g}=\operatorname{Lie} G$

$$
q_{N, u}(f):=q_{N}\left(L_{u} f\right)
$$

is finite. We endow $C_{t e m p, N}^{\infty}(Z)$ with the semi-norms $q_{N, u}$. Then $G$ acts in a $C^{\infty}$ way on $C_{\text {temp }, N}^{\infty}(Z)$. Let $(\pi, V)$ be a smooth Harish-Chandra $G$-representation. By this we mean the smooth Fréchet globalization with moderate growth of a $(\mathfrak{g}, K)$-module of finite length (see [2] or [16, Chapter 11]). Let $\mathcal{A}_{t e m p, N}(Z)$ be the subspace of elements of $C_{t e m p, N}^{\infty}(Z)$ which generate under the left regular representation a smooth Harish-Chandra $G$-sub-representation: this means that the closure of the linear span of their $G$-orbits is a Harish-Chandra $G$ representation. It is endowed with the topology induced by the topology of $C_{t e m p, N}^{\infty}(Z)$.

There is another definition of $\mathcal{A}_{t e m p, N}(Z)$. Let $\eta$ be a continuous $H$-fixed linear form on a Harish-Chandra $G$-representation $(\pi, V)$. One says that $\eta$ is $Z$-tempered if there exists $N \in \mathbb{N}$ such that, for all $v \in V$, the generalized matrix coefficient $m_{\eta, v}$, defined by:

$$
m_{\eta, v}(g)=<\eta, \pi\left(g^{-1}\right) v>, \quad g \in G,
$$

is in $C_{\text {temp }, N}^{\infty}(Z)$. Then one can show that $f \in \mathcal{A}_{\text {temp }, N}(Z)$ if and only if there exists such a $V$ and such an $\eta$ and $v_{0} \in V$ such that $f=m_{\eta, v_{0}}$.

Let $I$ be a subset of $S$ and let $\mathfrak{a}_{I}=\bigcap_{\alpha \in I} \operatorname{Ker} \alpha$. Let $X \in \mathfrak{a}_{I}^{--}=\left\{X \in \mathfrak{a}_{I}: \alpha(X)<0, \alpha \in\right.$ $S \backslash I\}$. Let $H_{I}$ be the analytic subgroup of $G$ with Lie algebra

$$
\text { Lie } H_{I}=\lim _{t \rightarrow+\infty} e^{t \text { ad } X} \text { Lie } H,
$$

where the limit is taken in the Grassmanian $G r(\mathfrak{g})$ of $\mathfrak{g}$. Then (see [11, Proposition 3.2]) $Z_{I}=G / H_{I}$ is a real spherical space, $P H_{I}$ is open in $G$ and $Q$ is $Z_{I}$-adapted to $P$. Let us denote $H_{I}$ by $z_{0, I}$ in the quotient space $Z_{I}$. Let $\mathcal{W}_{I}$ be the set corresponding to $\mathcal{W}$ for $Z_{I}$. One can define similarly $C_{\text {temp }, N}^{\infty}\left(Z_{I}\right)$ and $\mathcal{A}_{\text {temp }, N}\left(Z_{I}\right)$.

The main result of this paper is the following (cf. Proposition 4.8 and Theorem 4.13).

Theorem. Let $\mathcal{I}$ be a finite codimensional ideal of the center $Z(\mathfrak{g})$ of $U(\mathfrak{g})$ and let $\mathcal{A}_{\text {temp }, N}(Z: \mathcal{I})$ be the space of elements of $\mathcal{A}_{\text {temp,N }}(Z)$ annihilated by $\mathcal{I}$. There exists $N_{\mathcal{I}} \in \mathbb{N}$ such that, for all $N \in \mathbb{N}$, for each $f \in \mathcal{A}_{\text {temp }, N}(Z: \mathcal{I})$, there exists a unique $f_{I} \in \mathcal{A}_{\text {temp }, N+N_{\mathcal{I}}}\left(Z_{I}: \mathcal{I}\right)$ such that, for all $g \in G, X \in \mathfrak{a}_{I}^{--}$:

(i) $\lim _{T \rightarrow+\infty} e^{-T \rho_{Q}(X)}\left(f(g \exp (T X))-f_{I}(g \exp (T X))\right)=0$.

(ii) $T \mapsto e^{-T \rho_{Q}(X)} f_{I}(g \exp (T X))$ is an exponential polynomial with unitary characters, i.e. of the form $\sum_{j=1}^{n} p_{j}(T) e^{i \nu_{j} T}$, where the $p_{j}$ 's are polynomials and the $\nu_{j}$ 's are real numbers. 
Moreover the linear map $f \mapsto f_{I}$ is a continuous $G$-morphism and, for each $w_{I} \in \mathcal{W}_{I}$, there exist $w \in \mathcal{W}, m_{w_{I}} \in M$ such that, for any compact subset $\mathcal{C}$ in $\mathfrak{a}_{I}^{--}$and any compact subset $\Omega$ of $G$, there exists $\varepsilon>0$ and a continuous semi-norm $p$ on $\mathcal{A}_{\text {temp }, N}(Z)$ such that:

$$
\begin{aligned}
& \left|(a \exp T X)^{-\rho_{Q}}\left(f\left(\omega a \exp (T X) w \cdot z_{0}\right)-f_{I}\left(\omega m_{w_{I}}^{-1} a \exp (T X) w_{I} \cdot z_{0, I}\right)\right)\right| \\
& \leqslant e^{-\varepsilon T} p(f)(1+\|\log a\|)^{N}, \quad a \in A_{Z}^{-}, X \in \mathcal{C}, \omega \in \Omega, T \geqslant 0 .
\end{aligned}
$$

This generalizes the work of Harish-Chandra in the group case (see [5, Sections 21 to 25], also the work of Wallach [16, Chapter 12]) and the one of Carmona for symmetric spaces (see [4]). A certain control of these estimates are established when $\mathcal{I}$ is the kernel of a character of $Z(\mathfrak{g})$ and varies in such a way that (in particular) the real part of the Harish-Chandra parameter of this character is fixed (see Theorem 7.4 for more detail). This is related to some results of Harish-Chandra (cf. [6, Section 10]).

While the work of Harish-Chandra is for $K$-finite functions, we deal with smooth tempered functions, but without using asymptotic expansions as it is done in [16, Chapter 12].

For a $Z$-tempered continuous linear form $\eta$ on a Harish-Chandra $G$-representation $(\pi, V)$, one can define a constant term $\eta_{I}$ which is a $Z_{I}$-tempered continuous linear form on $V$ in such a way that, for all $v \in V$,

$$
m_{\eta_{I}, v}\left(z_{I}\right)=\left(m_{\eta, v}\right)_{I}\left(z_{I}\right), \quad z_{I} \in Z_{I}
$$

(cf. Proposition 4.14). Moreover we show that, if $(\pi, V)$ is irreducible with unitary central character, then $(\pi, V, \eta)$ is a discrete series modulo the center of $Z$ if and only if for all $I \mp S$, $\eta_{I}=0$ (see Theorem 4.15). Again it is analogous to a result of Harish-Chandra. For this we use in a crucial manner some results on discrete series from [11, Section 8]. More generally, our work owes a lot to their work.

The proof of these results is quite parallel to the work of Harish-Chandra on the constant term (cf. [5,6]) by studying certain system of linear differential equations. In the case of one variable, this reduces to show the following:

Let $E$ be a finite dimensional complex vector space, $A \in \operatorname{End}(E), \psi \in$ $C^{\infty}([0,+\infty[, E)$ of exponential decay, i.e.

$$
\text { there exists } \beta<0 \text { such that }\|\psi(t)\| \leqslant e^{\beta t}, t \geqslant 0 .
$$

Consider the linear differential equation on $[0,+\infty[$ :

$$
\phi^{\prime}=A \phi+\psi
$$

Then, if $\phi$ is a bounded solution, there exists an exponential polynomial $\tilde{\phi}$ with unitary characters such that:

$$
\lim _{t \rightarrow \infty} \phi(t)-\tilde{\phi}(t)=0
$$


There are some variations, as we are allowed to work with vectors in a Harish-Chandra $G$-representation, where Harish-Chandra was working only with $K$-finite functions. Some important properties of Harish-Chandra $G$-representations are used (see e.g. [16, Chapter 11] or [2]).

First one establishes the Theorem for $w_{I}=1$. The passage to general $w_{I}$ is delicate. One has to give some more insight on the link between $w$ and $w_{I}$ explained in [11, Lemma 3.10]. This is done in Proposition 5.1 which holds for general spherical spaces. It uses a reduction to quasi-affine spherical spaces and properties of finite dimensional representations.

The motivation of our work is the determination of the Plancherel formula for $Z$ along the lines of the work of Sakellaridis and Venkatesh (cf. [13]). This requires several important changes as it is quite unclear what could be the asymptotics for general $C^{\infty}$, even $K$-finite, functions. We hope that our results will allow to avoid these asymptotics.

\section{Notation}

In this paper, we will denote (real) Lie groups by upper case Latin letters and their Lie algebras by lower case German letters. If $R$ is a real Lie group, then $R_{0}$ will denote its identity component.

Let $\underline{G}$ be a connected reductive algebraic group defined over $\mathbb{R}$ and let $\underline{G}(\mathbb{R})$ be its group of real points. Let $G$ be an open subgroup of the real Lie group $\underline{G}(\mathbb{R})$.

If $R$ is a closed subgroup of $G$, we will denote by $R_{\mathbb{C}, 0}$ the connected analytic subgroup of $\underline{G}(\mathbb{C})$ with Lie algebra $\mathfrak{r}_{\mathbb{C}}$. Then we set $R_{0}=R_{\mathbb{C}, 0} \cap G$. Note that:

$$
\text { if } R \text { is a Levi subgroup of } G \text { then } R \subset R_{\mathbb{C}, 0} \text {, }
$$

as $R_{\mathbb{C}, 0}$ is a Levi subgroup of $\underline{G}(\mathbb{C})$ (remark that Levi subgroups of a complex group are connected).

We will say that $A$ is a split torus of $G$ if it is of the form $\underline{A}(\mathbb{R})_{0}$, where $\underline{A}$ is an $\mathbb{R}$-split torus of $\underline{G}$.

Let $H$ be a closed connected subgroup of $G$ such that $\mathfrak{h}$ is algebraic, and let us assume that $Z=G / H$ is real spherical. This means that there exists a minimal parabolic subgroup $P$ of $G$ with $P H$ open in $G$.

From the local structure theorem (cf. [10, Theorem 2.3]),

There exists a unique parabolic subgroup $Q$ of $G$ with a Levi decomposition $Q=L U$ such that:

(i) $P \cdot z_{0}=Q \cdot z_{0}$,

(ii) $L_{n} \subset Q \cap H \subset L$,

where $z_{0}$ denotes $H$ in $Z$ and $L_{n}$ is the product of all non compact non abelian factors in $L$. 
Such a parabolic subgroup $Q$ is called $Z$-adapted to $P$. Let $A_{L}$ be a maximal split torus of the center of $L$ and $A_{H}=\left(A_{L} \cap H\right)_{0}$. Let $A$ be a maximal split torus of $P \cap L$. It contains $A_{L}$.

Let us prove that there exist a maximal compact subgroup $K$ of $G$ and an involution $\theta$ of $G$ such that its differential, denoted also by $\theta$, restricted to $[\mathfrak{g}, \mathfrak{g}]$, is equal to the Cartan involution associated to $\mathfrak{k} \cap[\mathfrak{g}, \mathfrak{g}], \theta(X)=X$ if $X \in \mathfrak{c} \cap \mathfrak{k}$, where $\mathfrak{c}$ is the center of $\mathfrak{g}$, and $\theta(X)=-X$ if $X \in \mathfrak{a}$.

First one notices that $A$ contains a maximal split torus $A_{G}$ of the center of $G$. It is, in the terminology of [5] or [14, p. 197], a split component of $G$. In fact, one can construct a maximal split torus of $G$ by starting with a maximal split torus of the derived group $G_{1}$ of $G_{0}$, which has this property. But all maximal split tori of $G$ are conjugate by an element of $G$ as it is the case for maximal $\mathbb{R}$-split tori of $\underline{G}(\mathbb{R})$ (cf. [3, Theorem 20.9]). Hence $A$ has also the required property and one has $\mathfrak{a}=\mathfrak{a}_{1} \oplus \mathfrak{a}_{G}$, where $\mathfrak{a}_{1}=\mathfrak{a} \cap[\mathfrak{g}, \mathfrak{g}]$.

Now we can find $\left(K^{\prime}, \theta^{\prime}, \mathfrak{a}^{\prime}\right)$ with the above properties when replacing $K$ by $K^{\prime}, \theta$ by $\theta^{\prime}$, $\mathfrak{a}$ by $\mathfrak{a}^{\prime}$ and such that $\mathfrak{a}^{\prime}$ contains $\mathfrak{a}_{G}$ (cf. [14, Part II, Section 1, Theorem 3.13]), but we do not require $\mathfrak{a}^{\prime}$ to be the Lie algebra of a maximal split torus of $G$. Let $\mathfrak{j}_{1}$ (resp. $\mathfrak{j}_{1}^{\prime}$ ) be a Cartan subalgebra of $Z_{[\mathfrak{g}, \mathfrak{g}]}\left(\mathfrak{a}_{1}\right)$ (resp. $Z_{[\mathfrak{g}, \mathfrak{g}]}\left(\mathfrak{a}_{1}^{\prime}\right)$, where $\mathfrak{a}_{1}^{\prime}=\mathfrak{a}^{\prime} \cap[\mathfrak{g}, \mathfrak{g}]$ ). Then $\mathfrak{j}_{1}$ and $\mathfrak{j}_{1}^{\prime}$ are maximally split Cartan subalgebras of $[\mathfrak{g}, \mathfrak{g}]$, hence there are conjugate by an element $g$ of $G_{1}$. As $\mathfrak{a}_{1}$ (resp. $\left.\mathfrak{a}_{1}^{\prime}\right)$ is equal to the space of $X \in \mathfrak{j}_{1}$ (resp. $\left.\mathfrak{j}_{1}^{\prime}\right)$ such that the eigenvalues of $\operatorname{ad}_{[\mathfrak{g}, \mathfrak{g}]} X$ are real, the element $g$ conjugates $\mathfrak{a}_{1}$ and $\mathfrak{a}_{1}^{\prime}$, i.e. $\operatorname{Ad}(g) \mathfrak{a}_{1}=\mathfrak{a}_{1}^{\prime}$. Hence $\operatorname{Ad}(g) \mathfrak{a}=\mathfrak{a}^{\prime}$. Then $K=g K^{\prime} g^{-1}$ and $\theta=\theta^{\prime} \circ \operatorname{Ad}\left(g^{-1}\right)$ satisfy the required properties and $G=K A N$ is an Iwasawa decomposition.

Moreover, as $L=Z_{G}\left(A_{L}\right)$ and $A_{L} \subset A$ is $\theta$-stable, $L$ is $\theta$-stable and $L=K_{L} A N_{L}$ is an Iwasawa decomposition, where $K_{L}=K \cap L$ and $N_{L}=N \cap L$.

Let $A_{Z}=A_{L} / A_{H}$. Let us notice, from the fact that $L_{n} \subset L \cap H$, that $\mathfrak{a}_{Z}=\mathfrak{a} / \mathfrak{a} \cap \mathfrak{h}$.

We choose a section $s: A_{Z} \rightarrow A_{L}$ of the projection $A_{L} \rightarrow A_{L} / A_{H}$ which is a

morphism of Lie groups. We will often use $\tilde{a}$ instead of $s(a)$.

Let $B$ be a $\mathfrak{g}, \operatorname{Ad} G$ and $\theta$-invariant bilinear form on $\mathfrak{g}$ such that the quadratic form $X \mapsto$ $\|X\|^{2}=-B(X, \theta X)$ is positive definite. We will denote by $(\cdot, \cdot)$ the corresponding scalar product on $\mathfrak{g}$. It defines a quotient scalar product and a quotient norm on $\mathfrak{a}_{Z}$ that we still denote by $\|\cdot\|$.

Let $\Sigma$ be the set of roots of $\mathfrak{a}$ in $\mathfrak{g}$. If $\alpha \in \Sigma$, let $\mathfrak{g}^{\alpha}$ be the corresponding weight space for $\mathfrak{a}$. We write $\Sigma_{\mathfrak{u}}\left(\right.$ resp. $\left.\Sigma_{\mathfrak{n}}\right) \subset \Sigma$ for the set of $\mathfrak{a}$-roots in $\mathfrak{u}$ (resp. $\left.\mathfrak{n}\right)$ and set $\mathfrak{u}^{-}=\sum_{\alpha \in \Sigma_{\mathfrak{u}}} \mathfrak{g}^{-\alpha}$, i.e. the nilradical of the parabolic subalgebra $\mathfrak{q}^{-}$opposite to $\mathfrak{q}$ with respect to $\mathfrak{a}$.

Let $(\mathfrak{l} \cap \mathfrak{h})^{\perp_{\mathfrak{l}}}$ be the orthogonal of $\mathfrak{l} \cap \mathfrak{h}$ in $\mathfrak{l}$ with respect to the scalar product $(\cdot, \cdot)$. One has:

$$
\mathfrak{g}=\mathfrak{h} \oplus(\mathfrak{l} \cap \mathfrak{h})^{\perp \mathfrak{l}} \oplus \mathfrak{u}
$$

Let $T$ be the restriction to $\mathfrak{u}^{-}$of minus the projection from $\mathfrak{g}$ onto $(\mathfrak{l} \cap \mathfrak{h})^{\perp} \oplus \mathfrak{u}$ parallel to $\mathfrak{h}$. Let $\alpha \in \Sigma_{\mathfrak{u}}$ and $X_{-\alpha} \in \mathfrak{g}^{-\alpha}$. Then (cf. [11, equation (3.2)])

$$
T\left(X_{-\alpha}\right)=\sum_{\beta \in \Sigma_{\mathfrak{u}} \cup\{0\}} X_{\alpha, \beta},
$$


with $X_{\alpha, \beta} \in \mathfrak{g}^{\beta} \subset \mathfrak{u}$ if $\beta \in \Sigma_{\mathfrak{u}}$ and $X_{\alpha, 0} \in(\mathfrak{l} \cap \mathfrak{h})^{\perp_{\mathfrak{r}}}$.

Let $\mathcal{M} \subset \mathbb{N}_{0}\left[\Sigma_{\mathfrak{u}}\right]$ be the monoid generated by:

$$
\left\{\alpha+\beta: \alpha \in \Sigma_{\mathfrak{u}}, \beta \in \Sigma_{\mathfrak{u}} \cup\{0\} \text { such that there exists } X_{-\alpha} \in \mathfrak{g}^{-\alpha} \text { with } X_{\alpha, \beta} \neq 0\right\} .
$$

The elements of $\mathcal{M}$ vanish on $\mathfrak{a}_{H}$ so $\mathcal{M}$ identifies to a subset of $\mathfrak{a}_{Z}^{*}$. We define

$$
\begin{aligned}
& \mathfrak{a}_{Z}^{--}=\left\{X \in \mathfrak{a}_{Z}: \alpha(X)<0, \alpha \in \mathcal{M}\right\} \\
& \text { and } \quad \mathfrak{a}_{Z}^{-}=\left\{X \in \mathfrak{a}_{Z}: \alpha(X) \leqslant 0, \alpha \in \mathcal{M}\right\} .
\end{aligned}
$$

Following e.g. [11], we define the set $S$ of spherical roots as the set of irreducible elements of $\mathcal{M}$, i.e. those which cannot be expressed as a sum of two non-zero elements in $\mathcal{M}$. We define also

$$
\mathfrak{a}_{Z, E}=\left\{X \in \mathfrak{a}_{Z}: \alpha(X)=0, \alpha \in S\right\}
$$

which normalizes $\mathfrak{h}$.

We have the polar decomposition for $Z$. Namely (cf. [11, equation (3.16)] or [8, Theorem 5.13]),

There exist two finite sets $\mathcal{F}^{\prime \prime}$ and $\mathcal{W}$ in $G$ such that $Z=\mathcal{F}^{\prime \prime} K A_{Z}^{-} \mathcal{W} \cdot z_{0}$ and such that $P w H$ is open and $A_{H} w \subset w H$ for each $w \in \mathcal{W}$.

Moreover

Any open $(P, H)$-orbit in $G$ is of the form $P w H$ for at least an element $w$ of $\mathcal{W}$.

Let us recall some notation used in [11, Section 3.4]. Let

$$
\hat{\mathfrak{h}}:=\mathfrak{h}+\tilde{\mathfrak{a}}_{Z, E},
$$

let $\widehat{H}_{\mathbb{C}, 0}$ be the connected algebraic subgroup of $G_{\mathbb{C}}$ with Lie algebra $\hat{\mathfrak{h}}_{\mathbb{C}}, \widehat{H}_{0}:=\widehat{H}_{\mathbb{C}, 0} \cap G$ and $T_{Z}:=\exp \left(i \mathfrak{a}_{Z}\right)$. Recall that $\mathfrak{h}$ is an ideal in $\hat{\mathfrak{h}}$. Then $\hat{H}_{\mathbb{C}, 0}=\exp \left(i \tilde{\mathfrak{a}}_{Z, E}\right) \tilde{A}_{Z, E} H_{\mathbb{C}, 0}$.

1.1 Lemma. The set $\mathcal{W}$ can be chosen such that any $w \in \mathcal{W}$ can be written:

$$
w=t h \text {, where } t \in \exp \left(i \tilde{\mathfrak{a}}_{Z}\right) \text { and } h \in H_{\mathbb{C}, 0} .
$$

Moreover, if $a \in A_{H}, a w \cdot z_{0}=w \cdot z_{0}$.

Proof. Let us use the notation of [11, after equation (3.12)]. Any $f \in \mathcal{F}$ can be written $f=t h$ with $h \in \widehat{H}_{\mathbb{C}, 0}=\exp \left(i \tilde{\mathfrak{a}}_{Z, E}\right) \widetilde{A}_{Z, E} H_{\mathbb{C}, 0}$ and $t \in T_{Z}$. Then write $h=a_{Z, E} t_{Z, E} h_{1}$ with $h_{1} \in H_{\mathbb{C}, 0}, a_{Z, E} \in \widetilde{A}_{Z, E}, t_{Z, E} \in \widetilde{T}_{Z, E}=\exp \left(i \tilde{\mathfrak{a}}_{Z, E}\right)$. As $a_{Z, E} \in P$, one is allowed to change $w$ in $a_{Z, E}^{-1} w$ in loc.cit. equation (3.12). Hence, elements of this chosen set $\mathcal{F}$ satisfies (1.6), i.e.

$$
f=t h, \quad t \in \exp \left(i \tilde{\mathfrak{a}}_{Z}\right), h \in H_{\mathbb{C}, 0} .
$$


Now $\mathcal{W}=\mathcal{F} \mathcal{F}^{\prime}$ (cf. loc.cit. after equation (3.15)) and $\mathcal{F}^{\prime}$ is a finite subset of $\widehat{H}_{0}=\widehat{H}_{\mathbb{C}, 0} \cap G \subset$ $N_{G}(H)$ (cf. loc.cit. equation (3.14)). More precisely, $\mathcal{F}^{\prime}$ is a minimal set of representatives of $\hat{H}_{0} / H A_{Z, E}$. Let us first study elements $f^{\prime}$ of $\mathcal{F}^{\prime} \subset \hat{H}_{0}$. These elements can be written $f^{\prime}=a_{Z, E}^{\prime} t_{Z, E}^{\prime} h_{1}^{\prime}$ with $a_{Z, E}^{\prime} \in \widetilde{A}_{Z, E}, t_{Z, E}^{\prime} \in \widetilde{T}_{Z, E}, h_{1}^{\prime} \in H_{\mathbb{C}, 0}$. Hence, using (1.7), $a_{Z, E}^{\prime-1} f f^{\prime}=$ $t t^{\prime} h_{1} h_{1}^{\prime}$, where $h_{1}\left(a_{Z, E}^{\prime}\right)^{-1}\left(t_{Z, E}^{\prime}\right)^{-1} h\left(t_{Z, E}^{\prime}\right)^{-1} a_{Z, E}^{\prime} \in H_{\mathbb{C}, 0}$, as $\widetilde{A}_{Z, E}$ and $\widetilde{T}_{Z, E}$ normalize $H_{\mathbb{C}, 0}$. Then, by changing the element $f f^{\prime}$ into $a_{Z, E}^{\prime-1} f f^{\prime}$, we define a new choice $\mathcal{W}$ for which the polar decomposition (1.5) is valid and its elements satisfy (1.6).

The elements of the original $\mathcal{W}$ satisfy $a w \cdot z_{0}=w \cdot z_{0}$ (cf. [11, Lemma 3.5 and its proof $]$ ). As the elements of the new set $\mathcal{W}$ are obtained by multiplying the elements of the old one by elements of $\widetilde{A}_{Z, E}$ which commute to $A_{H}$, one gets the last assertion of the Lemma.

If $w \in \mathcal{W}$, one introduces $H_{w}=w H w^{-1}$ and $Z_{w}=G / H_{w}$. Then (cf. [11, Corollary 3.7]), $P H_{w}$ is open and $Q$ is $Z_{w}$-adapted to $P$. Moreover $A_{Z_{w}}=A_{Z}$ and $A_{Z_{w}}^{-}=A_{Z}^{-}$. Let $\Omega$ denote the compact set $\mathcal{F} K$.

\section{$2 Z$-tempered $H$-fixed continuous linear forms and the space $\mathcal{A}_{\text {temp }}(Z)$}

\subsection{Harish-Chandra representations of $G$}

Let us recall some definitions and results of [2].

A continuous representation $(\pi, E)$ of a Lie group $G$ on a topological vector space $E$ is a representation such that the map:

$$
G \times E \rightarrow E,(g, v) \mapsto \pi(g) v, \text { is continuous. }
$$

If $R$ is a compact subgroup of $G$ and $v \in E$, we say that $v$ is $R$-finite if $\pi(R) v$ generates a finite dimensional subspace of $E$. Let $V_{(R)}$ denote the vector space of $R$-finite vectors in $E$. Let $\eta$ be a continuous linear form on $E$ and $v \in E$. Let us define the generalized matrix coefficient associated to $\eta$ and $v$ by:

$$
m_{\eta, v}(g):=<\eta, \pi\left(g^{-1}\right) v>, \quad g \in G .
$$

Let $G$ be a real reductive group. Let $\|\cdot\|$ be a norm on $G$ (cf. [15, Section 2.A.2] or [2, Section 2.1.2]). We have the notion of a Fréchet representation with moderate growth. A representation $(\pi, E)$ of $G$ is called a Fréchet representation with moderate growth if it is continuous and if for any continuous semi-norm $p$ on $E$, there exist a continuous semi-norm $q$ on $E$ and $N \in \mathbb{N}$ such that:

$$
p(\pi(g) v) \leqslant q(v)\|g\|^{N}, \quad v \in E, g \in G .
$$

This notion coincides with the notion of F-representations given in [2, Definition 2.6] for the large scale structure corresponding to the norm $\|\cdot\|$. We will adopt the terminology of F-representations. 
Let $(\pi, E)$ be an F-representation. A smooth vector in $E$ is a vector such that $g \mapsto \pi(g) v$ is smooth from $G$ to $E$. The space $V^{\infty}$ of smooth vectors in $V$ is endowed with the Sobolev semi-norms that we define now. Fix a basis $X_{1}, \ldots, X_{n}$ of $\mathfrak{g}$ and $k \in \mathbb{N}$. Let $p$ be a continuous semi-norm on $E$ and set

$$
p_{k}(v)=\left(\sum_{m_{1}+\cdots+m_{n} \leqslant k} p\left(\pi\left(X_{1}^{m_{1}} \cdots X_{n}^{m_{n}}\right) v\right)^{2}\right)^{1 / 2}, \quad v \in E^{\infty} .
$$

We endow $E^{\infty}$ with the topology defined by the semi-norms $p_{k}, k \in \mathbb{N}$, when $p$ varies in the set of continuous semi-norms of $E$, and denote by $\left(\pi^{\infty}, E^{\infty}\right)$ the corresponding sub-representation of $(\pi, E)$.

An SF-representation is an F-representation $(\pi, E)$ which is smooth, i.e. such that $E=$ $E^{\infty}$ as topological vector spaces. Let us remark that if $(\pi, E)$ is an F-representation, then $\left(\pi^{\infty}, E^{\infty}\right)$ is an SF-representation (cf. [2, Corollary 2.16]). The topology on $E^{\infty}$ is also given by the semi-norms:

$$
\Delta_{p_{2 k}}(v)=\left(\sum_{j=0}^{k}\left(p\left(\pi\left(\Delta^{j}\right) v\right)\right)^{2}\right)^{1 / 2}, \quad v \in E^{\infty},
$$

where $\Delta=X_{1}^{2}+\cdots+X_{n}^{2}$ and $p$ varies in the set of continuous semi-norms of $E$.

2.1 Lemma. Let $G$ be a real reductive group and $K$ be a maximal compact subgroup of $G$. Let $(\pi, E)$ be a continuous Banach representation of $G$ (i.e. a continuous representation in a Banach space).

(i) Let $V$ be a $(\mathfrak{g}, K)$-module of finite length which is contained in $E^{\infty}$. Then $V$ is contained in the space $E^{\omega}$ of analytic vectors of $E$.

(ii) The closure of $V$ in $E^{\infty}, \bar{V}$, is an $S F$-representation of $G$ with underlying $(\mathfrak{g}, K)$-module equal to $V$. In fact $\bar{V}$ is isomorphic to the canonical SF-globalization of $V$.

Proof. Let $C_{\mathfrak{g}}$ be the Casimir element of $U(\mathfrak{g})$ and let $C_{\mathfrak{k}}$ be the Casimir element of $U(\mathfrak{k})$. Then $\Delta:=C_{\mathfrak{g}}-2 C_{\mathfrak{k}}$ is a Laplacian for $G$. Since $V$ is of finite length, every element of $V$ is a finite linear combination of $v \in V$ satisfying the following:

There exist $\Lambda_{\mathfrak{g}}, \Lambda_{\mathfrak{k}} \in \mathbb{C}$ and $n \in \mathbb{N}$ such that $\pi\left(C_{\mathfrak{g}}-\Lambda_{\mathfrak{g}}\right)^{n} v=0$ and $\pi\left(C_{\mathfrak{k}}-\Lambda_{\mathfrak{k}}\right)^{n} v=$ 0 .

This implies that, if $\Lambda=\Lambda_{\mathfrak{g}}-2 \Lambda_{\mathfrak{k}}$,

$$
\pi(\Delta-\Lambda)^{2 n} v=0 .
$$

To show that $V \subset E^{\omega}$, it is then enough to show that $v \in E^{\omega}$ for such $v$. Fix such a $v \in V$. Let $\eta$ be a continuous linear form on $E$. Then the generalized matrix coefficient $m_{\eta, v}$ is a smooth function on $G$, as $v \in V \subset E^{\infty}$, and is annihilated by $(\Delta-\Lambda)^{2 n}$. Hence $m_{\eta, v}$ is analytic. This shows that:

$$
\begin{aligned}
G & \rightarrow E \\
g & \mapsto \pi(g) v
\end{aligned} \text { is weakly analytic. }
$$


As $E$ is a Banach space, it follows from [17, Lemma 4.4.5.1] that the map is analytic. Hence $v \in V^{\omega}$ and (i) follows.

Let us show (ii). We first prove that $\bar{V}$ is $G$-invariant. It is clearly $K$-invariant as $V$ is. It is also invariant by the identity component of $G$ due to [17, Corollary 4.4.5.5]. Hence it is $G$-invariant. Then $\bar{V}$ is a closed $G$-submodule of $E^{\infty}$, hence of moderate growth as $E$ is a continuous Banach representation of $G$. It remains to check that $V$ is equal to the space of $K$-finite elements in $V^{\infty}$. Let $v$ be a $K$-finite element of $\bar{V}$. Let us prove that $v \in V$. By linearity, one can assume that there exists a finite dimensional representation of $K, \delta$, with normalized character $\chi_{\delta}$, such that:

$$
\pi\left(\chi_{\delta}\right) v=v .
$$

On the other hand, $v$ is the limit of a sequence $\left(v_{n}\right)$ of elements of $V$. Hence $\pi\left(\chi_{\delta}\right) v_{n} \underset{n \rightarrow+\infty}{\longrightarrow}$ $\pi\left(\chi_{\delta}\right) v=v$. But $\left(\pi\left(\chi_{\delta}\right) v_{n}\right)_{n \in \mathbb{N}}$ lies in a finite dimensional subspace of $V$. Hence $v$ belongs to this finite dimensional subspace of $V$. In particular $v \in V$. This achieves to prove the Lemma.

We define a Harish-Chandra representation of $G$ as an SF-representation $V^{\infty}$ such that the underlying $(\mathfrak{g}, K)$-module of $K$-finite vectors $V$ is of finite length.

\subsection{The spaces $C_{t e m p, N}^{\infty}(Z)$ and $\mathcal{A}_{\text {temp }, N}(Z)$}

In the remaining of Section 2, we will assume that $Z$ is unimodular. Let $\rho_{Q}$ be the half sum of the roots of $\mathfrak{a}$ in $\mathfrak{u}$. Let us show that:

$$
\rho_{Q} \text { is trivial on } \mathfrak{a}_{H} .
$$

As $\mathfrak{l} \cap \mathfrak{h}$-modules,

$$
\mathfrak{g} / \mathfrak{h}=\mathfrak{u} \oplus(\mathfrak{l} / \mathfrak{l} \cap \mathfrak{h}) .
$$

But the action of $\mathfrak{a}_{H}=\mathfrak{a}_{L} \cap \mathfrak{h}$ on $(\mathfrak{l} / \mathfrak{l} \cap \mathfrak{h})$ is trivial. Since $Z$ is unimodular, the action of $\mathfrak{a}_{H}$ has to be unimodular. Our claim follows.

Hence $\rho_{Q}$ can be defined as a linear form on $\mathfrak{a}_{Z}$.

We have the notion of weights on an homogeneous space $X$ of a locally compact group $G$ (cf. [1, Section 3.1]). This is a function $w: X \rightarrow \mathbb{R}^{+*}$ such that, for every ball $B$ of $G$ (i.e. a compact symmetric neighborhood of 1 in $G)$, there exists a constant $c=c(w, B)$ such that:

$$
w(g \cdot x) \leqslant c w(x), \quad g \in B, x \in X .
$$

One sees easily that if $w$ is a weight, then $w^{-1}$ is also a weight.

Let $\mathbf{v}$ (resp. w) be the weight function on $Z$ defined in [8, Section 4] (resp. [8, Proposition 3.4]). For any $N \in \mathbb{N}$, let $E_{N}$ be the completion of $C_{c}^{\infty}(Z)$ for the norm $p_{N}$ defined by:

$$
p_{N}(f)=\sup _{z \in Z}\left((1+\mathbf{w}(z))^{-N} \mathbf{v}(z)^{1 / 2}|f(z)|\right),
$$


i.e. $E_{N}$ consists of the space of continuous functions $f$ on $Z$ such that $p_{N}(f)<+\infty$. From the polar decomposition of $Z$ (cf. (1.5)), one has:

$$
p_{N}(f)=\sup _{\omega \in \Omega, a \in A_{Z}^{-}, w \in \mathcal{W}}\left(\left(1+\mathbf{w}\left(\omega a w \cdot z_{0}\right)\right)^{-N} \mathbf{v}\left(\omega a w \cdot z_{0}\right)^{1 / 2}\left|f\left(\omega a w \cdot z_{0}\right)\right|\right) .
$$

From the fact that $\mathbf{v}$ and $\mathbf{w}$ are weight functions on $Z$ and from [8, Propositions 3.4(2) and 4.3], one then sees that:

The norm $p_{N}$ is equivalent to the norm:

$$
f \mapsto q_{N}(f):=\sup _{\omega \in \Omega, w \in \mathcal{W}, a \in A_{Z}^{-}}\left(a^{-\rho_{Q}}(1+\|\log a\|)^{-N}|f(\omega a w)|\right) .
$$

Moreover, due to the fact that $\mathbf{v}$ and $\mathbf{w}^{-1}$ are weight functions on $Z$, one gets that $G$ acts by left translations on $E_{N}$, and, for any compact subset $C$ of $G$, by changing $z$ into $z^{\prime}=g \cdot z$ in (2.5), one sees that:

There exists $c>0$ such that:

$$
p_{N}\left(L_{g} f\right) \leqslant c p_{N}(f), \quad g \in C, f \in E_{N} .
$$

But this action is not continuous. Let $V_{N}$ be the space of continuous vectors of $E_{N}$, i.e. the space of $f \in E_{N}$ such that the map $G \rightarrow E_{N}, g \mapsto L_{g} f$, is continuous. It is easy, using (2.7), to prove that $V_{N}$ is a closed $G$-invariant subspace of $E_{N}$ and $V_{N}$ is a continuous Banach representation of $G$.

\subsection{Lemma.}

(i) The space $V_{N}^{\infty}$ is equal to

$$
C_{\text {temp,N }}^{\infty}(Z):=\left\{f \in C^{\infty}(Z): p_{N, u}(f)<\infty, u \in U(\mathfrak{g})\right\},
$$

where $p_{N, u}(f)=p_{N}\left(L_{u} f\right)$.

(ii) The topology on $V_{N}^{\infty}$ is defined by the semi-norms $p_{N, u}, u \in U(\mathfrak{g})$. It is also defined by the semi-norms $p_{N, k}, k \in \mathbb{N}$ (cf. (2.2)), or $\Delta_{p_{N, 2 k}}, k \in \mathbb{N}$ (cf. (2.3)).

(iii) The topology on $V_{N}^{\infty}$ is defined by the semi-norms $q_{N, u}, u \in U(\mathfrak{g})$. It is also defined by the semi-norms $q_{N, k}, k \in \mathbb{N}$, or $\Delta_{q_{N, 2 k}}, k \in \mathbb{N}$.

Proof. Looking at the definition, it is easy to see that:

$$
V_{N}^{\infty} \subset C^{\infty}(Z)
$$

and is contained in $C_{\text {temp,N }}^{\infty}(Z)$. Reciprocally, let $f \in C_{\text {temp,N }}^{\infty}(Z)$. It is an element of $E_{N}$. Let us show that $f \in V_{N}$. This is a consequence of the mean value theorem:

If $X$ is in a compact neighborhood $B$ of 0 in $\mathfrak{g}, z \in Z$ and $t \in[0,1]$, then there exists $c_{t, X, z} \in[0,1]$ such that:

$$
\left(L_{\exp t X} f\right)(z)-f(z)=t\left(L_{X} f\right)\left(\exp \left(c_{t, X, z} X\right)^{-1} \cdot z\right) .
$$


Hence

$$
p_{N}\left(L_{\exp t X} f-f\right)=t \sup _{z \in Z}(1+\mathbf{w}(z))^{-N} \mathbf{v}(z)^{1 / 2}\left|\left(L_{X} f\right)\left(\exp \left(c_{t, X, z} X\right)^{-1} \cdot z\right)\right| .
$$

Changing $z$ into $\exp \left(c_{t, X, z} X\right)^{-1} \cdot z$ and using that $\mathbf{v}$ and $\mathbf{w}$ are weights (cf. (2.4)), one deduces easily that $f \in V_{N}$. To prove that $f \in V_{N}^{\infty}$, one can first show that the map $g \mapsto L_{g} f$ is 1-differentiable. It is clear that, if $X \in \mathfrak{g}$ and $g \in G, L_{X}\left(L_{g} f\right) \in C_{t e m p, N}^{\infty}(Z)$. Hence, by the previous discussion, one has $L_{X}\left(L_{g} f\right) \in V_{N}$. One can proceed similarly as above by studying:

$$
p_{N}\left(\frac{L_{\exp t X}\left(L_{g} f\right)-L_{g} f}{t}-L_{X}\left(L_{g} f\right)\right),
$$

using the Taylor expansion in 0 at order 2 of the function $t \mapsto L_{\exp t X}\left(L_{g} f\right)$. It implies that the map $g \mapsto L_{g} f$ has partial derivatives at order 1 given by $L_{X}\left(L_{g} f\right), X \in \mathfrak{g}$. Let us show that these partial derivatives are continuous from $G$ to $V_{N}$. First $g \mapsto L_{g} f$ is continuous by definition of $V_{N}$. Let $X_{1}, \ldots, X_{n}$ be a basis of $\mathfrak{g}$. Then, using that $L_{X}\left(L_{g} f\right)=L_{g}\left(L_{\operatorname{Ad}\left(g^{-1}\right) X} f\right)$, there exist real valued $C^{\infty}$-functions on $G, c_{i}, i=1, \ldots, n$, such that

$$
L_{X}\left(L_{g} f\right)=\sum_{i} c_{i}(g) L_{g}\left(L_{X_{i}} f\right) \text {. }
$$

But, as $f \in C_{t e m p, N}^{\infty}(Z), L_{X_{i}} f \in C_{\text {temp,N }}^{\infty}(Z)$ which has been seen to be contained in $V_{N}$. It follows that $g \mapsto L_{X}\left(L_{g} f\right)$ is continuous from $G$ to $V_{N}$. Thus, the map $g \mapsto L_{g} f$ is a $C^{1}$-map from $G$ to $V_{N}$. Then, using induction on the order of the partial derivatives, one shows that $g \mapsto L_{g} f$ has continuous partial derivatives at every order. Hence $f \in V_{N}^{\infty}$. This achieves to prove (i).

The point (ii) follows from [2, Proposition 3.5] and then (iii) follows from (2.6).

Let us define the notion of $Z$-tempered continuous $H$-fixed linear forms on a HarishChandra representation of $G, V^{\infty}$. If $V$ denotes the subspace of $K$-finite vectors of $V^{\infty}$, then a continuous $H$-fixed linear form $\eta$ is called $Z$-tempered if it satisfies:

There exists $N \in \mathbb{N}$ such that, for all $v \in V$ (resp. $\left.v \in V^{\infty}\right)$,

$$
m_{\eta, v} \in C_{t e m p, N}^{\infty}(Z)
$$

The first condition is the original definition of temperedness of [9, Definition 5.3 and Remark 5.4]. That this condition implies the second is proved in [11, Theorems 7.1 and 6.13(2)]. Denote by $\left(V^{-\infty}\right)_{\text {temp }}^{H}$ the space of $Z$-tempered continuous $H$-fixed linear forms on $V^{\infty}$.

2.3 Lemma. Let $f \in C^{\infty}(Z)$. The following conditions are equivalent:

(i) There exist a Harish-Chandra G-representation $V^{\infty}$, a Z-tempered contiuous linear form $\eta$ on $V^{\infty}$ and $v_{0} \in V^{\infty}$ such that $m_{\eta, v_{0}}=f$; 
(ii) There exist $N \in \mathbb{N}$ and a Harish-Chandra sub-representation $V_{1}^{\infty}$ of $C_{\text {temp,N }}^{\infty}(Z)$ such that $f \in V_{1}^{\infty}$.

We define $\mathcal{A}_{\text {temp }}(Z)$ as the set of $f \in C^{\infty}(Z)$ satisfying (one of) these equivalent conditions. If $N \in \mathbb{N}, \mathcal{A}_{\text {temp }, N}(Z)$ is the set of $f \in C^{\infty}(Z)$ satisfying (ii) for this precise $N$.

Proof. Let $f \in C^{\infty}(Z)$ satisfying (i). Then, from Lemma 2.2(i) and the definition of temperedness, $\left\{m_{\eta, v}: v \in V^{\infty}\right\}$ is a sub-representation of $C_{\text {temp }, N}^{\infty}(Z)$ for some $N \in \mathbb{N}$. Let $V$ be the underlying $(\mathfrak{g}, K)$-module of $V^{\infty}$ and let $V_{1}^{\infty}$ be the closure in $C_{\text {temp,N }}^{\infty}(Z)$ of $\left\{m_{\eta, v}: v \in V\right\}$. It is an SF-representation of $G$ (cf. Lemma 2.1(ii)). Let $\left(V_{1}^{\infty}\right)_{(K)}$ be the space of $K$-finite vectors in $V_{1}^{\infty}$. One has (cf. loc. cit.)

$$
\left(V_{1}^{\infty}\right)_{(K)}=\left\{m_{\eta, v}: v \in V\right\} .
$$

Hence $\left(V_{1}^{\infty}\right)_{(K)}$ is of finite length and $V_{1}^{\infty}$ is a Harish-Chandra representation of $G$. It is the SF-globalization of $\left\{m_{\eta, v}: v \in V\right\}$. Hence (cf. [16, Theorem 11.6.7]) there exists a surjective (because of (2.8)) continuous linear intertwining operator $T^{\prime}$ between $V^{\infty}$ and $V_{1}^{\infty}$ such that:

$$
T^{\prime}(v)=m_{\eta, v}, \quad v \in V .
$$

We claim that $T^{\prime}(v)=m_{\eta, v}$ for all $v \in V^{\infty}$. Let us show that, if a sequence $\left(v_{n}\right)$ in $V^{\infty}$ converges to $v,\left(m_{\eta, v_{n}}\right)$ converges to $m_{\eta, v}$ uniformly on compact sets. In fact, from (2.1), if $\Omega$ is a compact set in $G$, there exist a continuous semi-norm $q$ on $C^{\infty}(Z)$ and $N^{\prime} \in \mathbb{N}$ such that

$$
\left|<\eta, \pi\left(g^{-1}\right) v_{n}>-<\eta, \pi\left(g^{-1}\right) v>\right| \leqslant C q\left(v_{n}-v\right), \quad g \in \Omega H,
$$

for some $C>0$. Our claim follows.

From the fact that $\eta$ is a continuous $H$-fixed linear form on the SF-representation $V^{\infty}$, it is then easily seen that the map:

$$
T: v \mapsto m_{\eta, v}
$$

is a continuous map from $V^{\infty}$ into $C(Z)$. On the other hand, the embedding of $C_{\text {temp,N }}^{\infty}(Z)$ in $C(Z)$ is obviously continuous and linear. Then, by composition, the map $T^{\prime}$, given in (2.9), defines a continuous linear map from $V^{\infty}$ into $C(Z)$. Hence (2.9) implies by density that $T=T^{\prime}$. This implies that $T$ is a continuous and surjective linear map from $V^{\infty}$ to $V_{1}^{\infty}$. This shows that $m_{\eta, v_{0}} \in V_{1}^{\infty}$ and $V_{1}^{\infty}$ satisfies (ii).

Reciprocally, if $f$ satisfies (ii), let $\eta$ be the restriction to $V_{1}^{\infty}$ of the Dirac measure at $z_{0}$. Then $\left(V_{1}^{\infty}, \eta\right)$ satisfies (i) for $v_{0}=f$.

Let us remark that, for any $N_{1}, N_{2} \in \mathbb{N}$,

$$
N_{1} \leqslant N_{2} \text { implies } \mathcal{A}_{t e m p, N_{1}}(Z) \subset \mathcal{A}_{t e m p, N_{2}}(Z) .
$$

Indeed, this follows from the property:

$$
p_{N_{2}}(f) \leqslant p_{N_{1}}(f), \quad f \in C_{c}^{\infty}(Z),
$$

which implies that $C_{\text {temp,N }}^{\infty}(Z)$ is a subspace of $C_{\text {temp, } N_{2}}^{\infty}(Z)$. We endow $\mathcal{A}_{\text {temp,N }}(Z)$ with the topology induced by the topology of $C_{t e m p, N}^{\infty}(Z)$. 
2.4 Lemma. The space $\mathcal{A}_{\text {temp }}(Z)$ is a vector subspace of $C^{\infty}(Z)$.

Proof. As $\mathcal{A}_{\text {temp }}(Z)$ is the union $\bigcup_{N \in \mathbb{N}} \mathcal{A}_{\text {temp }, N}(Z)$ and according to (2.10), it is enough to prove that $\mathcal{A}_{\text {temp }, N}(Z)$ is a vector subspace of $C^{\infty}(Z)$. It is clear that if $f \in \mathcal{A}_{\text {temp,N }}(Z)$, one has $\lambda f \in \mathcal{A}_{\text {temp }, N}(Z)$ for $\lambda \in \mathbb{C}$. Let $f_{1}, f_{2} \in \mathcal{A}_{\text {temp }, N}(Z)$. For $i=1,2$, let $V_{i}^{\infty}$ be a Harish-Chandra sub-representation of $C_{t e m p, N}^{\infty}(Z)$ containing $f_{i}$. Let $V_{i}$ be the underlying $(\mathfrak{g}, K)$-module of $V_{i}^{\infty}$. Let $V=V_{1}+V_{2}$. It is a $(\mathfrak{g}, K)$-submodule of $C_{\text {temp,N }}^{\infty}(Z)_{(K)}$. Recall from Lemma 2.2 that $C_{t e m p, N}^{\infty}(Z)$ is the space of smooth vectors of a Banach representation. Then, from Lemma 2.1(ii), one sees that the closure of $V, V^{\infty}$, is a Harish-Chandra subrepresentation of $C_{\text {temp }, N}^{\infty}(Z)$ which contains $f_{1}+f_{2}$. Hence $f_{1}+f_{2} \in \mathcal{A}_{t e m p, N}(Z)$.

Recall that, if $V^{\infty}$ is a Harish-Chandra representation of $G$, then $\left(V^{-\infty}\right)^{H}$ is a finite dimensional vector space (cf. [12, Theorem 3.2]).

2.5 Lemma. Let $V^{\infty}$ be a Harish-Chandra representation of $G$. Then:

(i) The group $A_{Z, E}$ acts on the finite dimensional vector space $\left(V^{-\infty}\right)^{H}$.

(ii) If $\eta \in\left(V^{-\infty}\right)_{\text {temp }}^{H}$ and $a_{0} \in A_{Z, E}$, then $a_{0} \eta \in\left(V^{-\infty}\right)_{\text {temp }}^{H}$.

(iii) If $\eta \in\left(V^{-\infty}\right)_{\text {temp }}^{H}, \eta \neq 0$, transforms by a character $\chi$ under $A_{Z, E}$, then one has $|\chi(a)|=a^{\rho_{Q}}, a \in A_{Z, E}$.

(iv) If $\eta \in\left(V^{-\infty}\right)_{\text {temp }}^{H}$ and $v \in V^{\infty}$,

$$
a \longmapsto a^{-\rho_{Q}}<a \eta, v>
$$

is an exponential polynomial on $A_{Z, E}$ with unitary characters and polynomials having bounded degrees by the dimension of $\left(V^{-\infty}\right)^{H}$.

Proof. The assertion (i) follows from the fact that $\mathfrak{h}$ is normalized by $A_{Z, E}$ (cf. [11, equation (3.2)]) Let us look at $\left\langle\omega a w a_{0} \eta, v>\right.$, where $v \in V^{\infty}, \omega \in \Omega, w \in \mathcal{W}, a_{0} \in A_{Z, E}$ and $a \in A_{Z}$. Then, from [11, Lemma 3.5], as $\eta$ is $H$-fixed, this is equal to $\left\langle\omega a a_{0} w \eta, v\right\rangle$. Then, by using (2.6) and $\left\|\log a a_{0}\right\| \leqslant\|\log a\|+\left\|\log a_{0}\right\|$, one gets that $a_{0} \eta$ is $Z$-tempered. This shows (ii).

Let us now assume that $\eta$ transforms by a character $\chi$ under $A_{Z, E}$. As $\eta$ is $Z$-tempered,

$$
\left|a^{-\rho_{Q}}<a \eta, v>\right| \leqslant C(1+\|\log a\|)^{n}, \quad a \in A_{Z, E} .
$$

As $\langle a \eta, v\rangle=\chi(a)\langle\eta, v\rangle$, one then gets, assuming $v$ such that $\langle\eta, v\rangle \neq 0$, that $\left|\chi(a) a^{-\rho_{Q}}\right|=1$ for $a \in A_{Z, E}$ and hence (iii).

Let us prove (iv). As $A_{Z, E}$ acts on the finite dimensional vector space $\left(V^{-\infty}\right)^{H}$, it follows that, for all $v \in V^{\infty}$, the function on $A_{Z, E}, a \mapsto<a \eta, v>$, is an exponential polynomial function follows from the fact that $A_{Z, E}$ acts on the finite dimensional vector space $\left(V^{-\infty}\right)_{t e m p}^{H}$. If a character $\chi$ appears in the decomposition of this $A_{Z, E}$-module, there is a non zero $\eta_{\chi} \in$ $\left(V^{-\infty}\right)_{\text {temp }}^{H}$ which transforms by $\chi$ under $A_{Z, E}$. One concludes from (iii) that $a \mapsto a^{-\rho_{Q}} \chi(a)$ is unitary. Moreover the degrees of the polynomials are bounded by the dimension of the $A_{Z, E^{-}}$module $\left(V^{-\infty}\right)_{\text {temp }}^{H}$. 


\section{Differential equation for some functions on $Z$ wave- front and unimodular}

\subsection{Boundary degenerations of $Z$}

Let $I$ be a subset of $S$ and set:

$$
\begin{aligned}
\mathfrak{a}_{I} & =\left\{X \in \mathfrak{a}_{Z}: \alpha(X)=0, \alpha \in I\right\}, \\
\mathfrak{a}_{I}^{--} & =\left\{X \in \mathfrak{a}_{I}: \alpha(X)<0, \alpha \in S \backslash I\right\}, \\
A_{I} & =\exp \mathfrak{a}_{I} \subset A_{Z}, \\
A_{I}^{--} & =\exp \left(\mathfrak{a}_{I}^{--}\right) .
\end{aligned}
$$

Then there exists an algebraic Lie subalgebra $\mathfrak{h}_{I}$ of $\mathfrak{g}$ such that, for all $X \in \mathfrak{a}_{I}^{--}$, one has:

$$
\mathfrak{h}_{I}=\lim _{t \rightarrow+\infty} e^{\operatorname{ad} t X} \mathfrak{h}
$$

in the Grassmanian of $\mathfrak{g}$ (cf. [11, equation (3.6)]).

Let $H_{I}$ be the connected subgroup of $G$ corresponding to $\mathfrak{h}_{I}$ which is closed, as $\mathfrak{h}_{I}$ is algebraic. Let $Z_{I}=G / H_{I}$. Then $Z_{I}$ is a real spherical space for which:

(i) $P H_{I}$ is open,

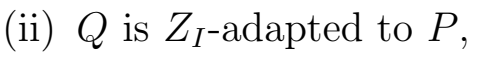

(iii) $\mathfrak{a}_{Z_{I}}=\mathfrak{a}_{Z}$ and $\mathfrak{a}_{Z_{I}}^{-}=\left\{X \in \mathfrak{a}_{Z}: \alpha(X) \leqslant 0, \alpha \in I\right\}$ contains $\mathfrak{a}_{Z}^{-}$

(cf. [11, Proposition 3.2]). Let $A_{Z_{I}}^{-}=\exp \mathfrak{a}_{Z_{I}}^{-}$. Similarly to $Z$, the real spherical space $Z_{I}$ has a polar decomposition:

$$
Z_{I}=\Omega_{I} A_{Z_{I}}^{-} \mathcal{W}_{I} \cdot z_{0, I}
$$

where $z_{0, I}=H_{I}, \Omega_{I}=\mathcal{F}_{I} K$, and $\mathcal{F}_{I}$ and $\mathcal{W}_{I}$ are finite sets in $G$ (cf. [11, Section 3.4.1]). Using Lemma 1.1, we can make the same kind of choice for $\mathcal{W}_{I}$ as for $\mathcal{W}$.

If $X \in \mathfrak{a}_{I}^{--}$, we define

$$
\beta_{I}(X)=\max _{\alpha \in S \backslash I} \alpha(X)<0
$$

and, if $a \in A_{I}^{--}$with $a=\exp X$, we set $a^{\beta_{I}}=e^{\beta_{I}(X)}$.

\subsection{Some estimates}

3.1 Lemma. Let $Y \in \mathfrak{h}_{I}$ and $N \in \mathbb{N}$. There exists a continuous semi-norm on $C_{\text {temp,N }}^{\infty}(Z)$, $p$, such that

$$
\left|\left(L_{Y} f\right)(a)\right| \leqslant p(f) a^{\rho_{Q}+\beta_{I}}(1+\|\log a\|)^{N}, \quad a \in A_{I}^{--}, f \in C_{t e m p, N}^{\infty}(Z) .
$$


Proof. If $Y \in \mathfrak{l} \cap \mathfrak{h}$,

$$
\left(L_{Y} f\right)(a)=0, \quad a \in A_{I} .
$$

Hence the conclusion of the Lemma holds for $Y \in \mathfrak{l} \cap \mathfrak{h}$.

Let $\alpha$ be a root of $\mathfrak{a}$ in $\mathfrak{u}$, i.e. $\alpha \in \Sigma_{\mathfrak{u}}$, and let $X_{-\alpha} \in \mathfrak{g}^{-\alpha}$. We have defined (cf. (1.4)) $X_{\alpha, \beta} \in \mathfrak{g}^{\beta}$ for $\alpha \in \Sigma_{\mathfrak{u}}, \beta \in \Sigma_{\mathfrak{u}}$ and $X_{\alpha, 0} \in(\mathfrak{l} \cap \mathfrak{h})^{\perp_{\mathfrak{l}}}$, where $(\mathfrak{l} \cap \mathfrak{h})^{\perp_{\mathfrak{l}}}$ is the orthogonal in $\mathfrak{l}$ of $\mathfrak{l} \cap \mathfrak{h}$ for the scalar product on $\mathfrak{g}$ (cf. Section 1) restricted to $\mathfrak{l}$. We set (cf. [11, beginning of Section 3.3]):

$$
X_{\alpha, \beta}^{I}= \begin{cases}X_{\alpha, \beta}, & \text { if } \alpha+\beta \in\langle I\rangle, \\ 0, & \text { otherwise }\end{cases}
$$

where $\langle I\rangle \subset \mathbb{N}_{0}[S]$ is the monoid generated by $I$, and we define (cf. loc.cit. equation (3.7)):

$$
T_{I}\left(X_{-\alpha}\right)=\sum_{\beta \in \Sigma_{\mathfrak{u}} \cup\{0\}} X_{\alpha, \beta}^{I} .
$$

Then (cf. loc.cit. equation (3.9)):

$$
Y_{-\alpha}=X_{-\alpha}+T_{I}\left(X_{-\alpha}\right) \in \mathfrak{h}_{I}
$$

and $\mathfrak{l} \cap \mathfrak{h}$ and the $Y_{-\alpha}$, when $\alpha$ and $X_{-\alpha}$ vary, generate $\mathfrak{h}_{I}$.

Let $\tilde{a}=s(a)(\operatorname{cf} .(1.3)$ for the definition of $s$ ). Then let us show that:

$$
\operatorname{Ad}(\tilde{a}) Y_{-\alpha}=\tilde{a}^{-\alpha} Y_{-\alpha}
$$

One has $\operatorname{Ad}(\tilde{a}) X_{-\alpha}=\tilde{a}^{-\alpha} X_{-\alpha}$ and $\operatorname{Ad}(\tilde{a}) X_{\alpha, \beta}=\tilde{a}^{\beta} X_{\alpha, \beta}$. But $\alpha+\beta \in I$. Hence $\tilde{a}^{\alpha+\beta}=1$, as $a \in A_{I}$. Our claim follows.

Let us study $\left(L_{Y_{-\alpha}} f\right)(a)$ for $a \in A_{I}^{--}$and $f \in \mathcal{A}_{t e m p, N}(Z)$. One has:

$$
\begin{aligned}
\left(L_{Y_{-\alpha}} f\right)(a) & =\left(L_{\tilde{a}^{-1}}\left(L_{Y_{-\alpha}} f\right)\right)\left(z_{0}\right) \\
& =\tilde{a}^{\alpha}\left(L_{Y_{-\alpha}} L_{\tilde{a}^{-1}} f\right)\left(z_{0}\right) .
\end{aligned}
$$

Let us notice that:

$$
Y_{-\alpha}+\sum_{\beta \in \Sigma_{\mathfrak{u}} \cup\{0\}, \alpha+\beta \notin\langle I\rangle} X_{\alpha, \beta} \in \mathfrak{h} .
$$

Hence one has:

$$
\begin{aligned}
\left(L_{Y_{-\alpha}} f\right)(a) & =-\tilde{a}^{\alpha} \sum_{\beta \in \Sigma_{\mathfrak{u}} \cup\{0\}, \alpha+\beta \notin\langle I\rangle}\left(L_{X_{\alpha, \beta}} L_{\tilde{a}^{-1}} f\right)\left(z_{0}\right) \\
& =-\sum_{\beta \in \Sigma_{\mathfrak{u}} \cup\{0\}, \alpha+\beta \notin\langle I\rangle} \tilde{a}^{\alpha+\beta}\left(L_{\tilde{a}^{-1}} L_{X_{\alpha, \beta}} f\right)\left(z_{0}\right) .
\end{aligned}
$$

But $\tilde{a}^{\alpha+\beta}=a^{\alpha+\beta}$ as $a \in A_{I} \subset A_{Z}$ and $\alpha+\beta \in S$. Then, as $\left(L_{\tilde{a}^{-1}} L_{X_{\alpha, \beta}} f\right)\left(z_{0}\right)=L_{X_{\alpha, \beta}} f(a)$, one has:

$$
\left(L_{Y_{-\alpha}} f\right)(a)=-\sum_{\beta \in \Sigma_{\mathfrak{u}} \cup\{0\}, \alpha+\beta \notin\langle I\rangle} a^{\alpha+\beta}\left(L_{X_{\alpha, \beta}} f\right)(a) .
$$

If $\alpha+\beta \notin\langle I\rangle$ as above and $L_{X_{\alpha, \beta}} f \neq 0$, one has $\alpha+\beta \in \mathcal{M} \backslash\langle I\rangle$ and, from the definition of $\beta_{I}(\mathrm{cf} .(3.1))$ :

$$
a^{\alpha+\beta} \leqslant a^{\beta_{I}}, \quad a \in A_{I}^{--} .
$$


Then

$$
\left|\left(L_{Y_{-\alpha}} f\right)(a)\right| \leqslant a^{\beta_{I}} \sum_{\beta \in \Sigma_{\mathfrak{u}} \cup\{0\}, \alpha+\beta \notin\langle I\rangle}\left|\left(L_{X_{\alpha, \beta}} f\right)(a)\right| .
$$

Hence we get the inequality of the Lemma for $Y=Y_{-\alpha}$ by taking

$$
p=\sum_{\beta \in \Sigma_{\mathfrak{u}} \cup\{0\}, \alpha+\beta \notin\langle I\rangle} p_{X_{\alpha, \beta}, N} .
$$

Let us recall (cf. e.g. [11, Section 5.1]) that $Z$ is said wave-front if

$$
\mathfrak{a}_{Z}^{-}=\left(\mathfrak{a}^{-}+\mathfrak{a}_{H}\right) / \mathfrak{a}_{H} .
$$

We will now make the following hypothesis on $Z$ :

\section{Let us assume from now, unless specified, that $Z$ is wave-front and unimodular.}

Let $I \subset S$. Let $F_{Q}$ be the subset of the set of simple roots $\Pi$ of $\mathfrak{a}$ in $\mathfrak{n}$, such that $Q$ is the parabolic subgroup of $G$ corresponding to the roots $\Sigma_{\mathfrak{n}} \backslash\left\langle F_{Q}\right\rangle$. Let us recall some results of [11, Corollary 5.6]. As $Z$ is wave-front, there exists a minimal set $F_{I} \subset \Pi$ which contains $F_{Q}$ and such that:

$$
\left\langle F_{I}\right\rangle \cap \mathbb{N}_{0}[S]=\langle I\rangle .
$$

Moreover, if $Q_{I}$ denotes the parabolic subgroup of $G$ containing $Q$ and corresponding to the roots $\Sigma_{\mathfrak{n}} \backslash\left\langle F_{I}\right\rangle$, and $Q_{I}=L_{I} U_{I}$ is its Levi decomposition with $A \subset L_{I}$, one has:

$$
\left(L_{I} \cap H\right)_{0} U_{I}^{-} \subset H_{I} \subset Q_{I}^{-},
$$

where $Q_{I}^{-}$is the parabolic subgroup of $G$ opposite to $Q_{I}$ containing $A$. Let us denote by $\mathfrak{u}_{I}^{-}$ the nilradical of the parabolic subalgebra $\mathfrak{q}_{I}^{-}$.

3.2 Lemma. Let $X \in \mathfrak{u}_{I}^{-}$and $u \in U(\mathfrak{g})$. There exists a continuous semi-norm on $C_{\text {temp }, N}^{\infty}(Z)$, $q$, such that, for all $f \in C_{\text {temp }, N}^{\infty}(Z)$,

$$
\begin{array}{r}
\left|\left(L_{X} L_{u} f\right)\left(a_{Z} a_{I}\right)\right| \leqslant q(f)\left(a_{Z} a_{I}\right)^{\rho_{Q}} a_{I}^{\beta_{I}}\left(1+\left\|\log a_{Z}\right\|\right)^{N}\left(1+\left\|\log a_{I}\right\|\right)^{N} \\
a_{Z} \in A_{Z}^{-}, a_{I} \in A_{I}^{--} .
\end{array}
$$

Proof. As $L_{u}$ is a continuous operator on $C_{t e m p, N}^{\infty}(Z)$, it is enough to prove the Lemma for $u=1$. By linearity, we can assume that $X=X_{-\alpha}$ is a weight vector in $\mathfrak{a}$ for the weight $-\alpha$, where $\alpha$ is a root of $\mathfrak{a}$ in $\mathfrak{u}_{I}$.

As $X_{-\alpha} \in \mathfrak{h}_{I}, T_{I}\left(X_{-\alpha}\right)=0$ and $Y_{-\alpha}=X_{-\alpha}$. In particular, $\operatorname{Ad}(\tilde{a}) Y_{-\alpha}=\tilde{a}^{-\alpha} Y_{-\alpha}$ for $a \in A_{Z}$ (recall that in the proof of Lemma 3.1, this is true only for $a \in A_{I}$ ). Hence (3.2) is true for $a \in A_{Z}$ and:

$$
\left(L_{Y_{-\alpha}} f\right)(a)=\sum_{\beta \in \Sigma_{\mathfrak{u}} \cup\{0\}, \alpha+\beta \notin\langle I\rangle} a^{\alpha+\beta}\left(L_{X_{\alpha, \beta}} f\right)(a), \quad a \in A_{Z} .
$$


Let us assume $a=a_{Z} a_{I}$ with $a_{Z} \in A_{Z}^{-}, a_{I} \in A_{I}^{--}$. Then, as $a_{Z}^{\alpha+\beta} \leqslant 1$, and as $a_{I} \in A_{I}^{--}$, $a_{I}^{\alpha+\beta} \leqslant a_{I}^{\beta_{I}}$, by definition of $\beta_{I}$ (cf. (3.1)), one gets $a^{\alpha+\beta} \leqslant a_{I}^{\beta_{I}}$. Moreover, as elements of $U(\mathfrak{g})$ act continuously on $C_{\text {temp,N }}^{\infty}(Z)$, there exists a continuous semi-norm $p$ on $C_{\text {temp }, N}^{\infty}(Z)$ such that, for all $\beta \in \Sigma_{\mathfrak{u}} \cup\{0\}$,

$$
\left|\left(L_{X_{\alpha, \beta}} f\right)\left(a_{Z} a_{I}\right)\right| \leqslant p(f)\left(a_{Z} a_{I}\right)^{\rho_{Q}}\left(1+\left\|\log a_{Z}\right\|\right)^{N}\left(1+\left\|\log a_{I}\right\|\right)^{N}, \quad f \in C_{\text {temp }, N}^{\infty}(Z) .
$$

To get this inequality, we have used that:

$$
\left\|\log \left(a_{Z} a_{I}\right)\right\| \leqslant\left\|\log a_{Z}\right\|+\left\|\log a_{I}\right\| .
$$

The Lemma follows.

\subsection{Algebraic preliminaries}

Let $A_{L_{I}}$ be the maximal vector subgroup of the center of the Levi subgroup $L_{I}$ of $Q_{I}$ contained in $A$. Then (cf. [11])

$$
\mathfrak{a}_{L_{I}} / \mathfrak{a}_{L_{I}} \cap \mathfrak{a}_{H} \simeq \mathfrak{a}_{I} \subset \mathfrak{a}_{Z}
$$

Let $\mathfrak{c}_{\mathfrak{l}_{I}}$ be the center of $\mathfrak{l}_{I}$ and ${ }^{0} \mathfrak{l}_{I}=\left[\mathfrak{l}_{I}, \mathfrak{l}_{I}\right]+\mathfrak{c}_{\mathfrak{l}_{I}} \cap \mathfrak{k}$. One has:

$$
\mathfrak{l}_{I}={ }^{0} \mathfrak{l}_{I} \oplus \mathfrak{a}_{L_{I}}
$$

Let $p r_{I}$ be the projection of $\mathfrak{l}_{I}$ on $\mathfrak{a}_{L_{I}}$ parallel to ${ }^{0} \mathfrak{l}_{I}$. Let $\rho_{Q_{I}}$ denote the half sum of the roots in $\Sigma^{+} \backslash\left\langle F_{I}\right\rangle$, i.e. the roots of $\mathfrak{a}$ in $\mathfrak{u}_{I}$. From [11, equation (3.9)] and the fact that $\mathfrak{a}_{L_{I}} \subset \mathfrak{a}$, one has $\mathfrak{a}_{L_{I}} \cap \mathfrak{h}_{I}=\mathfrak{a}_{L_{I}} \cap \mathfrak{h}$. Let us show that:

$$
\rho_{Q_{I}} \text { is trivial on } \mathfrak{a}_{L_{I}} \cap \mathfrak{h}_{I} \text {. }
$$

From [11, Lemma 3.11], $Z_{I}$ is also unimodular and, as $\mathfrak{l}_{I} \cap \mathfrak{h}_{I^{-}}$-modules,

$$
\mathfrak{g} / \mathfrak{h}_{I}=\mathfrak{u}_{I} \oplus\left(\mathfrak{l}_{I} / \mathfrak{l}_{I} \cap \mathfrak{h}_{I}\right) .
$$

In fact, the action of $\mathfrak{a}_{L_{I}} \cap \mathfrak{h}_{I}$ on $\mathfrak{l}_{I} / \mathfrak{l}_{I} \cap \mathfrak{h}_{I}$ is trivial. Hence the action of $\mathfrak{a}_{L_{I}} \cap \mathfrak{h}_{I}$ on $\mathfrak{u}_{I}$ has to be unimodular. Our claim follows. Let us define a function $d_{Q_{I}}$ on $L_{I}$ by:

$$
d_{Q_{I}}(l)=\left(\operatorname{det}\left(\operatorname{Ad} l_{\mid \mathfrak{u}_{I}}\right)\right)^{1 / 2}, \quad l \in L_{I} .
$$

In particular

$$
d_{Q_{I}}(a)=a^{\rho_{Q_{I}}}, \quad a \in A_{L_{I}} .
$$

Let us notice that, from (3.4),

$$
d_{Q_{I}} \text { is trivial on } A_{L_{I}} \cap A_{H} .
$$

We define an automorphism of $U\left(\mathfrak{l}_{I}\right)$ :

$$
\sigma_{I}: U\left(\mathfrak{l}_{I}\right) \rightarrow U\left(\mathfrak{l}_{I}\right)
$$


such that:

$$
L_{\sigma_{I}(X)}=d_{Q_{I}}^{-1} \circ L_{X} \circ d_{Q_{I}}, \quad X \in \mathfrak{l}_{I},
$$

i.e. $\sigma_{I}(X)=X-\rho_{Q_{I}}\left(p r_{I}(X)\right), X \in \mathfrak{l}_{I}$.

We define also a map $\mu_{I}: Z(\mathfrak{g}) \rightarrow Z\left(\mathfrak{l}_{I}\right)$ characterized by:

$$
z-\mu_{I}(z) \in \mathfrak{u}_{I}^{-} U(\mathfrak{g}), \quad z \in Z(\mathfrak{g}) .
$$

Then $\gamma_{I}:=\sigma_{I} \circ \mu_{I}: Z(\mathfrak{g}) \rightarrow Z\left(\mathfrak{l}_{I}\right)$ is the so-called Harish-Chandra homomorphism and one has:

$$
L_{\gamma_{I}(z)}=d_{Q_{I}}^{-1} \circ L_{\mu_{I}(z)} \circ d_{Q_{I}}, \quad z \in Z(\mathfrak{g}) .
$$

One knows that $Z\left(\mathfrak{l}_{I}\right)$ is a free module of finite rank over $\gamma_{I}(Z(\mathfrak{g}))$. Hence there exists a finite dimensional vector subspace $W$ of $Z\left(\mathfrak{l}_{I}\right)$ containing 1 such that the map:

$$
\begin{aligned}
\gamma_{I}(Z(\mathfrak{g})) \otimes W & \longrightarrow Z\left(\mathfrak{l}_{I}\right) \\
u \otimes v & \longmapsto u v
\end{aligned}
$$

is a linear bijection.

Let $\mathcal{I}$ be a finite codimensional ideal of $Z(\mathfrak{g})$ and let $\mathcal{J}=\gamma_{I}(\mathcal{I})$. Let $V$ be a finite dimensional vector subspace of $\gamma_{I}(Z(\mathfrak{g}))$ containing 1 such that $\gamma_{I}(Z(\mathfrak{g}))=\mathcal{J} \oplus V$. Hence:

$$
\begin{aligned}
Z\left(\mathfrak{l}_{I}\right) & =(\mathcal{J} \oplus V) W \\
& =\mathcal{J} W \oplus V W
\end{aligned}
$$

where $\mathcal{J} W$ (resp. $V W$ ) is the linear span of $\{u v: u \in \mathcal{J}, v \in W\}(\operatorname{resp} .\{u v: u \in V, v \in W\})$.

We set $W_{\mathcal{I}}:=V W$. Let us notice that:

$$
\mathcal{J} W=\mathcal{J} \gamma_{I}(Z(\mathfrak{g})) W=\mathcal{J} Z\left(\mathfrak{l}_{I}\right)
$$

We see that, if $\mathcal{I}$ is the kernel of a character $\chi$ of $Z(\mathfrak{g})$, one may and will take $V=\mathbb{C} 1$, hence $W_{\mathcal{I}}=W$. One has:

$$
Z\left(\mathfrak{l}_{I}\right)=W_{\mathcal{I}} \oplus \mathcal{J} W .
$$

Let $s_{\mathcal{I}}$, resp. $q_{\mathcal{I}}$, be the linear map from $Z\left(\mathfrak{l}_{I}\right)$ to $W_{\mathcal{I}}$, resp. $\mathcal{J} W$, deduced from this direct sum decomposition. The algebra $Z\left(\mathfrak{l}_{I}\right)$ acts on $W_{\mathcal{I}}$ by a representation $\rho_{\mathcal{I}}$ defined by:

$$
\rho_{\mathcal{I}}(u) v=s_{\mathcal{I}}(u v), \quad u \in Z\left(\mathfrak{l}_{I}\right), v \in W_{\mathcal{I}} .
$$

In fact:

The representation $\left(\rho_{\mathcal{I}}, W_{\mathcal{I}}\right)$ is isomorphic to the natural representation of $Z\left(\mathfrak{l}_{I}\right)$ on $Z\left(\mathfrak{l}_{I}\right) / Z\left(\mathfrak{l}_{I}\right) \mathcal{J}$.

We notice that:

$$
u v=\rho_{\mathcal{I}}(u) v+q_{\mathcal{I}}(u v) .
$$


Let $\left(v_{i}\right)_{i=1, \ldots, n}$ be a basis of $W$. Then:

$$
q_{\mathcal{I}}(u v)=\sum_{i=1}^{n} \gamma_{I}\left(z_{i}(u, v, \mathcal{I})\right) v_{i}
$$

where the $z_{i}(u, v, \mathcal{I})$ are in $\mathcal{I}$. Let us recall that:

$$
\gamma_{I}\left(z_{i}(u, v, \mathcal{I})\right)=d_{Q_{I}}^{-1} \circ L_{\mu_{I}\left(z_{i}(u, v, \mathcal{I})\right)} \circ d_{Q_{I}}
$$

and that:

$$
\mu_{I}\left(z_{i}(u, v, \mathcal{I})\right) \in z_{i}(u, v, \mathcal{I})+\mathfrak{u}_{I}^{-} U(\mathfrak{g}) .
$$

Let us take a basis $\left(u_{I, j}^{-}\right)_{j=1, \ldots, p}$ of $\mathfrak{u}_{I}^{-}$. We may assume that each $u_{I, j}^{-}$is a weight vector for $\mathfrak{a}$ with weight $\alpha_{j}$. Then

$$
\mu_{I}\left(z_{i}(u, v, \mathcal{I})\right)=z_{i}(u, v, \mathcal{I})+\sum_{j=1}^{p} u_{I, j}^{-} v_{i, j}(u, v, \mathcal{I})
$$

where $v_{i, j}(u, v, \mathcal{I}) \in U(\mathfrak{g})$.

Let $\mathfrak{j}_{\mathbb{C}}$ be a complex Cartan subalgebra of $\mathfrak{g}_{\mathbb{C}}$ of the form $\mathfrak{t}_{\mathbb{C}} \oplus \mathfrak{a}_{\mathbb{C}}$, where $\mathfrak{t}$ is a maximal abelian subalgebra of $\mathfrak{m}$, the centralizer of $\mathfrak{a}$ in $\mathfrak{k}$. Let $W\left(\mathfrak{g}_{\mathbb{C}}, \mathfrak{j}_{\mathbb{C}}\right)$ be the corresponding Weyl group.

One has $\mathfrak{a}=\mathfrak{a}_{L_{I}} \oplus\left(\mathfrak{a} \cap{ }^{0} \mathfrak{l}_{I}\right)$. Hence one has natural inclusions:

$$
\mathfrak{a}_{L_{I}}^{*} \subset \mathfrak{a}^{*} \text { and } \mathfrak{a}_{\mathbb{C}}^{*} \subset \mathfrak{j}_{\mathbb{C}}^{*}
$$

If $\Lambda \in \mathfrak{j}_{\mathbb{C}}^{*}$, let $\chi_{\Lambda}=\chi_{\lambda}^{\mathfrak{g}}$ be the character of $Z(\mathfrak{g})$ corresponding to $\Lambda$ via the Harish-Chandra isomorphism $\gamma$ from $Z(\mathfrak{g})$ onto $S\left(\mathfrak{j}_{\mathbb{C}}\right)^{W\left(\mathfrak{g}_{\mathbb{C}}, \mathfrak{j}_{\mathbb{C}}\right)}$. More precisely,

$$
\chi_{\Lambda}(u)=(\gamma(u))(\Lambda), \quad u \in Z(\mathfrak{g}) .
$$

We define similarly the character $\chi_{\Lambda}^{\mathfrak{l}_{I}}$ of $Z\left(\mathfrak{l}_{I}\right)$.

When $\mathcal{I}=\mathcal{I}_{\Lambda}:=\operatorname{Ker} \chi_{\Lambda}$, we take, as we have already said, $W_{\mathcal{I}}=W$ and we write $s_{\Lambda}$ instead of $s_{\mathcal{I}}, q_{\Lambda}$ instead of $q_{\mathcal{I}}, \rho_{\Lambda}$ instead of $\rho_{\mathcal{I}}$ and $(u, v, \Lambda)$ instead of $(u, v, \mathcal{I})$. Let us show that, for $u \in Z\left(\mathfrak{l}_{I}\right), s_{\Lambda}(u)$ and $q_{\Lambda}(u)$ are polynomial in $\Lambda$. It is enough to prove this for $u=\gamma_{I}(z) v$ where $z \in Z(\mathfrak{g})$ and $v \in W$. Then $u=\left(\gamma_{I}(z)-\chi_{\Lambda}(z)\right) v+\chi_{\Lambda}(z) v$. Hence $q_{\Lambda}(u)=\left(\gamma_{I}(z)-\chi_{\Lambda}(z)\right) v \in Z\left(\mathfrak{l}_{I}\right) \mathcal{J}$ and $s_{\Lambda}(u)=\chi_{\Lambda}(z) v \in W$. Our claim follows. It implies easily that:

$$
z_{i}(u, v, \Lambda) \text { in (3.7) depends polynomially on } \Lambda \text {. }
$$

This implies, as $\mu_{I}$ is linear, that:

$$
v_{i, j}(u, v, \Lambda) \text { in }(3.9) \text { depends polynomially on } \Lambda \text {. }
$$

Using Harish-Chandra isomorphisms, one sees that:

Each simple subquotient of the representation $\rho_{\Lambda}$ of $Z\left(\mathfrak{l}_{I}\right)$ is given by some character of the form $\chi_{\mu}^{\mathfrak{l}_{I}}$, where $\mu$ varies in $W\left(\mathfrak{g}_{\mathbb{C}}, \mathfrak{j}_{\mathbb{C}}\right) \Lambda$.

Let us notice that $\chi_{\mu}^{\mathfrak{l}_{I}}=\chi_{w \mu}^{\mathfrak{l}_{I}}$, where $w \in W\left(\mathfrak{l}_{I, \mathbb{C}}, \mathfrak{j}_{\mathbb{C}}\right)$. 


\subsection{The function $\varphi_{f}$ on $L_{I}$ and related differential equations}

If $\mathcal{I}$ is a cofinite dimensional ideal in $Z(\mathfrak{g})$ and $N \in \mathbb{N}^{*}$, we denote by $\mathcal{A}_{\text {temp,N }}(Z: \mathcal{I})$ $\left(\right.$ resp. $\left.\mathcal{A}_{\text {temp }}(Z: \mathcal{I})\right)$ the space of $f \in \mathcal{A}_{\text {temp }, N}(Z)$ (resp. $\left.\mathcal{A}_{\text {temp }}(Z)\right)$ annihilated by $\mathcal{I}$.

Let $f \in \mathcal{A}_{\text {temp }, N}(Z: \mathcal{I})$ that we might view as a function on $G$. We denote by $\varphi_{f}$ the function on $L_{I}$ with values in $W_{\mathcal{I}}^{*}$ defined by:

$$
<\varphi_{f}(l), v>=\left(L_{v}\left(d_{Q_{I}}^{-1} f\right)\right)(l), \quad l \in L_{I}, v \in W_{\mathcal{I}} .
$$

This is a function on $L_{I}$, not necessarily on $L_{I} / L_{I} \cap H$.

Let us study $L_{u} \varphi_{f}$ for $u \in Z\left(\mathfrak{l}_{I}\right)$. For $v \in W_{\mathcal{I}}$,

$$
<L_{u} \varphi_{f}(l), v>=L_{u v} d_{Q_{I}}^{-1} f(l), \quad l \in L_{I} .
$$

Using (3.6) and (3.7), we get:

$$
<L_{u} \varphi_{f}(l), v>=L_{\rho_{\mathcal{I}}(u) v} d_{Q_{I}}^{-1} f(l)+\sum_{i=1}^{n} d_{Q_{I}}^{-1}\left(d_{Q_{I}} L_{\gamma_{I}\left(z_{i}(u, v, \mathcal{I})\right) v_{i}} d_{Q_{I}}^{-1} f\right)(l), \quad l \in L_{I} .
$$

From (3.8) and (3.9), we then deduce:

$$
\begin{aligned}
<L_{u} \varphi_{f}(l), v>= & L_{\rho_{\mathcal{I}}(u) v} d_{Q_{I}}^{-1} f(l)+\sum_{i=1}^{n} d_{Q_{I}}^{-1}\left(L_{z_{i}(u, v, \mathcal{I})} d_{Q_{I}} L_{v_{i}} d_{Q_{I}}^{-1} f\right)(l) \\
& +\sum_{i=1}^{n} \sum_{j=1}^{p} d_{Q_{I}}^{-1}\left(L_{u_{I, j}^{-}} L_{v_{i, j}(u, v, \mathcal{I})} d_{Q_{I}} L_{v_{i}} d_{Q_{I}}^{-1} f\right)(l), \quad l \in L_{I} .
\end{aligned}
$$

One has

$$
d_{Q_{I}} \circ L_{v_{i}} \circ d_{Q_{I}}^{-1}=L_{v_{i}^{I}}
$$

for an element $v_{i}^{I}$ of $Z\left(\mathfrak{l}_{I}\right)$. The operators $L_{z_{i}(u, v, \mathcal{I})}$ and $L_{v_{i}^{I}}$ commute. Hence, as $z_{i}(u, v, \mathcal{I}) \in \mathcal{I}$ and $f \in \mathcal{A}_{\text {temp }, N}(Z: \mathcal{I})$, one has:

$$
L_{z_{i}(u, v, \mathcal{I})} L_{v_{i}^{I}} f=0 .
$$

Let us define a function on $L_{I}$ with values in $W_{\mathcal{I}}^{*}, \psi_{f, u}$, by:

$$
<\psi_{f, u}(l), v>=-\sum_{i, j} d_{Q_{I}}^{-1} L_{u_{I, j}^{-}} L_{v_{i, j}^{\prime}(u, v, \mathcal{I})} f(l), \quad v \in W_{\mathcal{I}}, l \in L_{I},
$$

where $v_{i, j}^{\prime}(u, v, \mathcal{I})=v_{i, j}(u, v, \mathcal{I}) v_{i}^{I}$. From (3.14) and (3.15), we deduce:

$$
L_{u} \varphi_{f}={ }^{t} \rho_{\mathcal{I}}(u) \varphi_{f}-\psi_{f, u}, \quad u \in Z\left(\mathfrak{l}_{I}\right) .
$$

Let $X \in \mathfrak{a}_{L_{I}} \cap \mathfrak{a}_{H}$. As $f$ restricted to $L_{I}$ and $d_{Q_{I}}$ are left invariant by $\exp X$ (cf. (3.5) for $d_{Q_{I}}$, , one sees that:

$$
L_{X} \varphi_{f}=0
$$




\subsection{The function $\Phi_{f}$ on $A_{Z}$ and related differential equations}

Let us consider the natural projection $p: \mathfrak{a}_{L} \rightarrow \mathfrak{a}_{Z}=\mathfrak{a}_{L} / \mathfrak{a}_{H}$ and let $s$ be the section of $p$ introduced in (1.3). From [11, Corollary 5.6], we have that $\mathfrak{a}_{I}$ is equal to the projection of $\mathfrak{a}_{L_{I}}$ on $\mathfrak{a}_{Z}$. Hence:

One may and will choose the section $s$ such that $s\left(\mathfrak{a}_{I}\right) \subset \mathfrak{a}_{L_{I}}$.

Recall that the map $s$ is be also denoted by $X \mapsto \tilde{X}$ or $a \mapsto \tilde{a}$ for the corresponding morphism of Lie groups.

Recall that $A_{Z}=A_{Z_{I}}$. Let $\rho_{L_{I} \cap Q}$ be the half sum of the roots of $\mathfrak{a}$ in $\mathfrak{l}_{I} \cap \mathfrak{u}$. In particular $\rho_{Q}=\rho_{L_{I} \cap Q}+\rho_{Q_{I}}$ on $\mathfrak{a}$.

Let $f \in \mathcal{A}_{\text {temp }, N}(Z: \mathcal{I})$ and let us define a function $\Phi_{f}: A_{Z} \rightarrow W_{\mathcal{I}}^{*}$ by:

$$
\Phi_{f}\left(a_{Z}\right)=\tilde{a}_{Z}^{-\rho_{L_{I} \cap Q}} \varphi_{f}\left(\tilde{a}_{Z}\right), \quad a_{Z} \in A_{Z} .
$$

Let us recall (cf. (3.13)) that, for $v \in W_{\mathcal{I}}$,

$$
<\varphi_{f}(\tilde{a}), v>=L_{v}\left(d_{Q_{I}}^{-1} f\right)\left(\tilde{a} \cdot z_{0}\right)=d_{Q_{I}}^{-1}\left(L_{v^{I}} f\right)\left(\tilde{a} \cdot z_{0}\right), \quad \tilde{a} \in A_{L_{I}},
$$

where $v^{I}=d_{Q_{I}} \circ v \circ d_{Q_{I}}^{-1} \in Z\left(\mathfrak{l}_{I}\right)$. Hence, for $a_{Z} \in A_{Z}$ and $v \in W_{\mathcal{I}}$,

$$
<\Phi_{f}\left(a_{Z}\right), v>=\tilde{a}_{Z}^{-\rho_{L_{I}} \cap Q} d_{Q_{I}}^{-1}\left(\tilde{a}_{Z}\right)\left(L_{v^{I}} f\right)\left(\tilde{a}_{Z} \cdot z_{0}\right) .
$$

One has:

$$
\begin{aligned}
\tilde{a}_{Z}^{-\rho_{L_{I} \cap Q}} d_{Q_{I}}^{-1}\left(\tilde{a}_{Z}\right) & =\tilde{a}_{Z}^{-\rho_{L_{I} \cap Q}} \tilde{a}_{Z}^{-\rho_{Q_{I}}} \\
& =\tilde{a}_{Z}^{-\rho_{Q}} .
\end{aligned}
$$

Moreover $\rho_{Q}$ is trivial on $\mathfrak{a}_{H}$ (cf. (3.4)). Hence $\tilde{a}_{Z}^{-\rho_{Q}}=a_{Z}^{-\rho_{Q}}$. But $\tilde{a}_{Z} \cdot z_{0}=a_{Z}$. This leads to:

$$
<\Phi_{f}\left(a_{Z}\right), v>=a_{Z}^{-\rho_{Q}}\left(L_{v^{I}} f\right)\left(a_{Z}\right), \quad v \in W_{\mathcal{I}}, a_{Z} \in A_{Z}
$$

This shows that $\Phi_{f}$ does not depend on the section $s$.

Let us study $L_{X} \Phi_{f}$ for $X \in \mathfrak{a}_{I}$. It is equal to $L_{\tilde{X}} \Phi_{f}$, where $\tilde{X} \in \mathfrak{a}_{L_{I}}$. If $a_{I} \in A_{I}$, $\tilde{a}_{I}^{-\rho_{L_{I} \cap Q}}=1$ as $\tilde{a}_{I} \in A_{L_{I}}$ by our choice of the section $s$. Now we use (3.18) and (3.17) to get:

$$
L_{\tilde{X}} \Phi_{f}\left(a_{Z}\right)={ }^{t} \rho_{\mathcal{I}}(\tilde{X}) \Phi_{f}\left(a_{Z}\right)-\tilde{a}_{Z}^{-\rho_{L_{I} \cap Q}} \psi_{f, \tilde{X}}\left(a_{Z}\right), \quad a_{Z} \in A_{Z}
$$

Let us study $\tilde{a}_{Z}^{-\rho_{L_{I} \cap Q}} \psi_{f, \tilde{X}}\left(a_{Z}\right)$ using (3.16):

$$
<\tilde{a}_{Z}^{-\rho_{L_{I} \cap Q}} \psi_{f, \tilde{X}}\left(\tilde{a}_{Z}\right), v>=-\tilde{a}_{Z}^{-\rho_{L_{I} \cap Q}} d_{Q_{I}}^{-1}\left(\tilde{a}_{Z}\right) \sum_{i, j}\left(L_{u_{I, j}^{-} v_{i, j}^{\prime}(\tilde{X}, v, \mathcal{I})} f\right)\left(a_{Z}\right), \quad a_{Z} \in A_{Z}, v \in W_{\mathcal{I}} .
$$

Using (3.19), one has:

$$
<\tilde{a}_{Z}^{-\rho_{L_{I} \cap Q}} \psi_{f, \tilde{X}}\left(\tilde{a}_{Z}\right), v>=<\Psi_{f, X}\left(a_{Z}\right), v>, \quad a_{Z} \in A_{Z}, v \in W_{\mathcal{I}},
$$


where $\Psi_{f, X}: A_{Z} \rightarrow W_{\mathcal{I}}^{*}$ is defined by:

$$
<\Psi_{f, X}\left(a_{Z}\right), v>=-a_{Z}^{-\rho_{Q}} \sum_{i, j}\left(L_{u_{I, j}^{-}} L_{v_{i, j}^{\prime}(\tilde{X}, v, \mathcal{I})} f\right)\left(a_{Z}\right), \quad v \in W_{\mathcal{I}}, X \in \mathfrak{a}_{I}, a_{Z} \in A_{Z} .
$$

Using (3.21) and (3.22), one gets:

$$
L_{X} \Phi_{f}={ }^{t} \rho_{\mathcal{I}}(\tilde{X}) \Phi_{f}-\Psi_{f, X}, \quad X \in \mathfrak{a}_{I} .
$$

One sets:

$$
\Gamma_{\mathcal{I}}(X)=-{ }^{t} \rho_{\mathcal{I}}(\tilde{X}), \quad X \in \mathfrak{a}_{I}
$$

Hence, one has the important relation:

$$
L_{X} \Phi_{f}=-\Gamma_{\mathcal{I}}(X) \Phi_{f}-\Psi_{f, X}, \quad X \in \mathfrak{a}_{I}
$$

We notice that $\Gamma_{\mathcal{I}}$ is a representation of the abelian Lie algebra $\mathfrak{a}_{I}$ on $W_{\mathcal{I}}^{*}$. For $\lambda \in \mathfrak{a}_{I, \mathbb{C}}^{*}$, one denotes by $W_{\mathcal{I}, \lambda}^{*}$ the space of joint generalized eigenvectors of $W_{\mathcal{I}}^{*}$ by the endomorphisms $\Gamma_{\mathcal{I}}(X), X \in \mathfrak{a}_{I}$, for the eigenvalue $\lambda$. Let $\mathcal{Q}_{\mathcal{I}}$ be the (finite) subset of $\mathfrak{a}_{I, \mathbb{C}}^{*}$ such that $W_{\mathcal{I}, \lambda}^{*} \neq$ $\{0\}$. One has:

$$
W_{\mathcal{I}}^{*}=\bigoplus_{\lambda \in \mathcal{Q}_{\mathcal{I}}} W_{\mathcal{I}, \lambda}^{*} .
$$

If $\lambda \in \mathcal{Q}_{\mathcal{I}}$, let $E_{\lambda}$ be the projector of $W_{\mathcal{I}}^{*}$ onto $W_{\mathcal{I}, \lambda}^{*}$ parallel to the sum of the other $W_{\mathcal{I}, \mu}^{*}$ 's. We endow $W_{\mathcal{I}}^{*}$ with a scalar product and if $T \in \operatorname{End}\left(W_{\mathcal{I}}^{*}\right)$, we denote by $\|T\|$ its Hilbert-Schmidt norm. It is clear that $E_{\lambda}$ commutes with the operators $\Gamma_{\mathcal{I}}(X), X \in \mathfrak{a}_{I}$. We set

$$
\Phi_{f, \lambda}=E_{\lambda} \Phi_{f}
$$

The proofs of the following results (Lemma 3.3 up to Proposition 3.14) follow closely the work of Harish-Chandra (cf. [5, Section 22]). Here $M_{1}^{+}$is replaced by $A_{Z}^{-}$and $M_{1}$ by $A_{Z_{I}}^{-}$.

3.3 Lemma. One has, for all $a_{Z} \in A_{Z}, T \in \mathbb{R}, X_{I} \in \mathfrak{a}_{I}, \lambda \in \mathcal{Q}_{\mathcal{I}}$,

$$
\Phi_{f}\left(a_{Z} \exp \left(T X_{I}\right)\right)=e^{T \Gamma_{\mathcal{I}}\left(X_{I}\right)} \Phi_{f}\left(a_{Z}\right)+\int_{0}^{T} e^{(T-t) \Gamma_{\mathcal{I}}\left(X_{I}\right)} \Psi_{f, X_{I}}\left(a_{Z} \exp \left(t X_{I}\right)\right) d t
$$

$$
\Phi_{f, \lambda}\left(a_{Z} \exp \left(T X_{I}\right)\right)=e^{T \Gamma_{\mathcal{I}}\left(X_{I}\right)} \Phi_{f, \lambda}\left(a_{Z}\right)+\int_{0}^{T} E_{\lambda} e^{(T-t) \Gamma_{\mathcal{I}}\left(X_{I}\right)} \Psi_{f, X_{I}}\left(a_{Z} \exp \left(t X_{I}\right)\right) d t .
$$

Proof. The equality (i) is an immediate consequence of (3.25). Indeed, we apply the elementary result on first order linear differential equation to the function $t \mapsto F(t)=$ $\Phi_{f}\left(a_{Z} \exp \left(t X_{I}\right)\right)$, whose derivative is $F^{\prime}(t)=-L_{X_{I}} \Phi_{f}\left(a_{Z} \exp \left(t X_{I}\right)\right)$ satisfies

$$
F^{\prime}(t)=\Gamma_{\mathcal{I}}\left(X_{I}\right) F(t)+\Psi_{f, X_{I}}\left(a_{Z} \exp \left(t X_{I}\right)\right) .
$$

The equality (ii) follows by applying $E_{\lambda}$ to both sides of the equality of (i). 
Let

$$
E_{\lambda}(X):=E_{\lambda} \exp \left(\Gamma_{\mathcal{I}}(X)-\lambda(X)\right)
$$

for $X \in \mathfrak{a}_{I}$. Since $E_{\lambda}\left(\Gamma_{\mathcal{I}}(X)-\lambda(X)\right)$ is nilpotent, one has:

3.4 Lemma. We can choose $c \geqslant 0$ such that:

$$
\left\|E_{\lambda}(X)\right\| \leqslant c(1+\|X\|)^{N_{\mathcal{I}}}, \quad X \in \mathfrak{a}_{I}
$$

where $N_{\mathcal{I}}$ is the dimension of $W_{\mathcal{I}}$.

The function $\Psi_{f, X}$ is a function on $A_{Z}$ and one is interested in its derivatives along $Y \in \mathfrak{a}_{Z}$. On one hand, one has:

$$
L_{Y} a_{Z}^{-\rho_{Q}}=\rho_{Q}(Y) a_{Z}^{-\rho_{Q}}
$$

One the other hand, one has:

$$
\tilde{Y} u_{I, j}^{-}=\left[\tilde{Y}, u_{I, j}^{-}\right]+u_{I, j}^{-} \tilde{Y}=\alpha_{j}(\tilde{Y}) u_{I, j}^{-}+u_{I, j}^{-} \tilde{Y}
$$

Hence $L_{Y} \Psi_{f, X}$ and more generally $L_{u} \Psi_{f, X}, u \in S\left(\mathfrak{a}_{Z}\right)$, is a function of the same type than $\Psi_{f, X}(\operatorname{see}(3.23))$.

3.5 Lemma. Fix $u \in S\left(\mathfrak{a}_{Z}\right)$.

(i) There exists a continuous semi-norm on $C_{\text {temp }, N}^{\infty}(Z), p_{u}$, such that:

$$
\begin{aligned}
\left\|L_{u} \Phi_{f}\left(a_{Z} \exp X_{I}\right)\right\| \leqslant p_{u}(f)\left(1+\left\|\log a_{Z}\right\|\right)^{N}\left(1+\left\|X_{I}\right\|\right)^{N}, \\
\\
a_{Z} \in A_{Z}^{-}, X_{I} \in \mathfrak{a}_{I}^{-}, f \in \mathcal{A}_{t e m p, N}(Z: \mathcal{I}) .
\end{aligned}
$$

(ii) There exists a continuous semi-norm on $C_{\text {temp,N }}^{\infty}(Z), q_{u}$, such that:

$$
\begin{array}{r}
\left\|L_{u} \Psi_{f, X}\left(a_{Z} \exp X_{I}\right)\right\| \leqslant q_{u}(f) e^{\beta_{I}\left(X_{I}\right)}\left(1+\left\|\log a_{Z}\right\|\right)^{N}\left(1+\left\|X_{I}\right\|\right)^{N} \\
a_{Z} \in A_{Z}^{-}, X_{I} \in \mathfrak{a}_{I}^{--}, f \in \mathcal{A}_{\text {temp }, N}(Z: \mathcal{I}) .
\end{array}
$$

Proof. Let us first prove (i). It is easy to see, using (3.20), that:

$$
<L_{u} \Phi_{f}\left(a_{Z}\right), v>=a_{Z}^{-\rho_{Q}}\left(L_{v^{I}} L_{u^{\prime}} f\right)\left(a_{Z}\right), \quad v \in W_{\mathcal{I}}
$$

for some $u^{\prime} \in S\left(\mathfrak{a}_{Z}\right)$ with $\operatorname{deg} u^{\prime} \leqslant \operatorname{deg} u$. Then (i) follows from the continuity of the operator $L_{v^{I}} L_{u^{\prime}}$ on $C_{\text {temp,N }}^{\infty}(Z)$ and the definition of $C_{t e m p, N}^{\infty}(Z)$ (cf. Lemma 2.2(i)). By definition of $\Psi_{f, X}$ (cf. (3.23)), one gets (ii) using Lemma 3.2.

We say that an integral depending on a parameter converges uniformly if the absolute value of the integrand is bounded by an integrable function independently of the parameter. 
3.6 Lemma. Let us fix $u \in S\left(a_{Z}\right), \lambda \in \mathcal{Q}_{\mathcal{I}}, X_{I} \in \mathfrak{a}_{I}^{--}$, and let us suppose that $\operatorname{Re} \lambda\left(X_{I}\right)>$ $\beta_{I}\left(X_{I}\right)$. Then

(i) The integral

$$
\int_{0}^{\infty} E_{\lambda} e^{-t \Gamma_{\mathcal{I}}\left(X_{I}\right)} L_{u} \Psi_{f, X_{I}}\left(a_{Z_{I}} \exp \left(t X_{I}\right)\right) d t
$$

converges uniformly on any compact subset of $A_{Z_{I}}^{-}$.

(ii) The map

$$
a_{Z_{I}} \mapsto \int_{0}^{\infty} E_{\lambda} e^{-t \Gamma_{\mathcal{I}}\left(X_{I}\right)} \Psi_{f, X_{I}}\left(a_{Z_{I}} \exp \left(t X_{I}\right)\right) d t
$$

is a well-defined map on $A_{Z_{I}}^{-}$. Its derivatives along $u \in S\left(\mathfrak{a}_{Z}\right)$ are given by derivation under the integral sign.

Proof. One has

$$
E_{\lambda} e^{-t \Gamma_{\mathcal{I}}\left(X_{I}\right)}=e^{-t \lambda\left(X_{I}\right)} E_{\lambda} e^{t\left(\lambda\left(X_{I}\right)-\Gamma_{\mathcal{I}}\left(X_{I}\right)\right)}=e^{-t \lambda\left(X_{I}\right)} E_{\lambda}\left(-t X_{I}\right) .
$$

Hence, from Lemma 3.4, one has:

$$
\left\|E_{\lambda} e^{-t \Gamma_{\mathcal{I}}\left(X_{I}\right)}\right\| \leqslant c\left(1+\left\|t X_{I}\right\|\right)^{N_{\mathcal{I}}} e^{-t \operatorname{Re} \lambda\left(X_{I}\right)} .
$$

Using Lemma 3.5(ii) and (3.26), one can show that the integral in (i) converges uniformly for $a_{Z_{I}} \in A_{Z}^{-}$. Let $a_{Z_{I}}$ be in a compact subset $C$ of $A_{Z_{I}}^{-}$. There exists $T_{0}>0$ such that, for all $z \in$ $C, z \exp \left(T_{0} X_{I}\right) \in A_{Z}^{-}$. Writing $\int_{0}^{+\infty}=\int_{0}^{T_{0}}+\int_{T_{0}}^{+\infty}, a_{Z_{I}} \exp \left(t X_{I}\right)=a_{Z_{I}} \exp T_{0} X_{I} \exp \left(t-T_{0}\right) X_{I}$, and, using the uniform convergence proved above, one gets (i).

The assertion (ii) follows from (i) and the theorem on derivatives of integrals depending of a parameter.

Fix $f \in \mathcal{A}_{\text {temp }, N}(Z: \mathcal{I})$ and $\lambda \in \mathcal{Q}_{\mathcal{I}}$ and put, for $X_{I}$ as in Lemma 3.6, i.e. $\operatorname{Re} \lambda\left(X_{I}\right)>$ $\beta_{I}\left(X_{I}\right)$ :

$$
\begin{aligned}
\Phi & =\Phi_{f} \\
\Phi_{\lambda} & =\Phi_{f, \lambda} \\
\Psi_{X_{I}} & =\Psi_{f, X_{I}}, \\
\Phi_{\lambda, \infty}\left(a_{Z_{I}}, X_{I}\right) & =\lim _{T \rightarrow+\infty} e^{-T \Gamma_{\mathcal{I}}\left(X_{I}\right)} \Phi_{f, \lambda}\left(a_{Z_{I}} \exp \left(T X_{I}\right)\right), \quad a_{Z_{I}} \in A_{Z_{I}}^{-}
\end{aligned}
$$

It follows from Lemmas 3.3(ii) and 3.6 that this limit exists and is $C^{\infty}$ on $A_{Z_{I}}^{-}$. Moreover

$$
\begin{array}{r}
L_{u} \Phi_{\lambda, \infty}\left(a_{Z_{I}}, X_{I}\right)=L_{u} \Phi_{\lambda}\left(a_{Z_{I}}\right)+\int_{0}^{\infty} E_{\lambda} e^{-t \Gamma_{\mathcal{I}}\left(X_{I}\right)} L_{u} \Psi_{X_{I}}\left(a_{Z} \exp \left(t X_{I}\right)\right) d t \\
u \in S\left(\mathfrak{a}_{Z}\right), a_{Z_{I}} \in A_{Z_{I}}^{-}
\end{array}
$$


3.7 Lemma. For $X_{I} \in \mathfrak{a}_{I}^{--}$such that $\operatorname{Re} \lambda\left(X_{I}\right)>0$, one has:

$$
\Phi_{\lambda, \infty}\left(a_{Z_{I}}, X_{I}\right)=0, \quad a_{Z_{I}} \in A_{Z_{I}}^{-} .
$$

Proof. One has

$$
\left\|e^{-T \Gamma_{\mathcal{I}}\left(X_{I}\right)} \Phi_{\lambda}\left(a_{Z_{I}} \exp \left(T X_{I}\right)\right)\right\| \leqslant e^{-T \operatorname{Re} \lambda\left(X_{I}\right)}\left\|E_{\lambda}\left(-T X_{I}\right)\right\|\left\|\Phi\left(a_{Z_{I}} \exp \left(T X_{I}\right)\right)\right\|
$$

and, from Lemmas 3.4 and 3.5(i), the right hand side of the inequality tends to zero as $T \rightarrow+\infty$. Hence the Lemma follows from the definition (3.27) of $\Phi_{\lambda, \infty}\left(a_{Z_{I}}, X_{I}\right)$.

3.8 Lemma. Let $X_{1}, X_{2} \in \mathfrak{a}_{I}^{--}$and suppose that

$$
\operatorname{Re} \lambda\left(X_{i}\right)>\beta_{I}\left(X_{i}\right), \quad i=1,2 .
$$

Then

$$
\Phi_{\lambda, \infty}\left(a_{Z_{I}}, X_{1}\right)=\Phi_{\lambda, \infty}\left(a_{Z_{I}}, X_{2}\right), \quad a_{Z_{I}} \in A_{Z_{I}}^{-} .
$$

Proof. Same as the proof of [5, Lemma 22.8]. We give it for sake of completeness. Let $a_{Z_{I}} \in A_{Z_{I}}^{-}$. Applying Lemma 3.3(ii) to $X_{2}$ instead of $X_{I}$ and $T_{2}$ instead of $T$, one gets:

$$
\begin{aligned}
& e^{-\Gamma_{\mathcal{I}}\left(T_{1} X_{1}+T_{2} X_{2}\right)} \Phi_{\lambda}\left(a_{Z_{I}} \exp \left(T_{1} X_{1}\right) \exp \left(T_{2} X_{2}\right)\right) \\
= & e^{-T_{1} \Gamma_{\mathcal{I}}\left(X_{1}\right)} \Phi_{\lambda}\left(a_{Z_{I}} \exp \left(T_{1} X_{1}\right)\right) \\
& +\int_{0}^{T_{2}} E_{\lambda} e^{-\Gamma_{\mathcal{I}}\left(T_{1} X_{1}-t_{2} X_{2}\right)} \Psi_{X_{2}}\left(a_{Z_{I}} \exp \left(T_{1} X_{1}+t_{2} X_{2}\right)\right) d t_{2},
\end{aligned}
$$

for $T_{1}, T_{2}>0$. From Lemmas 3.4 and 3.5(ii) applied to $T_{1} X_{1}+t_{2} X_{2}$ instead of $X_{I}$, one sees that:

$$
\int_{0}^{\infty}\left\|E_{\lambda} e^{-\Gamma_{\mathcal{I}}\left(T_{1} X_{1}-t_{2} X_{2}\right)}\right\|\left\|\Psi_{X_{2}}\left(a_{Z_{I}} \exp \left(T_{1} X_{1}+t_{2} X_{2}\right)\right)\right\| d t_{2}
$$

tends to 0 when $T_{1} \rightarrow+\infty$. Hence:

$$
\begin{aligned}
& \lim _{T_{1}, T_{2} \rightarrow+\infty} e^{-\Gamma_{\mathcal{I}}\left(T_{1} X_{1}+T_{2} X_{2}\right)} \Phi_{\lambda}\left(a_{Z_{I}} \exp \left(T_{1} X_{1}+T_{2} X_{2}\right)\right) \\
= & \lim _{T_{1} \rightarrow+\infty} e^{-\Gamma_{\mathcal{I}}\left(T_{1} X_{1}\right)} \Phi_{\lambda}\left(a_{Z_{I}} \exp \left(T_{1} X_{1}\right)\right) \\
= & \Phi_{\lambda, \infty}\left(a_{Z_{I}}, X_{1}\right) .
\end{aligned}
$$

Since the left side is symmetrical in $X_{1}$ and $X_{2}$, one then deduces that:

$$
\Phi_{\lambda, \infty}\left(a_{Z_{I}}, X_{1}\right)=\Phi_{\lambda, \infty}\left(a_{Z_{I}}, X_{2}\right) .
$$


We decompose $\mathcal{Q}_{\mathcal{I}}$ into three disjoints subsets $\mathcal{Q}_{\mathcal{I}}^{+}, \mathcal{Q}_{\mathcal{I}}^{0}$ and $\mathcal{Q}_{\mathcal{I}}^{-}$as follows:

(1) $\lambda \in \mathcal{Q}_{\mathcal{I}}^{+}$if $\operatorname{Re} \lambda(X)>0$ for some $X \in \mathfrak{a}_{I}^{--}$,

(2) $\lambda \in \mathcal{Q}_{\mathcal{I}}^{0}$ if $\operatorname{Re} \lambda(X)=0$ for all $X \in \mathfrak{a}_{I}^{--}$,

(3) $\lambda \in \mathcal{Q}_{\mathcal{I}}^{-}$if $\lambda \notin \mathcal{Q}_{\mathcal{I}}^{+} \cup \mathcal{Q}_{\mathcal{I}}^{0}$, i.e. for all $X \in \mathfrak{a}_{I}^{--}, \operatorname{Re} \lambda(X) \leqslant 0$ and there exists $X \in \mathfrak{a}_{I}^{--}$such that $\operatorname{Re} \lambda(X)<0$.

3.9 Lemma. Fix $\lambda \in \mathcal{Q}_{\mathcal{I}}^{+}$and suppose that $X \in \mathfrak{a}_{I}^{--}$is such that $\operatorname{Re} \lambda(X)>\beta_{I}(X)$. Then, for any $a_{Z_{I}} \in A_{Z_{I}}^{-}$,

$$
\Phi_{\lambda, \infty}\left(a_{Z_{I}}, X\right)=0
$$

and, for any $u \in S\left(\mathfrak{a}_{Z}\right)$,

$$
L_{u} \Phi_{\lambda}\left(a_{Z_{I}} \exp (T X)\right)=-\int_{T}^{\infty} E_{\lambda} e^{-(t-T) \Gamma_{\mathcal{I}}(X)} L_{u} \Psi_{X}\left(a_{Z_{I}} \exp (t X)\right) d t, \quad T \in \mathbb{R} .
$$

Proof. Since $\lambda \in \mathcal{Q}_{\mathcal{I}}^{+}$, there exists $X_{0} \in \mathfrak{a}_{I}^{--}$such that $\operatorname{Re} \lambda\left(X_{0}\right)>0$. Then, from Lemma 3.7, $\Phi_{\lambda, \infty}\left(a_{Z_{I}}, X_{0}\right)=0$, and, from Lemma 3.8, as $\operatorname{Re} \lambda\left(X_{0}\right)>0>\beta_{I}\left(X_{0}\right)$, one has $\Phi_{\lambda, \infty}\left(a_{Z_{I}}, X\right)=$ $\Phi_{\lambda, \infty}\left(a_{Z_{I}}, X_{0}\right)$ for any $X \in \mathfrak{a}_{I}^{--}$such that $\operatorname{Re} \lambda(X)>\beta_{I}(X)$. This proves the first part of the Lemma. The second part follows from (3.28) by change of variables and when we replace $a_{Z_{I}}$ by $a_{Z_{I}} \exp (T X)$.

3.10 Corollary. Let $\lambda \in \mathcal{Q}_{\mathcal{I}}^{+}$. Suppose $X \in \mathfrak{a}_{I}^{--}$is such that $\operatorname{Re} \lambda(X) \geqslant \beta_{I}(X) / 2$. Then, for $u \in S\left(\mathfrak{a}_{Z}\right), a_{Z_{I}} \in A_{Z_{I}}^{-}$and $T \geqslant 0$,

$$
\left\|L_{u} \Phi_{\lambda}\left(a_{Z_{I}} \exp (T X)\right)\right\| \leqslant e^{T \beta_{I}(X) / 2} \int_{T}^{\infty} e^{-t \beta_{I}(X) / 2}\left\|E_{\lambda}((T-t) X)\right\|\left\|L_{u} \Psi_{X}\left(a_{Z_{I}} \exp (t X)\right)\right\| d t
$$

Proof. Since $\beta_{I}(X)<0$ and $\operatorname{Re} \lambda(X) \geqslant \beta_{I}(X) / 2$, one has in particular $\operatorname{Re} \lambda(X)>\beta_{I}(X)$. Then one can see from Lemmas 3.9 and 3.6 that:

$$
\left\|L_{u} \Phi_{\lambda}\left(a_{Z_{I}} \exp (T X)\right)\right\| \leqslant \int_{T}^{\infty} e^{-(t-T) \operatorname{Re} \lambda(X)}\left\|E_{\lambda}((T-t) X)\right\|\left\|L_{u} \Psi_{X}\left(a_{Z_{I}} \exp (t X)\right)\right\| d t .
$$

Our assertion follows, since $\operatorname{Re} \lambda(X) \geqslant \beta_{I}(X) / 2$ implies that $-(t-T) \operatorname{Re} \lambda(X) \leqslant-(t-$ T) $\beta_{I}(X) / 2$ for $t \geqslant T$.

3.11 Lemma. Suppose $\lambda \in \mathcal{Q}_{\mathcal{I}}$, and $X \in \mathfrak{a}_{I}^{--}$is such that $\operatorname{Re} \lambda(X) \leqslant \beta_{I}(X) / 2$. Then

$$
\begin{array}{r}
\left\|L_{u} \Phi_{\lambda}\left(a_{Z_{I}} \exp (T X)\right)\right\| \leqslant \\
\quad e^{T \beta_{I}(X) / 2\left(\left\|E_{\lambda}(T X)\right\|\left\|L_{u} \Phi\left(a_{Z_{I}}\right)\right\|\right.} \\
\left.\quad+\int_{0}^{\infty} e^{-t \beta_{I}(X) / 2}\left\|E_{\lambda}((T-t) X)\right\|\left\|L_{u} \Psi_{X}\left(a_{Z_{I}} \exp (t X)\right)\right\| d t\right), \\
T \geqslant 0, u \in S\left(\mathfrak{a}_{Z}\right), a_{Z_{I}} \in A_{Z_{I}}^{-} .
\end{array}
$$


Proof. We use Lemma 3.3(ii) and the inequality $(T-t) \operatorname{Re} \lambda(X) \leqslant(T-t) \beta_{I}(X) / 2$ for $t \leqslant T$ in order to get an analogue of the inequality of the Lemma where $\int_{0}^{\infty}$ is replaced by $\int_{0}^{T}$. The Lemma follows.

Like in [5, after the proof of Lemma 22.8], one sees that one can choose $0<\delta \leqslant 1 / 2$ such that:

$$
\operatorname{Re} \lambda(X) \leqslant \delta \beta_{I}(X), \quad X \in \mathfrak{a}_{I}^{--}, \lambda \in \mathcal{Q}_{\mathcal{I}}^{-} .
$$

3.12 Lemma. Let $\lambda \in \mathcal{Q}_{\mathcal{I}}^{-}$and $X \in \mathfrak{a}_{I}^{--}$. Then, for $u \in S\left(\mathfrak{a}_{Z}\right), a_{Z_{I}} \in A_{Z_{I}}^{-}, T \geqslant 0$,

$$
\begin{aligned}
\left\|L_{u} \Phi_{\lambda}\left(a_{Z_{I}} \exp (T X)\right)\right\| \leqslant & e^{T \delta \beta_{I}(X)}\left(\left\|E_{\lambda}(T X)\right\|\left\|L_{u} \Phi\left(a_{Z_{I}}\right)\right\|\right. \\
& \left.+\int_{0}^{\infty} e^{-t \beta_{I}(X) / 2}\left\|E_{\lambda}((T-t) X)\right\|\left\|L_{u} \Psi_{X}\left(a_{Z_{I}} \exp (t X)\right)\right\| d t\right) .
\end{aligned}
$$

Proof. This is proved like Lemma 3.11, using that $\operatorname{Re} \lambda(X) \leqslant \delta \beta_{I}(X)$ and $0<\delta \leqslant 1 / 2$.

Let $\lambda \in \mathcal{Q}_{\mathcal{I}}^{0}$. It follows from Lemma 3.8 and the definition of $\beta_{I}$ (cf. (3.1)) that:

$$
\text { For } a_{Z_{I}} \in A_{Z_{I}}^{-}, \Phi_{\lambda, \infty}\left(a_{Z_{I}}, X\right) \text { is independent of } X \in \mathfrak{a}_{I}^{--} \text {. }
$$

We will denote it by $\Phi_{\lambda, \infty}\left(a_{Z_{I}}\right)$.

3.13 Lemma. Let $\lambda \in \mathcal{Q}_{\mathcal{I}}^{0}$ and $X \in \mathfrak{a}_{I}^{--}$. Then one has, for $u \in S\left(\mathfrak{a}_{Z}\right), T \geqslant 0$ and $a_{Z_{I}} \in A_{Z_{I}}^{-}$,

$$
\begin{aligned}
& \left\|L_{u} \Phi_{\lambda}\left(a_{Z_{I}} \exp (T X)\right)-L_{u} \Phi_{\lambda, \infty}\left(a_{Z_{I}} \exp (T X)\right)\right\| \\
\leqslant & e^{T \beta_{I}(X) / 2} \int_{0}^{\infty} e^{-t \beta_{I}(X) / 2}\left\|E_{\lambda}((T-t) X)\right\|\left\|L_{u} \Psi_{X}\left(a_{Z_{I}} \exp (t X)\right)\right\| d t .
\end{aligned}
$$

Proof. From (3.28), one deduces

$$
L_{u} \Phi_{\lambda, \infty}\left(a_{Z_{I}} \exp (T X)\right)=L_{u} \Phi_{\lambda}\left(a_{Z_{I}} \exp (T X)\right)+\int_{T}^{\infty} E_{\lambda} e^{-(t-T) \Gamma_{\mathcal{I}}(X)} L_{u} \Psi_{X}\left(a_{Z_{I}} \exp (t X)\right) d t .
$$

The Lemma now follows from the fact that $(T-t) \beta_{I}(X) \geqslant 0$ if $t \geqslant T$.

We define now:

$$
\Phi_{\lambda, \infty}\left(a_{Z_{I}}\right)=0, \quad a_{Z_{I}} \in A_{Z_{I}}^{-}, \lambda \in \mathcal{Q}_{\mathcal{I}}^{+} \cup \mathcal{Q}_{\mathcal{I}}^{-} .
$$

3.14 Proposition. Let $\lambda \in \mathcal{Q}_{\mathcal{I}}, X \in \mathfrak{a}_{I}^{--}$and $u \in S\left(\mathfrak{a}_{Z}\right)$. Then, for $a_{Z_{I}} \in A_{Z_{I}}^{-}, T \geqslant 0$,

$$
\begin{aligned}
& \left\|L_{u} \Phi_{\lambda}\left(a_{Z_{I}} \exp (T X)\right)-L_{u} \Phi_{\lambda, \infty}\left(a_{Z_{I}} \exp (T X)\right)\right\| \\
\leqslant & e^{T \delta \beta_{I}(X)}\left(\left\|E_{\lambda}(T X)\right\|\left\|L_{u} \Phi\left(a_{Z_{I}}\right)\right\|\right. \\
& \left.+\int_{0}^{\infty} e^{-t \beta_{I}(X) / 2}\left\|E_{\lambda}((T-t) X)\right\|\left\|L_{u} \Psi_{X}\left(a_{Z_{I}} \exp (t X)\right)\right\| d t\right) .
\end{aligned}
$$

Proof. If $\lambda \in \mathcal{Q}_{\mathcal{I}}^{0} \cup \mathcal{Q}_{\mathcal{I}}^{-}$, our assertion follows from Lemmas 3.12 and 3.13. On the other hand, if $\lambda \in \mathcal{Q}_{\mathcal{I}}^{+}$, we can apply Lemmas 3.9 and 3.11, and Corollary 3.10. 


\section{Definition of the constant term and its properties}

Let us recall that $I$ is a subset of $S$ and $\mathcal{I}$ a finite codimensional ideal in $Z(\mathfrak{g})$.

\subsection{Some estimates}

In this Subsection, we establish some estimates analogous to the ones given in [5, Section 23].

4.1 Lemma. We fix a compact set $\mathcal{C}$ in $\mathfrak{a}_{I}^{--}$and choose $\varepsilon_{0}>0$ such that $\beta_{I}(X) \leqslant-2 \varepsilon_{0}$ for all $X \in \mathcal{C}$. We put $\varepsilon=\delta \varepsilon_{0}$, where $\delta$ is given by (3.29). Let $u \in S\left(\mathfrak{a}_{Z}\right)$. Then there exists a continuous semi-norm $q$ on $C_{\text {temp }, N}^{\infty}(Z)$ such that, for all $\lambda \in \mathcal{Q}_{\mathcal{I}}, T \geqslant 0, X \in \mathcal{C}, a_{Z} \in A_{Z}^{-}$ and $f \in \mathcal{A}_{\text {temp }, N}(Z: \mathcal{I})$,

$$
\left\|L_{u} \Phi_{f, \lambda}\left(a_{Z} \exp (T X)\right)-L_{u} \Phi_{f, \lambda, \infty}\left(a_{Z} \exp (T X)\right)\right\| \leqslant e^{-\varepsilon T} q(f)\left(1+\left\|\log a_{Z}\right\|\right)^{N} .
$$

Proof. As $A_{Z}^{-}$is contained in $A_{Z_{I}}^{-}$, this follows from Proposition 3.14, Lemmas 3.5(ii) and 3.4.

4.2 Lemma. Let $\lambda \in \mathcal{Q}_{\mathcal{I}}$. One has:

$$
\Phi_{f, \lambda, \infty}\left(a_{Z_{I}} \exp X\right)=e^{\Gamma_{\mathcal{I}}(X)} \Phi_{f, \lambda, \infty}\left(a_{Z_{I}}\right), \quad X \in \mathfrak{a}_{I}, a_{Z_{I}} \in A_{Z_{I}}^{-}, f \in \mathcal{A}_{t e m p, N}(Z: \mathcal{I}) .
$$

Proof. One may assume $\lambda \in \mathcal{Q}_{\mathcal{I}}^{0}$. From Lemma 3.3(ii) applied with $T=1$, one has, for $a_{Z} \in A_{Z}, X \in \mathfrak{a}_{I}$,

$$
e^{-\Gamma_{\mathcal{I}}(X)} \Phi_{\lambda}\left(a_{Z} \exp X\right)=\Phi_{\lambda}\left(a_{Z}\right)+\int_{0}^{1} E_{\lambda} e^{-t \Gamma_{\mathcal{I}}(X)} \Psi_{X}\left(a_{Z} \exp (t X)\right) d t
$$

Let $Y \in \mathfrak{a}_{I}^{--}$. Replacing $a_{Z}$ by $a_{Z_{I}} \exp (T Y)$, with $a_{Z_{I}} \in A_{Z_{I}}$, and multiplying by $e^{-T \Gamma_{\mathcal{I}}(Y)}$, one gets:

$$
\begin{aligned}
e^{-\Gamma_{\mathcal{I}}(X+T Y)} \Phi_{\lambda}\left(a_{Z_{I}} \exp (X+T Y)\right)= & e^{-\Gamma_{\mathcal{I}}(T Y)} \Phi_{\lambda}\left(a_{Z_{I}} \exp (T Y)\right) \\
& +\int_{0}^{1} E_{\lambda} e^{-\Gamma_{\mathcal{I}}(t X+T Y)} \Psi_{X}\left(a_{Z_{I}} \exp (t X+T Y)\right) d t
\end{aligned}
$$

One can choose $T_{0}>0$ such that $a_{Z_{I}} \exp \left(T_{0} Y\right) \in A_{Z}^{-}$. If $T$ is sufficiently large, $t X+(T-$ $\left.T_{0}\right) Y \in \mathfrak{a}_{I}^{--}$for all $t \in[0,1]$. Recalling that $\lambda \in \mathcal{Q}_{\mathcal{I}}^{0}$, it follows from Lemma 3.5(ii) applied to $a_{Z}=a_{Z_{I}} \exp \left(T_{0} Y\right)$ and $X_{I}=t X+\left(T-T_{0}\right) Y$ that, if $a_{Z_{I}} \in A_{Z_{I}}^{-}$, the integral in this equality tends to 0 as $T \rightarrow+\infty$. Recalling the definition of $\Phi_{f, \lambda, \infty}$ (cf. (3.27)), one gets

$$
e^{-\Gamma_{\mathcal{I}}(X)} \Phi_{f, \lambda, \infty}\left(a_{Z_{I}} \exp X\right)=\Phi_{f, \lambda, \infty}\left(a_{Z_{I}}\right), \quad X \in \mathfrak{a}_{I}, a_{Z_{I}} \in A_{Z_{I}}^{-}
$$


4.3 Lemma. Let $\lambda \in \mathcal{Q}_{\mathcal{I}}^{0}$. There exists a continuous semi-norm $p$ on $C_{\text {temp }, N}^{\infty}(Z: \mathcal{I})$ such that, for all $f \in \mathcal{A}_{\text {temp }, N}(Z: \mathcal{I})$,

$$
\left\|\Phi_{f, \lambda, \infty}\left(a_{Z_{I}}\right)\right\| \leqslant p(f)\left(1+\left\|\log a_{Z_{I}}\right\|\right)^{N+\operatorname{dim} W_{\mathcal{I}}}, \quad a_{Z_{I}} \in A_{Z_{I}}^{-} .
$$

Proof. We fix $X \in \mathfrak{a}_{I}^{--}$. Let $a_{Z_{I}} \in A_{Z_{I}}^{-}$. If $t$ is large enough, $a_{Z_{I}} \exp (t X) \in A_{Z}^{-}$. More precisely, if $a_{Z_{I}}=\exp Y$ with $Y \in \mathfrak{a}_{Z_{I}}, t$ has to be such that $\alpha(Y+t X) \leqslant 0$ for all $\alpha \in S \backslash I$. For this, it is enough that $t \geqslant\left|\frac{\alpha(Y)}{\alpha(X)}\right|$ for all $\alpha \in S \backslash I$. But $\left|\frac{\alpha(Y)}{\alpha(X)}\right|$ is bounded above by $C\|Y\|$ for some constant $C>0$. We will take:

$$
T=C\|Y\|
$$

and write $a_{Z_{I}}=a_{Z} \exp (-T X)$ with $a_{Z}=a_{Z_{I}} \exp (T X) \in A_{Z}^{-}$. One has, from Lemma 4.2,

$$
\Phi_{f, \lambda, \infty}\left(a_{Z} \exp (-T X)\right)=e^{-T \Gamma_{\mathcal{I}}(X)} \Phi_{f, \lambda, \infty}\left(a_{Z}\right) .
$$

As $\lambda \in \mathcal{Q}_{\mathcal{I}}^{0},\left\|E_{\lambda} e^{-T \Gamma_{\mathcal{I}}(X)}\right\|$ is bounded by a constant times $(1+T\|X\|)^{N_{\mathcal{I}}}$, where $N_{\mathcal{I}}$ is the dimension of $W_{\mathcal{I}}$ (cf. Lemma 3.4). Using (4.1) and as $X$ is fixed, one concludes that there exists $C_{1}>0$ such that:

$$
\left\|E_{\lambda} e^{-T \Gamma_{\mathcal{I}}(X)}\right\| \leqslant C_{1}\left(1+\left\|\log a_{Z_{I}}\right\|\right)^{N_{\mathcal{I}}} .
$$

We remark that $\left\|\log a_{Z}\right\| \leqslant\left\|\log a_{Z_{I}}\right\|+\|T X\|$ is bounded by some constant times $\left\|\log a_{Z_{I}}\right\|$ because $T=C\|Y\|$ and $\|X\|$ is fixed. Then, using (4.2), the Lemma follows from Lemma 4.1 for $T=0$ and Lemma 3.5(i) for $X_{I}=0$.

Let $\mathcal{I}$ be a finite codimensional ideal in $Z(\mathfrak{g}), I \subset S, N \in \mathbb{N}^{*}$ and $f \in \mathcal{A}_{\text {temp }, N}(Z: \mathcal{I})$. Let us define

$$
\tilde{f}_{I}\left(a_{Z_{I}}\right):=\sum_{\lambda \in \mathcal{Q}_{I}^{0}}<\Phi_{f, \lambda, \infty}\left(a_{Z_{I}}\right), 1>, \quad a_{Z_{I}} \in A_{Z_{I}}^{-} .
$$

From Lemma 4.2 and as the eigenvalues, for any $X \in \mathfrak{a}_{I}$, of $E_{\lambda}\left(\Gamma_{\mathcal{I}}(X)\right)$ are pure imaginary if $\lambda \in \mathcal{Q}_{\mathcal{I}}^{0}$, one has that:

The map $T \mapsto \tilde{f}_{I}(\exp (T X))$ is an exponential polynomial with unitary characters.

4.4 Lemma. For any $f \in \mathcal{A}_{\text {temp }, N}(Z: \mathcal{I})$,

$$
\left(L_{a_{I}^{-1}} f\right)_{I}^{\sim}\left(a_{Z}\right)=a_{I}^{\rho_{Q}} \tilde{f}_{I}\left(a_{I} a_{Z}\right), \quad a_{I} \in A_{I}, a_{Z} \in A_{Z}
$$

Proof. Using (3.20), one sees that, for any $a_{Z} \in A_{Z}, a_{I} \in A_{I}$,

$$
<\Phi_{L_{a_{I}^{-1}} f}\left(a_{Z}\right), v>=a_{Z}^{-\rho_{Q}}\left(L_{v^{I}} L_{a_{I}^{-1}} f\right)\left(a_{Z}\right) .
$$


But, as $v^{I} \in Z\left(\mathfrak{l}_{I}\right), L_{a_{I}^{-1}}$ commutes with $L_{v^{I}}$. Hence:

$$
\begin{aligned}
<\Phi_{L_{a_{I}^{-1}} f}\left(a_{Z}\right), v> & =a_{Z}^{-\rho_{Q}}\left(L_{a_{I}^{-1}} L_{v^{I}} f\right)\left(a_{Z}\right) \\
& =a_{I}^{\rho_{Q}} a_{I}^{-\rho_{Q}} a_{Z}^{-\rho_{Q}}\left(L_{v^{I}} f\right)\left(a_{I} a_{Z}\right) \\
& =a_{I}^{\rho_{Q}}<\Phi_{f}\left(a_{I} a_{Z}\right), v> \\
& =a_{I}^{\rho_{Q}}<\left(L_{a_{I}^{-1}} \Phi_{f}\right)\left(a_{Z}\right), v>
\end{aligned}
$$

Hence $\Phi_{L_{a_{I}^{-1}} f}=a_{I}^{\rho_{Q}} L_{a_{I}^{-1}} \Phi_{f}$. Going to the defintition to $\Phi_{f, \lambda, \infty}$ (cf. (3.27) and (3.30)) and of $\tilde{f}_{I}$ (cf. (4.3)), one gets the equality of the Lemma.

Let $\mathcal{C}$ be as in Lemma 4.1. According to this Lemma and the fact that $\Phi_{f, \lambda, \infty}=0$ for $\lambda \in \mathcal{Q}_{\mathcal{I}}^{+} \cup \mathcal{Q}_{\mathcal{I}}^{-}($cf. $(3.30))$ and $\mathcal{C}$ is a compact subset of $\mathfrak{a}_{I}^{--}$, one has:

$$
\begin{array}{r}
\left\|\Phi_{f}\left(a_{Z} \exp (T X)\right)-\Phi_{f, \infty}\left(a_{Z} \exp (T X)\right)\right\| \leqslant c e^{-\varepsilon T} q(f)\left(1+\left\|\log a_{Z}\right\|\right)^{N}, \\
T \geqslant 0, a_{Z} \in A_{Z}^{-}, X \in \mathcal{C},
\end{array}
$$

where $c$ is the cardinal of $\mathcal{Q}_{\mathcal{I}}$. By the property (3.20) of $\Phi_{f}$ applied with $v=1$, one sees that:

$$
<\Phi_{f}\left(a_{z} \exp (T X)\right), 1>=a_{Z}^{-\rho_{Q}} e^{-T \rho_{Q}(X)} f\left(a_{Z} \exp (T X)\right) .
$$

Using the equation above and the definition (4.3) of $\tilde{f}_{I}$, one deduces from (4.5) the following Lemma.

4.5 Lemma. Let $\mathcal{C}$ be as in Lemma 4.1. There exist $c>0, \varepsilon>0$ and a continuous semi-norm $q$ on $\mathcal{A}_{\text {temp }, N}(Z)$ such that, for $f \in \mathcal{A}_{\text {temp }, N}(Z: \mathcal{I}), X \in \mathcal{C}, a_{Z} \in A_{Z}^{-}$and $T \geqslant 0$,

$$
\left|\left(a_{Z} \exp (T X)\right)^{-\rho_{Q}} f\left(a_{Z} \exp (T X)\right)-\tilde{f}_{I}\left(a_{Z} \exp (T X)\right)\right| \leqslant c e^{-\varepsilon T} q(f)\left(1+\left\|\log a_{Z}\right\|\right)^{N} .
$$

Let us show that, for any $X \in \mathfrak{a}_{I}^{--}$,

$$
\lim _{T \rightarrow \infty}\left(\left(a_{Z_{I}} \exp (T X)\right)^{-\rho_{Q}} f\left(a_{Z_{I}} \exp (T X)\right)-\tilde{f}_{I}\left(a_{Z_{I}} \exp (T X)\right)\right)=0, \quad a_{Z_{I}} \in A_{Z_{I}}^{-} .
$$

If $a_{Z_{I}} \in A_{Z}^{-}$, it follows from Lemma 4.5. If $a_{Z_{I}} \in A_{Z_{I}}^{-}$, one writes $a_{Z_{I}} \exp (T X)=$ $a_{Z_{I}} \exp \left(T_{0} X\right) \exp \left(\left(T-T_{0}\right) X\right)$, where $T_{0}>0$ is such that $a_{Z_{I}} \exp \left(T_{0} X\right) \in A_{Z}^{-}$. Then one uses Lemma 4.5 and obtains (4.6).

\subsection{Definition of the constant term of elements of $\mathcal{A}_{\text {temp }}(Z: \mathcal{I})$}

Let us first start by the following general remark:

If an exponential polynomial function of one variable, $P(t)$, with unitary characters, satisfies:

$$
\lim _{t \rightarrow+\infty} P(t)=0
$$

then $P \equiv 0$. 
We define some linear forms $\eta$ and $\eta_{I}$ on $\mathcal{A}_{\text {temp }}(Z: \mathcal{I})$ by:

$$
\begin{aligned}
<\eta, f> & =f\left(z_{0}\right) \\
<\eta_{I}, f> & =\tilde{f}_{I}\left(z_{0, I}\right), \quad f \in \mathcal{A}_{\text {temp }}(Z: \mathcal{I})
\end{aligned}
$$

Let us remark that $\eta$ is a continuous linear form on $\mathcal{A}_{\text {temp,N }}(Z: \mathcal{I})$.

4.6 Lemma. With $f$ as above, one has:

$$
m_{\eta_{I}, f}(a)=a^{\rho_{Q}} \tilde{f}_{I}(a), \quad a \in A_{I} .
$$

Proof. This follows from the definition of $\eta_{I}$ and Lemma 4.4 for $a_{I}=a$ and $a_{Z}=1$.

4.7 Lemma. The linear form $\eta_{I}$ is the unique linear form on $\mathcal{A}_{\text {temp }, N}(Z: \mathcal{I})$ such that:

(i) $\lim _{T \rightarrow \infty}(\exp (T X))^{-\rho_{Q}}\left(m_{\eta, f}(\exp (T X))-m_{\eta_{I}, f}(\exp (T X))\right)=0, \quad f \in \mathcal{A}_{\text {temp }, N}(Z$ : $\mathcal{I}), X \in \mathfrak{a}_{I}^{--}$.

(ii) For any $X \in \mathfrak{a}_{I}, T \mapsto(\exp (T X))^{-\rho_{Q}} m_{\eta_{I}, f}(\exp (T X))$ is an exponential polynomial with unitary characters.

Moreover $\eta_{I}$ is continuous and $H_{I}$-invariant.

Proof. The assertion (i) follows from Lemma 4.6 and (4.6). From Lemma 4.6 and (4.4), one gets (ii).

To prove the unicity of such an $\eta_{I}$ satisfying (i) and (ii), we use (4.7). If $\eta_{I}^{\prime}$ is another linear form satisfying (i) and (ii), then, for any $f \in \mathcal{A}_{\text {temp }, N}(Z: \mathcal{I})$,

$$
m_{\eta_{I}, f}(\exp (T X))-m_{\eta_{I}^{\prime}, f}(\exp (T X))=0, \quad X \in \mathfrak{a}_{I}^{--}, T \in \mathbb{R} .
$$

This equality applied to $T=0$ implies that $\eta_{I}=\eta_{I}^{\prime}$.

Let us show the continuity of $\eta_{I}$. By taking $T=0$ and $a_{Z}=1$ in the inequality of Lemma 4.5, one gets:

$$
\left|f\left(z_{0}\right)-\tilde{f}_{I}\left(z_{0, I}\right)\right| \leqslant C q(f) \text {, i.e. }\left|<\eta, f>-<\eta_{I}, f>\right| \leqslant C q(f) .
$$

Moreover $\eta$ is a continuous map on $\mathcal{A}_{\text {temp }, N}(Z: \mathcal{I})$. This implies that $\eta_{I}$ is continuous on $\mathcal{A}_{\text {temp }, N}(Z: \mathcal{I})$.

It remains to get that $\eta_{I}$ is $H_{I^{-}}$-invariant. From (4.6), for any $X \in \mathfrak{a}_{I}^{--}$,

$$
\lim _{T \rightarrow \infty}\left((\exp (T X))^{-\rho_{Q}} f(\exp (T X))-\tilde{f}_{I}(\exp (T X))\right)=0 .
$$

One applies this to $L_{Y} f, Y \in \mathfrak{h}_{I}$, and gets:

$$
\lim _{T \rightarrow \infty}\left(\exp (T X)^{-\rho_{Q}} L_{Y} f(\exp (T X))-\left(L_{Y} f\right)_{I}^{\sim}(\exp (T X))\right)=0 .
$$


On the other hand, from Lemma 3.1, one has:

$$
\lim _{T \rightarrow \infty} \exp (T X)^{-\rho_{Q}} L_{Y} f(\exp (T X))=0
$$

Hence, one gets from (4.8) and (4.9) that:

$$
\lim _{T \rightarrow \infty}\left(L_{Y} f\right)_{I}^{\sim}(\exp (T X))=0 .
$$

But $T \mapsto\left(L_{Y} f\right)_{I}^{\sim}(\exp (T X))$ is an exponential polynomial with unitary characters (cf. (4.4)). Hence, from (4.7), it is identically equal to 0 . This means that:

$$
\eta_{I}\left(L_{Y} f\right)=0
$$

Then $\eta_{I}$ is continuous and $\mathfrak{h}_{I}$-invariant, and hence $H_{I^{-}}$-invariant.

For $f \in \mathcal{A}_{\text {temp,N }}(Z: \mathcal{I})$, let $f_{I}$ be the function on $Z_{I}$ defined by:

$$
f_{I}\left(g \cdot z_{0, I}\right)=m_{\eta_{I}, f}(g), \quad g \in G .
$$

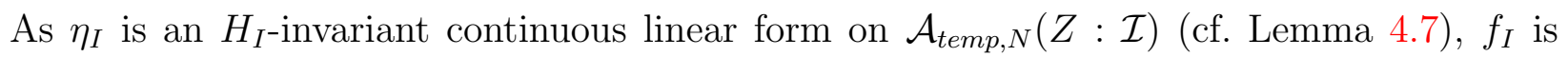
well-defined. Moreover,

$$
\left(L_{g} f\right)_{I}=L_{g} f_{I}, \quad g \in G \text {. }
$$

4.8 Proposition. Let $f \in \mathcal{A}_{\text {temp }, N}(Z: \mathcal{I})$. One has that $f_{I}$ is the unique $C^{\infty}$ function on $Z_{I}$ such that, for all $g \in G$ :

(i) For $X \in \mathfrak{a}_{I}^{--}, \lim _{T \rightarrow \infty}(\exp (T X))^{-\rho_{Q}}\left(f(g \exp (T X))-f_{I}(g \exp (T X))\right)=0$,

(ii) For $X \in \mathfrak{a}_{I}, T \mapsto(\exp (T X))^{-\rho_{Q}} f_{I}(g \exp (T X))$ is an exponential polynomial with unitary characters.

Proof. The Proposition follows immediately from Lemma 4.7 applied to $L_{g^{-1}} f,(4.11)$ and the definition (4.10) of $f_{I}$. Unicity follows from (4.7).

4.9 Lemma. For any $f$ as above:

$$
f_{I}\left(a_{Z_{I}}\right)=a_{Z_{I}}^{\rho_{Q}} \tilde{f}_{I}\left(a_{Z_{I}}\right), \quad a_{Z_{I}} \in A_{Z_{I}}^{-}
$$

Proof. This follows from Proposition 4.8 and (4.6).

4.10 Lemma. Let $p$ be as in Lemma 4.3. For any $a_{Z_{I}} \in A_{Z_{I}}^{-}$and $f \in \mathcal{A}_{\text {temp,N }}(Z: \mathcal{I})$,

$$
\left|f_{I}\left(a_{Z_{I}}\right)\right| \leqslant a_{Z_{I}}^{\rho_{Q_{I}}} p(f)\left(1+\left\|\log a_{Z_{I}}\right\|\right)^{N+\operatorname{dim} W_{\mathcal{I}}} .
$$


Proof. The Lemma follows from Lemma 4.3, (4.3) and (4.12).

Let $w_{I} \in \mathcal{W}_{I}$ (the set analogous to $\mathcal{W}$ for $Z_{I}$ ). Let $w$ be the element of $\mathcal{W}$ associated to $w_{I}$ by $[11$, Lemma 3.10].

Set $H_{I, w_{I}}=w_{I} H_{I} w_{I}^{-1}$ and $H_{w}=w H w^{-1}$. Consider the real spherical spaces $Z_{w}=G / H_{w}$ and $Z_{I, w_{I}}=G / H_{I, w_{I}}$, and put $z_{0}^{w}=H_{w} \in Z_{w}$ and $z_{0, I}^{w_{I}}=H_{I, w_{I}} \in Z_{I, w_{I}}=G / H_{I, w_{I}}$. Then (cf. [11, Corollary 3.7]) $Q$ is $Z_{w}$-adapted to $P$ and $\mathfrak{a}_{Z}^{-}$is the compression cone for $Z_{w}$.

For $f \in C^{\infty}(Z)$, let us define $f^{w}$ by:

$$
f^{w}\left(g \cdot z_{0}^{w}\right)=f\left(g w \cdot z_{0}\right), \quad g \in G .
$$

In the same way, one defines $\phi^{w_{I}}$ for $\phi \in C^{\infty}\left(Z_{I}\right)$. Then $f^{w} \in C^{\infty}\left(Z_{w}\right)$ and $\phi^{w_{I}} \in C^{\infty}\left(Z_{I, w_{I}}\right)$.

Let $I \subset S$. Let us choose $X_{I} \in \mathfrak{a}_{I}^{--}$, i.e. $X_{I} \in \mathfrak{a}_{I}$ and $\alpha\left(X_{I}\right)<0$ for all $\alpha \in S \backslash I$. For $s \in \mathbb{R}$, let

$$
a_{s}:=\exp \left(s X_{I}\right) .
$$

Let $w_{I} \in \mathcal{W}_{I}$. From Lemma 1.1 applied to the real spherical space $Z_{I}$, one has:

$$
w_{I}=\tilde{t}_{I} h_{I}, \quad \text { for some } \tilde{t}_{I} \in \exp \left(i \tilde{\mathfrak{a}}_{Z}\right) \text { and } h_{I} \in H_{I, \mathbb{C}, 0},
$$

One has PwH open (cf. (1.5)) and there exists $s_{0}>0$ with

$$
P w_{I} a_{s} H=P w H, \quad s \geqslant s_{0} .
$$

One has (cf. Lemma 1.1):

$$
w=\tilde{t} h \quad \text { for some } \tilde{t} \in \exp \left(i \tilde{\mathfrak{a}}_{Z}\right) \text { and } h \in H_{\mathbb{C}, 0} .
$$

For any $s \geqslant s_{0}$, let $u_{s} \in U, b_{s} \in A_{Z}, m_{s} \in M$ and $h_{s} \in H$ be given by loc.cit. Lemma 3.10. In particular:

$$
\begin{aligned}
w_{I} \tilde{a}_{s} & =u_{s} m_{s} \tilde{b}_{s} w h_{s}, \quad s \geqslant s_{0}, \\
\lim _{s \rightarrow+\infty}\left(a_{s} b_{s}^{-1}\right) & =1, \\
\lim _{s \rightarrow+\infty} u_{s} & =1, \\
\lim _{s \rightarrow+\infty} m_{s} & =m_{w_{I}}, \text { for some } m_{w_{I}} \in M .
\end{aligned}
$$

Let us notice that (4.16) is valid without assuming $Z$ wave-front or unimodular.

Let us remark that:

$$
\text { If } w_{I}=1 \text {, one can take } w=1 \text { and then one has } m_{w_{I}}=1 \text {. }
$$

The proof of the following Proposition will be postponed to the next Section.

4.11 Proposition. Let $w_{I} \in \mathcal{W}_{I}, w \in \mathcal{W}$ be as above and $f \in \mathcal{A}_{\text {temp }, N}(Z: \mathcal{I})$. Then $f^{w} \in \mathcal{A}_{\text {temp }, N}\left(Z_{w}: \mathcal{I}\right)$ and

$$
\left(L_{m_{w_{I}}} f_{I}\right)^{w_{I}}\left(a_{Z}\right)=\left(f^{w}\right)_{I}\left(a_{Z}\right), \quad a_{Z} \in A_{Z} .
$$


Here $f^{w} \in \mathcal{A}_{\text {temp }}\left(Z_{w}: \mathcal{I}\right),\left(f^{w}\right)_{I} \in C^{\infty}\left(Z_{w, I}\right), f_{I} \in C^{\infty}\left(Z_{I}\right),\left(L_{m_{w_{I}}} f_{I}\right)^{w_{I}} \in C^{\infty}\left(Z_{I, w_{I}}\right)$, and, from [11, Proposition 3.2(5) and Corollary 3.7], one has:

$$
\begin{aligned}
& A_{Z_{w, I}}=A_{Z_{w}}=A_{Z}, \\
& A_{Z_{I, w_{I}}}=A_{Z_{I}}=A_{Z} .
\end{aligned}
$$

Hence both sides of the equality are well-defined on $A_{Z}$.

Before stating the next Theorem, we recall, from Proposition 4.8, that, for $f \in \mathcal{A}_{\text {temp }}(Z)$, $f_{I}$ is the unique $C^{\infty}$ function on $Z_{I}$ such that, for all $X \in \mathfrak{a}_{I}^{--}$and $g \in G$,

$$
\lim _{T \rightarrow \infty}(\exp (T X))^{-\rho_{Q}}\left(f(g \exp (T X))-f_{I}(g \exp (T X))\right)=0
$$

and

$$
\begin{aligned}
& T \mapsto(\exp T X)^{-\rho_{Q}} f_{I}(g \exp (T X)) \text { is an exponential polynomial } \\
& \text { with unitary characters. }
\end{aligned}
$$

We see that, using Lemma 4.2 for $\lambda \in \mathcal{Q}_{\mathcal{I}}^{0}$, one can replace (4.19) by the stronger condition:

$$
X \mapsto(\exp X)^{-\rho_{Q}} f_{I}(g \exp X) \text { is an exponential polynomial on } \mathfrak{a}_{I}
$$

Let $f \in \mathcal{A}_{\text {temp }}(Z)$. Then $f \in \mathcal{A}_{\text {temp }, N}(Z: \mathcal{I})$ for some $N$ as above and some finite codimensional ideal $\mathcal{I}$ in $Z(\mathfrak{g})$. Hence we can define $f_{I}$ as above.

4.12 Proposition. With $f \in \mathcal{A}_{\text {temp }}(Z)$ as above, one has that $f_{I}$ does not depend on $N$ and $\mathcal{I}$.

Proof. This follows from the characterization of $f_{I}$ above (see (4.18) and (4.19)).

From this Proposition, we can define a linear form, still denoted $\eta_{I}$, on $\mathcal{A}_{\text {temp }}(Z)$, by $f \mapsto f_{I}\left(z_{0, I}\right)$.

\subsection{Theorem.}

(i) With $N_{\mathcal{I}}=\operatorname{dim} W_{\mathcal{I}}$ as in Lemma 4.10, for all $N \in \mathbb{N}$, the map $f \mapsto f_{I}$ is a continuous linear map from $\mathcal{A}_{\text {temp }, N}(Z: \mathcal{I})$ to $\mathcal{A}_{\text {temp }, N+N_{\mathcal{I}}}\left(Z_{I}: \mathcal{I}\right)$.

(ii) Let $N \in \mathbb{N}, \mathcal{C}$ be a compact subset of $\mathfrak{a}_{I}^{--}$and $\Omega^{\prime}$ be a compact subset of $G$. Let $w_{I} \in \mathcal{W}_{I}$ and $\left(w, m_{w_{I}}\right) \in \mathcal{W} \times M$ be as above. Then there exist $\varepsilon>0$ and a continuous semi-norm $p$ on $C_{\text {temp }, N}^{\infty}(Z)$ such that, for all $f \in \mathcal{A}_{\text {temp }, N}(Z: \mathcal{I})$,

$$
\begin{aligned}
& \left|\left(a_{Z} \exp (T X)\right)^{-\rho_{Q}}\left(f\left(\omega^{\prime} a_{Z} \exp (T X) w \cdot z_{0}\right)-f_{I}\left(\omega^{\prime} m_{w_{I}}^{-1} a_{Z} \exp (T X) w_{I} \cdot z_{0, I}\right)\right)\right| \\
& \leqslant e^{-\varepsilon T} p(f)\left(1+\left\|\log a_{Z}\right\|\right)^{N}, \quad a_{Z} \in A_{Z}^{-}, X \in \mathcal{C}, \omega^{\prime} \in \Omega^{\prime}, T \geqslant 0 .
\end{aligned}
$$


Proof. In view of (2.6), to get (i), it is enough to prove that, for any $w_{I} \in \mathcal{W}_{I}$ and any compact subset $\Omega$ of $G$, there exists a continuous semi-norm $p$ on $\mathcal{A}_{\text {temp }, N}(Z: \mathcal{I})$ such that:

$$
\sup _{\omega \in \Omega, a \in A_{Z_{I}^{-}}^{-}}\left|a^{-\rho_{Q}}(1+\log \|a\|)^{-\left(N+N_{I}\right)} f_{I}\left(\omega a w_{I}\right)\right| \leqslant p(f), \quad f \in \mathcal{A}_{t e m p, N}(Z: \mathcal{I}) .
$$

Using (2.7), one is reduced to prove this for $\Omega$ reduced to 1 . For $w_{I}=1$, one can take $w=1$ (cf. (4.17)) and our claim follows from Lemma 4.10. For general $w_{I}$, one uses Proposition 4.11 to get $\left(L_{m_{w_{I}}} f_{I}\right)\left(a_{Z_{I}} w_{I}\right)=\left(f^{w}\right)_{I}\left(a_{Z_{I}}\right)$ and the above inequality for $H^{w}$. This shows (i).

One reduces easily to prove (ii) for $\Omega=\{1\}$, by using (2.7). Then, using Proposition 4.11, one is reduced to prove (ii) with $\Omega=\{1\}$ and $w_{I}=w=m_{w_{I}}=1$ by changing $H$ into $H_{w}$. In that case, (ii) follows from Lemma 4.1.

\subsection{Constant term of tempered $H$-fixed linear forms}

Let $I$ be a subset of $S$.

4.14 Proposition. Let $\left(\pi, V^{\infty}\right)$ be a Harish-Chandra $G$-representation. If $\xi$ is a Z-tempered continuous linear form on $V$, then there exists a unique $Z_{I}$-tempered continuous linear form $\xi_{I}$ on $V^{\infty}$ such that:

(i) $\lim _{T \rightarrow \infty}(\exp (T X))^{-\rho_{Q}}\left(m_{\xi, v}(\exp (T X))-m_{\xi_{I}, v}(\exp (T X))\right)=0, v \in V^{\infty}, X \in \mathfrak{a}_{I}^{--}$.

(ii) For any $v \in V^{\infty}$ and $X \in \mathfrak{a}_{I}, T \mapsto(\exp (T X))^{-\rho_{Q}} m_{\xi_{I}, v}(\exp (T X))$ is an exponential polynomial with unitary characters.

Proof. Let

$$
<\xi_{I}, v>:=\left(m_{\xi, v}\right)_{I}\left(z_{0, I}\right), \quad v \in V^{\infty}
$$

Then

$$
\begin{aligned}
m_{\xi_{I}, v}(g) & =<\xi_{I}, \pi\left(g^{-1}\right) v> \\
& =\left(m_{\xi, \pi\left(g^{-1}\right) v}\right)_{I}\left(z_{0, I}\right) \\
& =\left(L_{g^{-1}} m_{\xi, v}\right)_{I}\left(z_{0, I}\right) .
\end{aligned}
$$

As $f \mapsto f_{I}$ is a $G$-morphism (cf. Theorem 4.13), one then obtains that:

$$
m_{\xi_{I}, v}\left(g \cdot z_{0, I}\right)=\left(m_{\xi, v}\right)_{I}\left(g \cdot z_{0, I}\right) .
$$

From the properties of $\left(m_{\xi, v}\right)_{I}$, one sees that (ii) is satisfied. Furthermore, from Theorem 4.13, one sees that $\left(m_{\xi, v}\right)_{I} \in \mathcal{A}_{\text {temp }, N}\left(Z_{I}\right)$ for some integer $N$. Hence $\xi_{I}$ satisfies the required properties. Unicity is clear using (4.7). 


\subsection{Application to the relative discrete series for $Z$}

As $Z$ is wave-front and $\rho_{Q} \in \mathfrak{a}_{Z}$, one has $\rho_{Q \mid \mathfrak{a}_{Z}^{-}} \leqslant 0$. Hence $\rho_{Q \mid \mathfrak{a}_{Z, E}}=0$.

Let $\chi$ be a unitary character of $A_{Z, E}$. We recall that, if $a \in A_{Z, E}$ and $w \in \mathcal{W}, \tilde{a} w H=w a$ (cf. [11, Lemma 3.5]). As $A_{Z, E}$ normalizes $H$, there is a right action $(a, z) \mapsto z \cdot a$ of $A_{Z, E}$ on $Z$. Let $C^{\infty}(Z, \chi)$ be the space of $C^{\infty}$ functions on $Z$ such that:

$$
f(z \cdot a)=\chi(a) f(z), \quad a \in A_{Z, E}, z \in Z .
$$

If $f \in C^{\infty}(Z, \chi), u \in U(\mathfrak{g})$ and $N \in \mathbb{N}$, let

$$
r_{N, u}(f)=\sup _{\omega \in \Omega, a \in A_{\bar{Z}}^{-} / A_{Z, E}, w \in \mathcal{W}}\left|a^{-\rho_{Q}}(1+\|\log a\|)^{N}\left(L_{u} f\right)(\omega a w)\right|,
$$

and we define:

$$
\mathcal{C}(Z, \chi)=\left\{f \in C^{\infty}(Z, \chi): r_{N, u}(f)<\infty, N \in \mathbb{N}, u \in U(\mathfrak{g})\right\} .
$$

Let us recall that $\widehat{H}=H A_{Z, E}$ and $\widehat{Z}=G / \widehat{H}$. If $\chi$ is a character of $A_{Z, E}$, we extend it trivially to $H$ on a character of $\hat{H}$ still denoted $\chi$. Let us define $L^{2}(\widehat{Z} ; \chi)$ as in [11, Section 8.1], by replacing $\chi$ by $\chi^{-1}$.

4.15 Theorem. Let $\left(\pi, V^{\infty}\right)$ be a Harish-Chandra $G$-representation and $\eta$ be a Z-tempered continuous linear form on $V^{\infty}$ which transforms under a character $\chi$ of $A_{Z, E}$. Then the following assertions are equivalent:

(i) For all $v \in\left(V^{\infty}\right)_{(K)}, m_{\eta, v} \in L^{2}(\hat{Z} ; \chi)$.

(ii) For all proper subset I of $S, \eta_{I}=0$.

(iii) For all $v \in V^{\infty}, m_{\eta, v} \in \mathcal{C}(Z, \chi)$.

4.16 Remark. Note that we use $\chi^{-1}$ instead of $\chi$ in [11] as we use that the linear form $\eta$ transforms by $\chi$ under the natural action of $A_{Z, E}$ on the dual of $V^{\infty}$.

Proof. Let us assume (i). Let $S=\left\{\sigma_{1}, \ldots, \sigma_{s}\right\}$ and $\omega_{1}, \ldots, \omega_{s} \in \mathfrak{a}_{Z}$ be such that:

$$
\begin{array}{ll}
\sigma_{i}\left(\omega_{j}\right)=\delta_{i, j}, & i, j=1, \ldots, s \\
\omega_{i} \perp \mathfrak{a}_{Z, E}, & i=1, \ldots, s .
\end{array}
$$

Here we use the scalar product on $\mathfrak{a}_{Z}$ defined before (1.4). From [11, Theorem 8.5], the linear form $\Lambda_{V, \eta}$ on $\mathfrak{a}_{Z}$, defined in loc.cit. (6.10), satisfies

$$
\left(\Lambda_{V, \eta}-\rho_{Q}\right)\left(\omega_{j}\right)>0, \quad j=1, \ldots, s .
$$

Then it follows from loc.cit. Theorem 7.6 used for a fixed $X \in \mathfrak{a}_{Z}^{-}$of norm $1, \Omega=\{\exp (-X)\}$, $w=1$ and $t=1$, that there exists a $d \in \mathbb{N}$ and a continuous semi-norm $p$ on $V^{\infty}$ such that:

$$
\left|m_{\eta, v}(a)\right| \leqslant p(v) a^{\Lambda_{V, \eta}}(1+\|\log a\|)^{d}, \quad a \in A_{Z}^{-}, v \in V,
$$


where $V=\left(V^{\infty}\right)_{(K)}$. Let $I$ be a proper subset of $S$ and $X_{I}=-\sum_{i, \omega_{i} \in S \backslash I} \omega_{i} \in \mathfrak{a}_{I}^{--}$. From (4.20), one deduces that one can choose $\beta>0$ such that:

$$
\left(\Lambda_{V, \eta}-\rho_{Q}\right)\left(X_{I}\right)<-\beta
$$

Hence, one deduces from (4.21) that, for each $v \in V$,

$$
\left|m_{\eta, v}\left(\exp \left(t X_{I}\right)\right)\right| \leqslant p(v) e^{t \rho_{Q}\left(X_{I}\right)}\left(1+t\left\|X_{I}\right\|\right)^{d} e^{-t \beta}, \quad t \geqslant 0 .
$$

As $\beta>0$, this implies that:

$$
\lim _{t \rightarrow+\infty}\left(\exp \left(t X_{I}\right)\right)^{-\rho_{Q}} m_{\eta, v}\left(\exp \left(t X_{I}\right)\right)=0 .
$$

From the definition of the constant term $\eta_{I}$ of $\eta$ (cf. Proposition 4.14) and from (4.7), one deduces $\eta_{I}(v)=0$ for any $v \in V$. As $\eta_{I}$ is continuous on $V^{\infty}$ and $V$ is dense in $V^{\infty}$, one concludes $\eta_{I}=0$. This achieves to prove that (i) implies (ii).

Let us assume that (ii) holds. Let $\mathcal{I}$ be an ideal of $Z(\mathfrak{g})$ which annihilates $V$ or $V^{\infty}$. It is of finite codimension. Let us assume that, for all $v \in V^{\infty}, m_{\eta, v} \in \mathcal{A}_{t e m p, N}(Z: \mathcal{I})$. Then one can apply Theorem 4.13. Let $v \in V^{\infty}$ and set $f=m_{\eta, v}$. Let $I \mp S$. Let $\mathcal{C}$ be a compact subset of $\mathfrak{a}_{I}^{--}, \Omega_{1}$ be a compact subset of $G$ and $u \in U(\mathfrak{g})$. Hence there exists a continuous semi-norm $p$ on $\mathcal{A}_{t e m p, N}(Z), \varepsilon>0$ such that:

$$
\begin{aligned}
& \left|\left(a_{Z} \exp (T X)\right)^{-\rho_{Q}}\left(L_{u} f\right)\left(\omega a_{Z} \exp (T X) w \cdot z_{0}\right)\right| \\
& \leqslant e^{-\varepsilon T} p(f)\left(1+\left\|\log a_{Z}\right\|\right)^{N}, \quad a_{Z} \in A_{Z}^{-} / A_{Z, E}, X \in \mathcal{C}, \omega \in \Omega_{1}, w \in \mathcal{W}, T \geqslant 0 .
\end{aligned}
$$

From this, we will deduce that $f \in \mathcal{C}(Z, \chi)$. Let $S_{1}$ be the unit sphere on $\mathfrak{a}_{Z} / \mathfrak{a}_{Z, E}$ and let $X_{0} \in S_{1} \cap \mathfrak{a}_{Z}^{-} / \mathfrak{a}_{Z, E}$. Let $\Omega_{0}$ be an open neighborhood of $X_{0}$ in $S_{1} \cap \mathfrak{a}_{Z}^{-} / \mathfrak{a}_{Z, E}$ such that, for all $X \in \Omega_{0}, \alpha(X) \leqslant \alpha\left(X_{0}\right) / 2, \alpha \in S$. Let $I$ be the set of $\alpha \in S$ such that $\alpha\left(X_{0}\right)=0$. One has $I \neq S$. Then one has $X_{0} \in \mathfrak{a}_{I}^{--}$. Let $Y \in \Omega_{0}$ and $t \geqslant 0$. Then $t\left(Y-X_{0} / 2\right) \in \mathfrak{a}_{Z}^{-}$and $\exp (t Y)=\exp t\left(Y-X_{0} / 2\right) \exp \left(t X_{0} / 2\right)$. Using (4.22) for $X_{0} / 2$ instead of $X$, $\exp t\left(Y-X_{0} / 2\right)$ instead of $a_{Z}$ and $T=t$, one gets:

$$
\begin{aligned}
& \left|(\exp (t Y))^{-\rho_{Q}}\left(L_{u} f\right)\left(\omega \exp (t Y) w \cdot z_{0}\right)\right| \\
& \leqslant e^{-\varepsilon t} p(f)\left(1+t\left\|Y-X_{0} / 2\right\|\right)^{N}, \quad Y \in \Omega_{0}, \omega \in \Omega_{1}, w \in \mathcal{W}, t \geqslant 0 .
\end{aligned}
$$

One deduces easily from this that:

$$
\sup _{\omega \in \Omega_{1}, w \in \mathcal{W}, a \in \exp \left(\mathbb{R}^{+} \Omega_{0}\right)} a^{-\rho_{Q}}(1+\|\log a\|)^{N}\left|\left(L_{u} f\right)\left(\omega a w \cdot z_{0}\right)\right|<+\infty .
$$

Using a finite covering of the compact set $S_{1} \cap \mathfrak{a}_{Z}^{-} / \mathfrak{a}_{Z, E}$, one deduces from this that $f \in \mathcal{C}(Z, \chi)$. This achieves to prove that (ii) implies (iii).

To prove that (iii) implies (i), one proceeds as in the proof that (ii) implies (i) in [11, Theorem 8.5]. 


\section{$5 \quad$ Proof of Proposition 4.11}

We refer to Section B for the definition and properties of rapid convergence. The main goal of this Section is to prove:

5.1 Proposition. Here we only assume $Z$ real spherical (not necessarily wave-front or unimodular). The families $\left(a_{s} b_{s}^{-1}\right)$ and $\left(u_{s}\right)$ converge rapidly to 1 and one can choose the family $\left(m_{s}\right)$ such that $\left(m_{s}\right)$ converges rapidly to $m_{w_{I}}$.

Let $w_{I} \in \mathcal{W}_{I}$ and $w \in \mathcal{W}$ corresponding to $w_{I}$ as in [11, Lemma 3.10]. In particular, there exists $s_{0}>0$ with $P w_{I} a_{s} H=P w H, s \geqslant s_{0}$. Then one introduces $u_{s} \in U, b_{s} \in A_{Z}, m_{s} \in M$ and $h_{s} \in H$ as in loc.cit. Lemma 3.10 (cf. (4.16)).

\subsection{Reduction of the proof of Proposition 5.1 to the case where $Z$ is quasi-affine}

First we will reduce the proof to the case where $Z$ is quasi-affine.

Let $\underline{H}$ be the connected algebraic group defined over $\mathbb{R}$ with Lie algebra $\mathfrak{h}$. Let us recall that $Z=G / H$ is quasi-affine if $\underline{Z}=\underline{G} / \underline{H}$ is quasi-affine (this is equivalent to suppose that there is an embedding of $\underline{G} / \underline{H}$ in an affine space $V$ defined over $\mathbb{R}$ ).

Hence let us assume that the Proposition has been proved when $Z$ is quasi-affine. We want to prove it for a general $Z$.

Given a real spherical space $G / H$, we want to associate a quasi-affine real spherical space $Z^{\prime}=G^{\prime} / H^{\prime}$.

From [3, Theorem 11.2], there exists a rational representation of $\underline{G},(\pi, \underline{V})$, which is immersive, defined over $\mathbb{R}$ and such that there is a line $\ell=\mathbb{C} v$, defined over $\mathbb{R}$, such that:

$$
\begin{aligned}
\underline{H}(\mathbb{C}) & =\{x \in \underline{G}(\mathbb{C}): \pi(x) \ell \subset \ell\} \\
\underline{\mathfrak{h}}_{\mathbb{C}} & =\left\{X \in \mathfrak{g}_{\mathbb{C}}: \pi(X) \ell \subset \ell\right\} .
\end{aligned}
$$

We denote by $\psi^{-1}$ the algebraic character of $\underline{H}$ defined over $\mathbb{R}$ by which $\underline{H}(\mathbb{C})$ acts on $v$. Now we let, for $\mathrm{F}=\mathbb{R}$ or $\mathbb{C}$,

$$
\begin{aligned}
\underline{G}^{\prime}(\mathrm{F}) & =\underline{G}(\mathrm{~F}) \times F^{\times} \\
\underline{H}^{\prime} & (\mathrm{F})=\left\{(h, \psi(h)): h \in \underline{H}^{\prime}(\mathrm{F})\right\} .
\end{aligned}
$$

Then the map

$$
(g, z) \underline{H}^{\prime}(\mathbb{C}) \mapsto z \pi(g) v
$$

is an embedding of $\underline{Z^{\prime}}(\mathbb{C}):=\underline{G}^{\prime}(\mathbb{C}) / \underline{H^{\prime}}(\mathbb{C})$ in $\underline{V}$ defined over $\mathbb{R}$. Then, with our convention, $Z^{\prime}=G^{\prime} / H^{\prime}$ is a quasi-affine real spherical space, where $H^{\prime}=\{(h, \psi(h)): h \in H\}$ and $G^{\prime}=G \times \mathbb{R}^{\times}$.

If $P^{\prime}=P \times \mathbb{R}^{\times}$, then it is easily seen that $P^{\prime} H^{\prime}$ is open in $G$. Let us prove the following Lemma.

We thank R. Beuzard-Plessis for his help for the proof of the following Lemma. 


\subsection{Lemma.}

(i) The parabolic $Q^{\prime}:=Q \times \mathbb{R}^{\times}$of $G^{\prime}$ is $Z^{\prime}$-adapted to $P^{\prime}$.

(ii) There is a canonical exact sequence $0 \rightarrow\{0\} \times \mathbb{R} \rightarrow \mathfrak{a}_{Z^{\prime}} \rightarrow \mathfrak{a}_{Z} \rightarrow 0$.

(iii) The set $\mathfrak{a}_{Z^{\prime}}^{-}$is invariant by translation by the image of $\{0\} \times \mathbb{R}$ in $\mathfrak{a}_{Z^{\prime}}$ and projects onto $\mathfrak{a}_{Z}^{-}$.

(iv) If $Z$ is wave-front, the spherical space $Z^{\prime}$ is also wave-front.

(v) The exact sequence of (ii) induces the following exact sequence

$$
0 \rightarrow\{0\} \times \mathbb{R} \rightarrow \mathfrak{a}_{Z^{\prime}, E} \rightarrow \mathfrak{a}_{Z, E} \rightarrow 0
$$

Proof. To get (i), one has to check the conditions (1) to (5) in [10, Theorem 2.3]. Let $L^{\prime}:=L \times \mathbb{R}^{\times}$. First, let us consider the map:

$$
\begin{aligned}
Q^{\prime} \times_{L^{\prime}}\left(L^{\prime} / L^{\prime} \cap H^{\prime}\right) & \rightarrow Z^{\prime} \\
\left(q^{\prime}, l^{\prime} L^{\prime} \cap H^{\prime}\right) & \mapsto q^{\prime} l^{\prime} H^{\prime},
\end{aligned}
$$

and let us show that it is a diffeomorphism. This reduces easily to prove the injectivity which is equivalent to $Q^{\prime} \cap H^{\prime}=L^{\prime} \cap H^{\prime}$. But $(q, s) \in H^{\prime}$ with $q \in Q$ and $s \in \mathbb{R}^{\times}$is equivalent to $q \in Q \cap H$ and $s=\psi(q)$. But then, by the local structure theorem for $Z$, one has $q \in L \cap H$. Hence $(q, s)=(l, \psi(l))$ with $l \in L \cap H$, and hence $(q, s) \in L^{\prime} \cap H^{\prime}$. Hence $Q^{\prime} \cap H^{\prime} \subset L^{\prime} \cap H^{\prime}$ and the reverse inclusion is clear. This proves (1) and (2) of loc.cit. Theorem 2.3.

Let us notice that $L_{n}^{\prime}=L_{n} \times\{1\}$. Indeed, as $L_{n}$ is a product of connected semisimple Lie groups, $\psi_{\mid L_{n}}=1$. Hence, as $L_{n} \subset H, L_{n}^{\prime} \subset H^{\prime}$ which proves condition (3) of loc.cit. Theorem 2.3.

Let us look at $\left(L^{\prime} \cap P^{\prime}\right)\left(L^{\prime} \cap H^{\prime}\right)$. One has $\{1\} \times \mathbb{R}^{\times} \subset L^{\prime} \cap P^{\prime}$. Hence

$$
((L \cap P) \times\{1\})((L \cap H) \times\{1\})\left(\{1\} \times \mathbb{R}^{\times}\right) \subset\left(L^{\prime} \cap P^{\prime}\right)\left(L^{\prime} \cap H^{\prime}\right) .
$$

But, by the local structure theorem for $Z$,

$$
(L \cap P)(L \cap H)=L
$$

Hence, as wanted, we get:

$$
\left(L^{\prime} \cap P^{\prime}\right)\left(L^{\prime} \cap H^{\prime}\right)=L^{\prime}
$$

i.e. condition (4) of loc. cit. Theorem 2.3.

Similarly we get condition (5) of loc.cit. Theorem 2.3, i.e. $Q^{\prime} H^{\prime}=P^{\prime} H^{\prime}$. This finishes to prove (i).

Let us prove (ii). The space $\mathfrak{a}_{Z^{\prime}}$ is the quotient of $\mathfrak{a}_{L^{\prime}}=\mathfrak{a}_{L} \times \mathbb{R}$ by:

$$
\mathfrak{a}_{L^{\prime}} \cap \mathfrak{h}^{\prime}=\left\{(X, \Psi(X)): X \in \mathfrak{a}_{L} \cap \mathfrak{h}\right\}
$$


where $\Psi$ denotes the differential of $\psi$. It is clear that the projection of $\mathfrak{a}_{L^{\prime}}$ on the first factor of $\mathfrak{a}_{L} \times \mathbb{R}$ followed by the projection from $\mathfrak{a}_{L}$ to $\mathfrak{a}_{Z}$ goes through the quotient in a surjective map from $\mathfrak{a}_{Z^{\prime}}$ to $\mathfrak{a}_{Z}$. Its kernel is clearly $\{0\} \times \mathbb{R}$. This proves (ii).

Let us prove (iii). Let $\mathfrak{a}_{Z}^{--}$(resp. $\left.\mathfrak{a}_{Z^{\prime}}^{-}\right)$be the interior of $\mathfrak{a}_{Z}^{-}$(resp. $\left.\mathfrak{a}_{Z^{\prime}}^{-}\right)$. Let $\mathfrak{h}_{\text {lim }}=$ $(\mathfrak{h} \cap \mathfrak{l}) \oplus \mathfrak{u}^{-}$and $\mathfrak{h}_{\text {lim }}^{\prime}=\left(\mathfrak{h}^{\prime} \cap \mathfrak{l}^{\prime}\right) \oplus \mathfrak{u}^{-}$. Then, from [8, Lemma 5.9], one has:

For $X \in \mathfrak{a}_{Z}$ (resp. $\left.X^{\prime} \in \mathfrak{a}_{Z^{\prime}}\right), X \in \mathfrak{a}_{Z}^{--}$(resp. $X^{\prime} \in \mathfrak{a}_{Z^{\prime}}^{-}$) if and only if $\lim _{t \rightarrow+\infty} e^{\operatorname{tad}(X)}(\mathfrak{h})=\mathfrak{h}_{\text {lim }}\left(\right.$ resp. $\left.\lim _{t \rightarrow+\infty} e^{\operatorname{tad}\left(X^{\prime}\right)}\left(\mathfrak{h}^{\prime}\right)=\mathfrak{h}_{\text {lim }}^{\prime}\right)$.

It is clear from (5.1) that $\mathfrak{a}_{Z^{\prime}}^{-}$is invariant by translation by the image of $\{0\} \times \mathbb{R}$ in $\mathfrak{a}_{Z^{\prime}}$.

Let $X^{\prime} \in \mathfrak{a}_{Z^{\prime}}^{--}$and $X$ its projection on $\mathfrak{a}_{Z}$. Let us look at $e^{\operatorname{tad}(X)}(\mathfrak{h})$. It is the projection of $e^{\operatorname{tad}\left(X^{\prime}\right)}\left(\mathfrak{h}^{\prime}\right)$ on the first factor of $\mathfrak{g} \times \mathbb{R}$. Let $\mathcal{O}$ be the open subset of the Grassmanian $\operatorname{Gr}\left(\mathfrak{g}^{\prime}\right)$ of $\mathfrak{g}^{\prime}$, consisting of the subspaces of $\mathfrak{g}^{\prime}$ which do not contain $\{0\} \times \mathbb{R}$. The map from $\mathcal{O}$ to the Grassmanian $\operatorname{Gr}(\mathfrak{g})$ of $\mathfrak{g}$ which associates to $W \in \mathcal{O}$ its image by the projection onto $\mathfrak{g}$ is continuous. Then the second condition of (5.1) implies that $X \in \mathfrak{a}_{Z}^{--}$.

Now let $X \in \mathfrak{a}_{Z}^{-}$and $\tilde{X} \in \mathfrak{a}_{L}$ which projects onto $X \in \mathfrak{a}_{Z}$. Let $X^{\prime}$ be the projection of $(\tilde{X}, 0)$ in $\mathfrak{a}_{Z^{\prime}}$. Hence $X^{\prime}$ projects onto $X$.

We study $e^{\operatorname{tad} X}\left(\mathbb{R} H^{\prime}\right)$ for $H^{\prime}$ element of a basis of $\mathfrak{h}^{\prime}$. For this, we take a basis of $\mathfrak{l}^{\prime} \cap \mathfrak{h}^{\prime}$ and elements of the form $H_{\alpha}^{\prime}=\left(X_{-\alpha}+T\left(X_{-\alpha}\right), \Psi\left(H_{\alpha}\right)\right.$ ) (cf. (1.4) for the definition of $T$ ), where $H_{\alpha} \in \mathfrak{h}$ is equal to $X_{-\alpha}+T\left(X_{-\alpha}\right)$ and $X_{-\alpha}$ describes a basis of $\mathfrak{g}^{-\alpha}$. If $H^{\prime}$ is an elements of $\mathfrak{l}^{\prime} \cap \mathfrak{h}^{\prime}, e^{\operatorname{tad} \tilde{X}} \mathbb{R} H^{\prime}=\mathbb{R} H^{\prime} \subset \mathfrak{h}_{\text {lim }}^{\prime}$. If $H^{\prime}=H_{\alpha}^{\prime}$, then

$$
e^{\operatorname{tad} \tilde{X}} H_{\alpha}^{\prime}=\left(e^{-t \alpha(\tilde{X})} X_{-\alpha}+\sum_{\beta \in \Sigma_{\mathfrak{u}} \cup\{0\}} e^{t \beta(\tilde{X})} X_{\alpha, \beta}, \Psi\left(H_{\alpha}\right)\right) .
$$

Multiplying by $e^{t \alpha(\tilde{X})}$, one gets:

$$
e^{t \alpha(\tilde{X})} e^{t \operatorname{ad} \tilde{X}}\left(H_{\alpha}^{\prime}\right)=\left(X_{-\alpha}+\sum_{\beta \in \Sigma_{\mathfrak{u}} \cup\{0\}} e^{t(\alpha+\beta)(\tilde{X})} X_{\alpha, \beta}, e^{t \alpha(\tilde{X})} \Psi\left(H_{\alpha}\right)\right) .
$$

If $X_{\alpha, \beta} \neq 0,(\alpha+\beta)(\tilde{X})=(\alpha+\beta)(X)<0$, as $\alpha+\beta \in \mathcal{M} \subset \mathfrak{a}_{Z}^{*}$ and $X \in \mathfrak{a}_{Z}^{--}$.

If $\alpha(\tilde{X})<0$, then $e^{t \alpha(\tilde{X})} \Psi\left(H_{\alpha}\right) \underset{t \rightarrow+\infty}{\longrightarrow} 0$ and (5.3) imply

$$
\lim _{t \rightarrow+\infty} e^{\operatorname{tad} \tilde{X}} \mathbb{R} H_{\alpha}^{\prime}=\mathbb{R}\left(X_{-\alpha}, 0\right) \subset \mathfrak{h}_{\text {lim }}^{\prime}
$$

Let us assume $\alpha(\tilde{X})>0$. Then, if $X_{\alpha, \beta} \neq 0$, one has $(\alpha+\beta)(\tilde{X})<0$ and $\alpha(\tilde{X})>0$. Hence $\beta(\tilde{X})<0$. Using $(5.2)$, one sees that $\lim _{t \rightarrow+\infty} e^{\operatorname{tad} \tilde{X}} H_{\alpha}^{\prime}=\left(0, \Psi\left(H_{\alpha}\right)\right)$.

Let $\mathfrak{p}_{\tilde{X}}$ be the space of $Y$ in $\mathfrak{g}$ such that $\lim _{t \rightarrow+\infty} e^{\operatorname{tad} \tilde{X}} Y$ exists. Then $\mathfrak{p}_{\tilde{X}}$ is a parabolic subalgebra of $\mathfrak{g}$ with Levi subalgebra $\mathfrak{l}_{\tilde{X}}$ equal to the centralizer of $\tilde{X}$ in $\mathfrak{g}$. The nilradical $\mathfrak{u}_{\tilde{X}}$ of $\mathfrak{p}_{\tilde{X}}$ is equal to the set of $Y \in \mathfrak{g}$ such that $\lim _{t \rightarrow+\infty} e^{\operatorname{tad} \tilde{X}} Y=0$. Hence $H_{\alpha} \in \mathfrak{u}_{\tilde{X}}$ which implies that $H_{\alpha}$ is nilpotent. As $\Psi$ is the differential of a rational character of $\underline{H}$, one has $\Psi\left(H_{\alpha}\right)=0$. 
If $\alpha(\tilde{X})=0$, again, as $X \in \mathfrak{a}_{Z}^{--}$, if $X_{\alpha, \beta} \neq 0$, one has $\beta(\tilde{X})<0$. From (5.2), one deduces that $\lim _{t \rightarrow+\infty} e^{\operatorname{tad} \tilde{X}} H_{\alpha}=X_{-\alpha}$. This shows that $H_{\alpha} \in \mathfrak{p}_{\tilde{X}}$ and $X_{-\alpha} \in \mathfrak{l}_{\tilde{X}}$. Moreover $H_{\alpha}=X_{-\alpha}+X_{\alpha}^{\prime}$ with $X_{\alpha}^{\prime} \in \mathfrak{u}_{\tilde{X}}$. As $X_{-\alpha}$ is nilpotent, this implies that $H_{\alpha}$ is also nilpotent. Hence $\Psi\left(H_{\alpha}\right)=0$ and, as above, we deduce from (5.3) that:

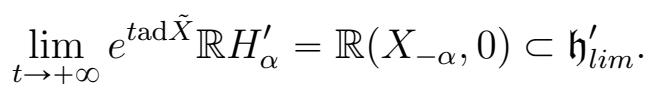

This shows that $\lim _{t \rightarrow+\infty} e^{\operatorname{tad} \tilde{X}} \mathfrak{h}^{\prime}=\mathfrak{h}_{\text {lim }}^{\prime}$ and $X^{\prime} \in \mathfrak{a}_{Z^{\prime}}^{--}$as wanted. This proves that $\mathfrak{a}_{Z^{\prime}}^{--}$ projects onto $\mathfrak{a}_{Z}^{--}$. We get (iii) by taking the closure. Remark that (iii) also follows from $[7$, Corollary 6.10].

To prove (iv), let us assume that $Z$ is wave-front. Let $X^{\prime} \in \mathfrak{a}_{Z^{\prime}}^{-}$and let $X$ be its projection on $\mathfrak{a}_{Z}$. As $Z$ is wave-front, there exists $\tilde{X} \in \mathfrak{a}_{L}^{-}$which projects onto $X$. Hence there exists $r \in \mathbb{R}$ such that $(\tilde{X}, r) \in \mathfrak{a}_{L}^{-} \times \mathbb{R}=\mathfrak{a}_{L^{\prime}}^{-}$projects on $X^{\prime} \in \mathfrak{a}_{Z^{\prime}}$. Hence $Z^{\prime}$ is wave-front.

The last assertion (v) of the Lemma follows from the equalities $\mathfrak{a}_{Z, E}=\mathfrak{a}_{Z}^{-} \cap-\mathfrak{a}_{Z}^{-}$and $\mathfrak{a}_{Z^{\prime}, E}=\mathfrak{a}_{Z^{\prime}}^{-} \cap-\mathfrak{a}_{Z^{\prime}}^{-}$. This achieves to prove the Lemma.

We define $\hat{\mathfrak{h}}^{\prime}=\mathfrak{h}^{\prime}+\mathfrak{a}_{Z^{\prime}, E}=\hat{\mathfrak{h}} \times \mathbb{R}$. Then $\widehat{H}_{0}^{\prime}=\widehat{H}_{0} \times \mathbb{R}^{\times}$, where $\widehat{H}_{0}=\widehat{H}_{\mathbb{C}, 0} \cap G$ and $\hat{H}_{0}^{\prime}=\widehat{H}_{\mathbb{C}, 0}^{\prime} \cap G^{\prime}$. The $\left(P^{\prime}, \hat{H}_{0}^{\prime}\right)$-orbits have representatives in $G$ as $P^{\prime}$ contains $\{1\} \times \mathbb{R}^{\times}$. Then, if $w^{\prime} \in G^{\prime}, P^{\prime} w^{\prime} \hat{H}_{0}^{\prime}=\left(P w \widehat{H}_{0}\right) \times \mathbb{R}^{\times}$for some $w \in G$ and $P^{\prime} w^{\prime} \hat{H}_{0}^{\prime}$ is open in $G^{\prime}$ if and only if $P w \hat{H}_{0}$ is open in $G$. Let us consider the set $\mathcal{F}$ given in the proof of Lemma 1.1. It follows from the previous discussion that the corresponding set for $Z^{\prime}$ can be taken equal to $\mathcal{F}$.

We come to the set $\mathcal{F}^{\prime}$ defined in [11, equation (3.15)], i.e. $\mathcal{F}^{\prime}$ is a set of representatives of the finite group $\hat{H}_{0} / H A_{Z, E}$. Then $\hat{H}_{0}=A_{Z, E} \mathcal{F}^{\prime} H$. As $\hat{H}_{0}^{\prime}=\widehat{H}_{0} \times \mathbb{R}^{\times}$(see above) and $A_{Z^{\prime}, E}=A_{Z, E} \times \mathbb{R}^{+*}$, one has $\widehat{H}_{0}^{\prime}=A_{Z^{\prime}, E}\left(\mathcal{F}^{\prime} \times\{ \pm 1\}\right) H^{\prime}$. Hence the set $\mathcal{F}^{\prime}$ for $Z^{\prime}$ can be taken to be contained in $\mathcal{F}^{\prime} \times\{ \pm 1\}$. Looking at the end of the proof of Lemma 1.1, one sees that one can arrange the set $\mathcal{W}^{\prime}$ given for $Z^{\prime}$ by this Lemma in such a way that $\mathcal{W}$ is contained in $\mathcal{W}^{\prime}$.

Let us recall that we have chosen a section $s: \mathfrak{a}_{Z} \rightarrow \tilde{\mathfrak{a}}_{Z} \subset \mathfrak{a}_{L}$. We may and will choose a section $s^{\prime}: \mathfrak{a}_{Z^{\prime}} \rightarrow \mathfrak{a}_{L^{\prime}}$ such that, if $e_{1} \in \mathfrak{a}_{Z^{\prime}}$ is the image of $(0,1) \in\{0\} \times \mathbb{R}$ in $\mathfrak{a}_{Z^{\prime}}$, then $s^{\prime}\left(e_{1}\right)=(0,1)$. Then it follows easily from Lemma $5.2(\mathrm{v})$ that $\hat{H}_{\mathbb{C}, 0}^{\prime}$ contains $\mathbb{C}^{\times}$and is equal to $\widehat{H}_{\mathbb{C}, 0} \times \mathbb{C}^{\times}$.

Let $I \subset S$ and $X \in \mathfrak{a}_{I}^{--} \subset \mathfrak{a}_{Z}^{-}$. Let $X^{\prime} \in \mathfrak{a}_{Z^{\prime}}^{-}$which projects to $X$. It follows from [11, Section 3.1.2] that there exists $\lim _{t \rightarrow+\infty} e^{\operatorname{tad}\left(X^{\prime}\right)}\left(\mathfrak{h}^{\prime}\right)$ that we will denote by $\mathfrak{h}_{I}^{\prime}$. Let us show that $\mathfrak{h}_{I}^{\prime}=\lim _{t \rightarrow+\infty} e^{\operatorname{tad} X}\left(\mathfrak{h}^{\prime}\right)$. In fact, if $(\tilde{X}, r) \in \mathfrak{a}_{L} \times \mathbb{R}$ projects on $X^{\prime}, e^{\operatorname{tad} X^{\prime}}\left(\mathfrak{h}^{\prime}\right)=e^{\operatorname{tad}(\tilde{X}, r)\left(\mathfrak{h}^{\prime}\right) .}$ As $\{0\} \times \mathbb{R}$ is central in $\mathfrak{g}^{\prime}$, one gets $e^{\operatorname{tad} X^{\prime}}\left(\mathfrak{h}^{\prime}\right)=e^{\operatorname{tad} \tilde{X}}\left(\mathfrak{h}^{\prime}\right)=e^{\operatorname{tad} X}\left(\mathfrak{h}^{\prime}\right)$.

Let $H_{I}^{\prime}$ be the analytic subgroup of $G^{\prime}$ with Lie algebra $\mathfrak{h}_{I}^{\prime}$. Then (cf. loc.cit.) $Z_{I}^{\prime}=G^{\prime} / H_{I}^{\prime}$ is a real spherical space and $\mathfrak{a}_{Z_{I}^{\prime}}=\mathfrak{a}_{Z^{\prime}}$.

5.3 Lemma. Using the notation of (iii) of the previous Lemma, one has that $\mathfrak{a}_{Z_{I}^{\prime}}^{-}$is invariant by the image of $\{0\} \times \mathbb{R}$ in $\mathfrak{a}_{Z^{\prime}}$ and projects onto $\mathfrak{a}_{Z_{I}}^{-}$. 
Proof. The invariance of $\mathfrak{a}_{Z_{I}^{\prime}}^{-}$by the image of $\{0\} \times \mathbb{R}$ in $\mathfrak{a}_{Z^{\prime}}$ is proved in the same way than the invariance of $\mathfrak{a}_{Z^{\prime}}^{-}$(or $\mathfrak{a}_{Z^{\prime}}^{-}$) in the proof of Lemma 5.2(iii).

Let us recall that $e_{1}$ is the image of $(0,1)$ in $\mathfrak{a}_{Z^{\prime}}$. For simplicity, we will identify (not canonically) $\mathfrak{a}_{Z^{\prime}}$ to $\mathfrak{a}_{Z} \times \mathbb{R}$ by choosing a section $\sigma$ of the projection of $\mathfrak{a}_{Z^{\prime}}$ to $\mathfrak{a}_{Z}$ and defining a linear bijection $\mathfrak{a}_{Z} \times \mathbb{R} \rightarrow \mathfrak{a}_{Z^{\prime}}$ by $(X, r) \mapsto r e_{1}+\sigma(X)$. Then Lemma 5.2(iii) can be rewritten as $\mathfrak{a}_{Z^{\prime}}=\mathfrak{a}_{Z} \times \mathbb{R}$. Let $C \subset \mathfrak{a}_{Z}$ (resp. $C^{\prime} \subset \mathfrak{a}_{Z^{\prime}}$ ) be the closed convex cone generated by the set $S$ (resp. $\left.S^{\prime}\right)$ of spherical roots of $Z$ (resp. $\left.Z^{\prime}\right)$. One has $S \subset \mathfrak{a}_{Z}^{*}$ and also $S^{\prime} \subset \mathfrak{a}_{Z}^{*}$ as $\mathbb{R} e_{1} \subset \mathfrak{a}_{Z^{\prime}, E}$.

To finish the proof of Lemma 5.3, we will need the following simple Lemma.

5.4 Lemma. Let $C$ be a convex cone generated in a real vector space $E$ by a finite family $\Xi$ of linearly independent vectors. If $S$ is a finite set of generators o this cone, then $S$ contains a family $S_{0}$ with a bijection $\xi \mapsto \alpha(\xi)$ from $\Xi$ onto $S_{0}$ such that, for all $\xi \in \Xi, \alpha(\xi)$ is a non-zero and proportional to $\Xi$. One says that the elements of $\Xi$ and $S_{0}$ are proportional.

Proof. Let $\Xi=\left\{\xi_{1}, \ldots, \xi_{l}\right\}$ and $S=\left\{\alpha_{1}, \ldots, \alpha_{n}\right\}$. We can assume that $\xi_{1}, \ldots, \xi_{l}$ generate $E$. Let $f_{i}$ be a linear form on $E$ such that:

$$
\begin{aligned}
& f_{i}\left(\xi_{i}\right)=0, \\
& f_{i}\left(\xi_{j}\right)>0 \text { if } j \neq i .
\end{aligned}
$$

Hence $f_{i} \geqslant 0$ on $C$. Let $\xi_{i_{0}} \in \Xi$. As $\xi_{i_{0}} \in C$ and $S$ generates $C$, one can write:

$$
\xi_{i_{0}}=\sum_{j=1}^{n} c_{j} \alpha_{j}
$$

Then $f_{i_{0}}\left(\xi_{i_{0}}\right)=0$ implies that, for all $j$ such that $c_{j} \neq 0$, one has $f_{i_{0}}\left(\alpha_{j}\right)=0$. Let $j \in$ $\{1, \ldots, n\}$ be such that $c_{j} \neq 0$. Let us show that $\alpha_{j}$ is proportional to $\xi_{i_{0}}$. In fact, one can write $\alpha_{j}=\sum_{i=1}^{l} d_{i} \xi_{i}$ with $d_{i} \geqslant 0$. Then $f_{i_{0}}\left(\alpha_{i}\right)=0$ implies that, for $i \neq i_{0}, d_{i}=0$, as $f_{i_{0}}\left(\xi_{i}\right)>0$ for $i \neq i_{0}$. The set of such $\alpha_{i}{ }^{\prime}$ s, when $i_{0}$ varies, is denoted by $S_{0}$. Such a $S_{0}$ has the required properties.

End of proof of Lemma 5.3. From [7, Corollary 12.5], $C$ is the cone generated by $\Xi_{\mathbb{R}}(Z)$ whose elements are linearly independent (cf. loc.cit., Corollary 10.9), and similarly for $C^{\prime}$. Let $S_{0}$ (resp. $S_{0}^{\prime}$ ) be the subset of $S$ (resp. $S^{\prime}$ ) defined by Lemma 5.4 which forms a set of linear independent generators of $C$ (resp. $C^{\prime}$ ).

Note that $C$ (resp. $C^{\prime}$ ) is the dual cone of $\mathfrak{a}_{Z}^{-}$(resp. $\mathfrak{a}_{Z^{\prime}}^{-}$) because $\mathfrak{a}_{Z}^{-}$(resp. $\mathfrak{a}_{Z^{\prime}}^{-}$) is the dual cone of $C$ (resp. $C^{\prime}$ ) and $C$ (resp. $C^{\prime}$ ) is closed. From Lemma 5.2(iii), one sees that $C=C^{\prime}$. Hence, by Lemma 5.4, the elements of $S_{0}$ and $S_{0}^{\prime}$ are proportional.

Now $\mathfrak{a}_{Z_{I}}^{-}=\left\{Y \in \mathfrak{a}_{Z}: \alpha(X) \leqslant 0, \alpha \in I\right\}$. Let $I_{0}=I \cap S_{0}$. Let us prove that $\mathfrak{a}_{Z_{I}}^{-}=$ $\left\{Y \in \mathfrak{a}_{Z}: \alpha(X) \leqslant 0, \alpha \in I_{0}\right\}$. Let us recall that, as $X \in \mathfrak{a}_{I}^{--} \subset \mathfrak{a}_{Z}^{-}$, one has $\alpha(X) \leqslant 0$ for all $\alpha \in S$, and $I=\{\alpha \in S: \alpha(X)=0\}$. Let $\alpha \in I$. Then $\alpha \in C$ and hence is a linear combination of elements of $S_{0}$ with coefficients greater or equal to zero, as $S_{0}$ generates 
the convex cone $C$. Evaluating at $X$, one sees that the only elements of $S_{0}$ which actually contribute to this linear combination are elements of $I_{0}$. Our claim follows. Similarly, one has $I^{\prime}=\left\{\alpha \in S^{\prime}: \alpha\left(X^{\prime}\right)=0\right\}$ and $I_{0}^{\prime}=I^{\prime} \cap S_{0}^{\prime}$.

We have identified the elements of $S^{\prime}$ with elements of $\mathfrak{a}_{Z}^{*}$ and one has $\alpha\left(X^{\prime}\right)$ is equal to $\alpha(X)$ for this identification. Then $I_{0}^{\prime}=\left\{\alpha \in S_{0}^{\prime}: \alpha(X)=0\right\}$. As the elments $S_{0}$ and $S_{0}^{\prime}$ are proportional, the elements of $I_{0}$ and $I_{0}^{\prime}$ are proportional. But, as above, $\mathfrak{a}_{Z_{I}^{\prime}}^{-}=\left\{Y \in \mathfrak{a}_{Z^{\prime}}^{-}\right.$: $\left.\alpha(Y) \leqslant 0, \alpha \in I_{0}^{\prime}\right\}$. Hence one gets the equality:

$$
\mathfrak{a}_{Z_{I}^{\prime}}^{-}=\mathfrak{a}_{Z_{I}}^{-} \times \mathbb{R}
$$

This implies that $\mathfrak{a}_{Z_{I}^{\prime}, E}=\mathfrak{a}_{Z_{I}, E} \times \mathbb{R}$. As for $Z$, one sees that this implies that one can choose $\mathcal{W}_{I}^{\prime}$ (for $Z_{I}^{\prime}$ ) which contains $\mathcal{W}_{I}$.

Starting with $w_{I} \in \mathcal{W}_{I} \subset \mathcal{W}_{I}^{\prime}$, one first find with [11, Lemma 3.9] an element $w^{\prime}$ of $G^{\prime}$ such that $P^{\prime} w_{I} a_{s} H^{\prime}=P^{\prime} w^{\prime} H^{\prime}$ for any $s \geqslant s_{0}$ and such that $P^{\prime} w^{\prime} H^{\prime}$ is open. One can take $w^{\prime} \in G$, as $P^{\prime}$ contains $\{1\} \times \mathbb{R}^{\times}$, and even in $\mathcal{W}$, as $\mathcal{W}$ contains a set of representatives of all open $(P, H)$-double cosets (cf. [11, just after equation (3.15)]). Then the elements $b_{s}, m_{s}, h_{s} \in G$ given by (4.16) for $G$ can be obtained via the natural projection $G^{\prime} \rightarrow G$ from the elements $b_{s}^{\prime}, m_{s}^{\prime}, h_{s}^{\prime} \in G^{\prime}$ given by equation (4.16) for $G^{\prime}$. Hence, if the Proposition 5.1 is true for $Z^{\prime}$, it is true for $Z$.

\subsection{Preliminaries to the proof of Proposition 5.1 when $Z$ is quasi- affine}

A finite dimensional representation of $G$ is said $H$-spherical (resp. $K$-spherical) if it has a non zero $H$-fixed (resp. $K$-fixed) vector. A finite dimensional representation of $G$ is said $H$-semispherical if it has a real line fixed by $H$. Let $\Gamma\left(\operatorname{resp} . \Gamma_{s}, \Gamma_{K}\right)$ be the set of (equivalence classes of) finite dimensional $H$-spherical (resp. $H$-semispherical, $H$ and $K$-spherical) irreducible representations of $G$. If $(\pi, V) \in \Gamma_{s}$, let $\lambda_{\pi} \in \mathfrak{a}^{*}$ be the highest weight of $\pi^{*}$ with respect to $\mathfrak{a}$ and $\mathfrak{n}$. Let us show that any non zero $v \in V$, which transforms under a character of $H$, is not orthogonal to the space of weight $\lambda_{\pi}$ in $V^{*}, V_{\lambda_{\pi}}^{*}$. If it was not the case, denoting by $P_{\mathbb{C}, 0}$ the analytic subgroup of $\underline{G}(\mathbb{C})$ with Lie algebra $\mathfrak{p}_{\mathbb{C}}$, one would conclude:

$$
<\pi(h) v, \pi^{*}(p) v_{\lambda_{\pi}}^{*}>=0, \quad h \in H_{\mathbb{C}, 0}, p \in P_{\mathbb{C}, 0}, v \in V^{H}, v_{\lambda_{\pi}}^{*} \in V_{\lambda_{\pi}}^{*} .
$$

But $H_{\mathbb{C}, 0} P_{\mathbb{C}, 0}$ is Zariski open in $\underline{G}(\mathbb{C})$, hence dense in $\underline{G}(\mathbb{C})$. One would then deduce from the above equality that $v=0$. This proves that for any $v$ as above, there exists $v_{\lambda_{\pi}}^{*} \in V_{\lambda_{\pi}}^{*}$ such that:

$$
<v, v_{\lambda_{\pi}}^{*}>\neq 0 .
$$

If $(\pi, V) \in \Gamma$ and $a \in A \cap H$, on one hand $<\pi(a) v, v_{\lambda_{\pi}}^{*}>=<v, v_{\lambda_{\pi}}^{*}>$, and on the other hand $<\pi(a) v, v_{\lambda_{\pi}}^{*}>=a^{-\lambda_{\pi}}<v, v_{\lambda_{\pi}}^{*}>$. Thus $a^{\lambda_{\pi}}=1$ for any $a \in A \cap H$. This implies that $\lambda_{\pi} \in \mathfrak{a}_{Z}^{*}$. 
5.5 Lemma. Let $(\pi, V) \in \Gamma_{s}$. Let $v$ be a non zero vector of $V$ which transforms under a character $\psi$ of $H$. Let $\lambda$ be the highest weight of $\pi^{*}$ with respect to $\mathfrak{a}$ and $\mathfrak{n}$. Let us decompose $v$ under the weight $\mathfrak{a}$-subspaces of $V$. Then:

$$
v=\sum_{\mu \in \mathbb{N} \Sigma_{\mathfrak{n}}} v_{-\lambda+\mu}
$$

where $v_{-\lambda+\mu}$ is non zero and of weight $-\lambda+\mu, \mu \in \mathfrak{a}_{Z}^{*}$. Moreover, if $X \in \mathfrak{a}_{Z}^{--}$and $\mu$ is non zero, then $\mu(X)<0$.

Proof. On one hand, as one notices, $\left.<v, v_{\lambda}^{*}\right\rangle \neq 0$ for some element $v_{\lambda}^{*}$ of $V_{\lambda}^{*}$. On the other hand, as $\pi(a) v=\psi(a) v$ for $a \in A_{H}$, one has $a^{-\lambda}<v, v_{\lambda}^{*}>=\psi(a)<v, v_{\lambda}^{*}>$ for $a \in A_{H}$. Thus $a^{-\lambda}=\psi(a)$ for all $a \in A_{H}$. Then all $\mu$ in the sum are trivial on $\mathfrak{a}_{H}$ and hence $\mu \in \mathfrak{a}_{Z}^{*}$.

Let $X \in \mathfrak{a}_{Z}^{--}$and $a_{t}=\exp (t X)$. Let $Y \in \mathfrak{n}^{-} \subset \mathfrak{h}_{\text {lim }}$. Then, from (5.1), there exists a sequence $\left(t_{n}\right)$ which tends to $+\infty$ and a sequence $\left(X_{n}\right)$ in $\mathfrak{h}$ such that $Y_{n}=\operatorname{Ad}\left(a_{t_{n}}\right) X_{n}$ tends to $Y$. By extracting a subsequence, and using the conjugacy of the unit sphere, one can find a sequence $\left(c_{n}\right)$ of non zero real numbers such that $\left(v_{n}\right)=\left(c_{n} \pi\left(a_{t_{n}}\right) v\right)$ converges to a non zero limit $w$. Then $\pi\left(Y_{n}\right) v_{n}$ tends to $\pi(Y) w$. But

$$
\begin{aligned}
\pi\left(Y_{n}\right) v_{n} & =c_{n} \pi\left(a_{t_{n}}\right) \pi\left(X_{n}\right) v_{n} \\
& =\Psi\left(X_{n}\right) v_{n}
\end{aligned}
$$

where $\Psi$ is the differential of $\psi$. Hence $\pi(Y) w$ is proportional to $w$. As $\mathfrak{n}^{-}$acts by nilpotent operators in $V$, this implies

$$
\pi(Y) w=0, \quad Y \in \mathfrak{n}^{-} .
$$

Then $w$ is a lowest weight vector. Projecting onto the weight spaces, one sees that, up to a scalar factor, $w=v_{-\lambda}$ and that $\left(c_{n}\right)$ is equivalent to $a_{t_{n}}^{\lambda}$. Hence we may take $c_{n}=a_{t_{n}}^{\lambda}$. As $v_{n}=a_{t_{n}}^{\lambda} \pi\left(a_{t_{n}}\right) v$, one sees that, for all $\mu \neq 0$ occuring in the sum, $e^{\mu\left(t_{n} X\right)}$ tends to zero. Hence our claim follows.

5.6 Proposition. Let $F$ be a non identically zero regular function on $G$ which is left- $N$ invariant, transforms on the left by a character $\chi$ of $A$ and on the right by a real character $\psi$ of $H$. Let $\lambda \in \mathfrak{a}^{*}$ be the differential of $\chi$ at the identity. Let $X_{I} \in \mathfrak{a}_{I}^{--}$and $\tilde{X}_{I} \in \mathfrak{a}_{L}$ which projects onto $X_{I}$. Let $\tilde{a}_{s}=\exp \left(s \tilde{X}_{I}\right)$. Then

(i) $\lim _{s \rightarrow+\infty} a_{s}^{\lambda} R\left(a_{s}\right) F$ exists in $\mathbb{C}[G]$ and is non zero, where $R$ is the right regular representation of $G$ in $\mathbb{C}[G]$, the space of complex valued regular functions on $G$. We denote it by $F_{I}$.

(ii) The function $F_{I}$ is left-N-invariant and transforms by the character $\chi$ of $A$ on the left and by a real character $\psi_{I}$ of $H_{I}$ on the right. Moreover, if $\psi$ is trivial, then $\psi_{I}$ is trivial too.

(iii) One has $F_{\mid L_{\mathbb{C}, 0}}=F_{I \mid L_{\mathbb{C}, 0}}$. 
Proof. Let $\Psi$ be the differential of $\psi$ at the identity. Let $V_{F}$ or $V$ be the linear span in $\mathbb{C}[G]$ of the right translates of $F$ by elements of $G$. Let $v^{*}$ be the linear form on $V$ given by the evaluation at 1 . Let $\pi$ be the right regular representation of $G$ in $V$. Then one has

$$
F(g)=<v^{*}, \pi(g) F>, \quad g \in G .
$$

Our hypothesis on $F$ implies that $(\pi, V) \in \Gamma_{s}$ and $v^{*}$ is of weight $\lambda$ under $\mathfrak{a}$ for the contragredient representation $\pi^{*}$ of $\pi$, and is left- $N$-invariant. Obviously $v^{*}$ is cyclic for $\pi^{*}$. The decomposition $U(\mathfrak{g})=U\left(\mathfrak{n}^{-}\right) U(\mathfrak{a}) U(\mathfrak{m}) U(\mathfrak{n})$ implies that the $\mathfrak{a}$-weights of $V^{*}$ are of the form $\lambda$ plus a sum of roots of $\mathfrak{a}$ in $\mathfrak{n}^{-}$. Then the weights of $V$ are of the form $-\lambda$ plus a sum of roots of $\mathfrak{a}$ in $\mathfrak{n}$. One writes $F \in V$ as a sum

$$
F=\sum_{\mu \in \Lambda \subset \mathbb{N} \Sigma_{\mathfrak{n}}} v_{-\lambda+\mu}
$$

where $v_{-\lambda+\mu}$ is a vector of weight $-\lambda+\mu$ in $V$. From Lemma 5.5, one sees that, if $v_{-\lambda+\mu} \neq 0$, one has $\mu\left(X_{I}\right) \leqslant 0$. Then one sees easily that $F_{I}$ exists and satisfies:

$$
F_{I}=\sum_{\mu \in \Lambda, \mu\left(X_{I}\right)=0} v_{-\lambda+\mu}
$$

Let us show that $v_{-\lambda} \neq 0$. In fact, as $P_{\mathbb{C}, 0} H_{\mathbb{C}, 0}$ is Zariski dense in $\underline{G}(\mathbb{C}), F$ is not identically zero on $P_{\mathbb{C}, 0} H_{\mathbb{C}, 0}$. Let $g=n a m h \in P_{\mathbb{C}, 0} H_{\mathbb{C}, 0}$ with $F(g) \neq 0$. Then $<v^{*}, \pi(m) F>\neq 0$. But $<v^{*}, \pi(m) F>=<v^{*}, \pi(m) v_{-\lambda}>$, as weight spaces of $V$ for $\mathfrak{a}$ are $M$-invariant and $v^{*}$ is of weight $\lambda$ and thus orthogonal to $\pi(m) v_{-\lambda+\mu}$ for $\mu \neq 0$. Hence $v_{-\lambda}$ is non zero.

From its definition as a limit, one sees that $F_{I}$ transforms on the left by $\chi$ under the action of $A$ and is left- $N$-invariant. It remains to prove that $F_{I}$ transforms on the right by a real character of $H_{I}$. It is enough to prove that $\mathbb{R} F_{I}$ is right invariant by the action of $\mathfrak{h}_{I}$. It is clear, from the definition of $F_{I}$ as a limit and from the fact that $\mathfrak{l} \cap \mathfrak{h}$ centralizes $\mathfrak{a}$ and $\mathbb{R} F$ is right $\mathfrak{h}$-invariant that $\mathbb{R} F_{I}$ is right invariant by $\mathfrak{l} \cap \mathfrak{h}$. Let $X \in \mathfrak{h}_{I}$ be of the form $X=Y+T_{I}(Y)$, where $Y \in \mathfrak{g}^{-\alpha}$ with $\alpha \in \Sigma_{\mathfrak{u}}$. One has $X^{\prime}=Y+T(Y) \in \mathfrak{h}$ and

$$
X=\lim _{s \rightarrow+\infty} \tilde{a}_{s}^{\alpha} \operatorname{Ad}\left(\tilde{a}_{s}\right) X^{\prime}
$$

Hence

$$
\tilde{a}_{s}^{\lambda+\alpha} \pi\left(\tilde{a}_{s}\right) \pi\left(X^{\prime}\right) F=\Psi\left(X^{\prime}\right) \tilde{a}_{s}^{\alpha}\left(\tilde{a}_{s}^{\lambda} \pi\left(\tilde{a}_{s}\right) F\right) .
$$

As $\tilde{a}_{s}^{\lambda+\alpha} \pi\left(\tilde{a}_{s}\right) \pi\left(X^{\prime}\right) F=\tilde{a}_{s}^{\alpha} \pi\left(\operatorname{Ad}\left(\tilde{a}_{s}\right) X^{\prime}\right) \tilde{a}_{s}^{\lambda} \pi\left(\tilde{a}_{s}\right) F,(5.5)$ and the definition of $F_{I}$ imply that the left hand side of (5.6) tends to $\pi(X) F_{I}$. The right hand side has the same limit and, as $\left(\tilde{a}_{s}^{\lambda} \pi\left(\tilde{a}_{s}\right) F\right)$ tends to $F_{I}$ and as $\Psi\left(X^{\prime}\right) \tilde{a}_{s}^{\alpha}$ is real, this limit is a real multiple of $F_{I}$. Hence we get

$$
\pi(X) F_{I} \in \mathbb{R} F_{I} .
$$

Thus we have proved that $\mathbb{R} F_{I}$ is invariant by $\pi\left(\mathfrak{h}_{I}\right)$. Hence $F_{I}$ transforms by a real character $\psi_{I}$ of $H_{I}$. 
The proof above shows that, if $\psi$ is trivial, $\psi_{I}$ is also trivial which gives the last statement of (ii).

Let us prove (iii). It is clear that, if $l \in L_{\mathbb{C}, 0}$, as $\tilde{a}_{s} \in A_{L}$,

$$
R\left(\tilde{a}_{s}\right) F(l)=F\left(l \tilde{a}_{s}\right)=\tilde{a}_{s}^{-\lambda} F(l) .
$$

Hence $\tilde{a}_{s}^{\lambda}\left(R\left(\tilde{a}_{s}\right) F\right)(l)=F(l)$. This implies (iii).

We come back to the notation of (4.14), (4.15) and (4.16). Let $F$ be as in Proposition 5.6, i.e. $F$ is a regular function on $G$ which transforms on the left trivially under $N$, by a character $\chi$ of $A$, and which transforms on the right by a real character $\psi$ of $H$. Let $\lambda$ be the differential of $\chi$. Then one has, with the notation of the proof of Proposition 5.6,

$$
\pi\left(\tilde{t}_{I} h_{I} \tilde{a}_{s}\right) F=\psi\left(h_{s}\right) \pi\left(u_{s} m_{s} \tilde{b}_{s} w\right) F .
$$

We define

$$
\begin{aligned}
& F_{s}=\tilde{a}_{s}^{\lambda} \pi\left(\tilde{a}_{s}\right) F, \quad \tilde{F}_{s}=\tilde{a}_{s}^{\lambda} \pi\left(\tilde{b}_{s}\right) F \\
& y_{s}=u_{s} m_{s} \tilde{t}
\end{aligned}
$$

Then, as $\tilde{t}$ and $\tilde{b}_{s}$ commute as elements of $A_{L, \mathbb{C}, 0},(5.7)$ can be rewritten as follows

$$
\pi\left(\tilde{t}_{I} h_{I}\right) F_{s}=\psi\left(h_{s}\right) \psi(h) \pi\left(y_{s}\right) \tilde{F}_{s} .
$$

Let $\mathbf{P}\left(V_{F}\right)$ (resp. $\mathbf{P}_{\mathbb{R}}\left(V_{F}\right)$ ) be the complex projective (resp. real projective) space of $V_{F}$.

\subsection{Lemma. One has}

(i)

$$
F_{s} \underset{s \rightarrow+\infty}{\stackrel{r a p i d}{\longrightarrow}} F_{I}
$$

(ii)

$$
\left[\psi(h) \pi\left(y_{s}\right) \tilde{F}_{s}\right] \underset{s \rightarrow+\infty}{\stackrel{\text { rapid }}{\longrightarrow}}\left[\psi_{I}\left(h_{I}\right) \pi\left(\tilde{t}_{I}\right) F_{I}\right] \quad \text { in } \mathbf{P}_{\mathbb{R}}\left(V_{F}\right)
$$

(iii) Moreover, if $\psi$ is trivial, then $\pi\left(y_{s}\right) \widetilde{F}_{s} \underset{s \rightarrow+\infty}{\stackrel{\text { rapid }}{\longrightarrow}} \pi\left(\tilde{t}_{I}\right) F_{I}$.

Proof. One has with the notation of (5.4),

$$
\begin{aligned}
\left\|F_{s}-F_{I}\right\| & =\left\|\tilde{a}_{s}^{\lambda} \pi\left(\tilde{a}_{s}\right) F-F_{I}\right\| \\
& =\left\|\sum_{\mu \in \Lambda, \mu\left(X_{I}\right) \neq 0} \tilde{a}_{s}^{\mu} v_{-\lambda+\mu}\right\| .
\end{aligned}
$$

But, as $X_{I} \in \mathfrak{a}_{I}^{--}$, one has, from Lemma 5.5, $\mu\left(X_{I}\right)<0$ if $\mu\left(X_{I}\right) \neq 0$. This implies that $\left(F_{s}\right)$ tends rapidly to $F_{I}$ when $s$ goes to $+\infty$. Then, together with (5.9), this implies that $\left[\pi\left(y_{s}\right) \widetilde{F}_{s}\right]=\left[\psi(h) \pi\left(y_{s}\right) \widetilde{F}_{s}\right]$ converges rapidly to $\left[\pi\left(\tilde{t}_{I} h_{I}\right) F_{I}\right]=\left[\psi_{I}\left(h_{I}\right) \pi\left(\tilde{t}_{I}\right) F_{I}\right]$ in $\mathbf{P}_{\mathbb{R}}\left(V_{F}\right)$. If $\psi$ is trivial, one even has $\pi\left(y_{s}\right) \tilde{F}_{s} \underset{s \rightarrow+\infty}{\stackrel{\text { rapid }}{\longrightarrow}} \pi\left(\tilde{t}_{I}\right) F_{I}$. 
5.8 Lemma. Let us recall that $Z$ is quasi-affine. Then

(i) For all $(\pi, V) \in \Gamma_{K}$,

$$
\left(a_{s} b_{s}^{-1}\right)^{\lambda_{\pi}} \underset{s \rightarrow+\infty}{\stackrel{\text { rapid }}{\longrightarrow}} 1
$$

(ii) The family $\left(a_{s} b_{s}^{-1}\right)$ converges rapidly to 1 when $s \rightarrow+\infty$.

Proof. Let $(\pi, V) \in \Gamma_{K}, v_{H}$ be a non zero $H$-fixed vector and let $v^{*} \in V^{*}$ be a non zero highest weight vector with weight $\lambda_{\pi}$. Let $F(g)=<v^{*}, \pi(g) v_{H}>, g \in G$. Then $F$ satisfies the hypothesis of Proposition 5.6 and is even left- $M$-invariant. From Lemma 5.7(iii), one sees in particular that

$$
\left(R\left(y_{s}\right) \widetilde{F}_{s}\right)(1) \underset{s \rightarrow+\infty}{\stackrel{\text { rapid }}{\longrightarrow}}\left(R\left(\tilde{t}_{I}\right) F_{I}\right)(1)
$$

But $y_{s}=u_{s} m_{s} \tilde{t}$. From the definition of $\widetilde{F}_{s}$, one gets

$$
\widetilde{F}_{s}\left(y_{s}\right)=\tilde{a}_{s}^{\lambda_{\pi}} F\left(u_{s} m_{s} \tilde{t} \tilde{b}_{s}\right)
$$

and from the covariance properties of $F$, one sees that

$$
\widetilde{F}_{s}\left(y_{s}\right)=\left(a_{s} b_{s}^{-1}\right)^{\lambda_{\pi}} \tilde{t}^{-\lambda_{\pi}} F(1) .
$$

Then, from (5.10), one deduces that:

$$
\left(a_{s} b_{s}^{-1}\right)^{\lambda_{\pi}} \tilde{t}^{-\lambda_{\pi}} F(1) \underset{s \rightarrow+\infty}{\stackrel{\text { rapid }}{\longrightarrow}} F_{I}\left(\tilde{t}_{I}\right) .
$$

From Proposition 5.6(iii), one has $F_{I}\left(\tilde{t}_{I}\right)=F\left(\tilde{t}_{I}\right)$. But $\tilde{t}_{I} \in \exp \left(i \mathfrak{a}_{L}\right)$, and hence $F\left(\tilde{t}_{I}\right)=$ $\left(\tilde{t}_{I}\right)^{-\lambda_{\pi}} F(1)$. Moreover $F(1) \neq 0$ (see above). This proves that there exist $\varepsilon>0$ and $C>0$ such that, for $s$ large enough,

$$
\left|\left(a_{s} b_{s}^{-1}\right)^{\lambda_{\pi}}-\left(\tilde{t} \tilde{t}_{I}^{-1}\right)^{\lambda_{\pi}}\right| \leqslant C e^{-\varepsilon s}
$$

But $\left(a_{s} b_{s}^{-1}\right)^{\lambda_{\pi}}$ is a positive real number as $\lambda_{\pi} \in \mathfrak{a}^{*}$ and $a_{s} b_{s}^{-1} \in A_{Z}$, and $\left(\tilde{t} \tilde{t}_{I}^{-1}\right)^{\lambda_{\pi}}$ is of modulus one. This implies that $\left(\tilde{t} \tilde{t}_{I}^{-1}\right)^{\lambda_{\pi}}$ is a real number of modulus one, greater or equal than zero, as it is the limit of positive numbers. Then this implies (i).

The assertion (ii) is a consequence of (i) and of the fact that $\left\{\lambda_{\pi}: \pi \in \Gamma_{K}\right\}$ generates $\mathfrak{a}_{Z}^{*}$, as $Z$ is quasi-affine (cf. [10, Lemma 3.4]).

5.9 Lemma. With the notation of Lemma 5.7, one has

(i) $\left[\psi(h) \pi\left(y_{s}\right) F_{I}\right] \underset{s \rightarrow+\infty}{\stackrel{\mathrm{rapid}}{\longrightarrow}}\left[\psi_{I}\left(h_{I}\right) \pi\left(\tilde{t}_{I}\right) F_{I}\right]$ in $\mathbf{P}_{\mathbb{R}}\left(V_{F}\right)$;

(ii) $\left[\pi\left(y_{s}\right) F_{I}\right] \underset{s \rightarrow+\infty}{\stackrel{\text { rapid }}{\longrightarrow}}\left[\pi\left(\tilde{t}_{I}\right) F_{I}\right]$ in $\mathbf{P}\left(V_{F}\right)$. 
Proof. Clearly the assertion (ii) follows from (i). Let us prove (i).

For $s \geqslant s_{0}, a_{s} b_{s}^{-1} \in A_{Z}$ and $a_{s} b_{s}^{-1}=\exp X_{s}$ for $X_{s} \in \mathfrak{a}_{Z}$. Hence, as $\exp _{\mid \mathfrak{a}_{Z}}$ is a diffeomorphism, $X_{s} \underset{s \rightarrow+\infty}{\stackrel{\text { rapid }}{\longrightarrow}} 0$ and, as the section defined in (1.3) is linear, one has:

$$
\tilde{X}_{s} \underset{s \rightarrow+\infty}{\stackrel{\text { rapid }}{\longrightarrow}} 0 .
$$

We use the notation of (5.7) and (5.8). Then

$$
\begin{aligned}
\left\|\tilde{F}_{s}-F_{I}\right\| & =\left\|\pi\left(\exp \left(-\tilde{X}_{s}\right)\right) F_{s}-F_{I}\right\| \\
& \leqslant\left\|\pi\left(\exp \left(-\tilde{X}_{s}\right)\right)\left(F_{s}-F_{I}\right)\right\|+\left\|\left(\pi\left(\exp \left(-\tilde{X}_{s}\right)\right)-\mathrm{Id}\right) F_{I}\right\| .
\end{aligned}
$$

Let us show that $\left\|\left(\pi\left(\exp \left(-\tilde{X}_{s}\right)\right)-\mathrm{Id}\right) F_{I}\right\|$ tends rapidly to zero. It suffices to decompose $F_{I}$ into eigenvectors for $A_{L}$ and to use that, if $\lambda \in \mathfrak{a}_{L}^{*}$, then, for $s$ large enough, $\left(e^{-\lambda\left(\tilde{X}_{s}\right)}-1\right)$ is equivalent to $-\lambda\left(\tilde{X}_{s}\right)$. Then, from Lemma 5.7(i) and (5.11), it follows that

$$
\widetilde{F}_{s} \underset{s \rightarrow+\infty}{\stackrel{\text { rapid }}{\longrightarrow}} F_{I} .
$$

One knows that $\left(u_{s}\right)$ converges to 1 and $\left(m_{s}\right)$ converges to $m_{w_{I}}$. Then $\left(y_{s}\right)$ lies in a compact set. Hence $\pi\left(y_{s}\right)\left(\widetilde{F}_{s}-F_{I}\right)$ converges rapidly to zero as $\widetilde{F}_{s}$ converges rapidly to $F_{I}$. But, from Lemma 5.7(ii),

$$
\left[\psi(h) \pi\left(y_{s}\right) \widetilde{F}_{s}\right] \underset{s \rightarrow+\infty}{\stackrel{\mathrm{rapid}}{\longrightarrow}}\left[\psi_{I}\left(h_{I}\right) \pi\left(\tilde{t}_{I}\right) F_{I}\right] \text { in } \mathbf{P}_{\mathbb{R}}\left(V_{F}\right) .
$$

As $\left(y_{s}\right)$ converges, $\pi\left(y_{s}\right) F_{I}$ has a non zero limit and one can apply Lemma B.7 to $v_{s}=$ $\psi(h) \pi\left(y_{s}\right) F_{I}$ and $w_{s}=\psi(h) \pi\left(y_{s}\right) \widetilde{F}_{s}$. One concludes that:

$$
\left[\psi(h) \pi\left(y_{s}\right) F_{I}\right] \underset{s \rightarrow+\infty}{\stackrel{\mathrm{rapid}}{\longrightarrow}}\left[\psi_{I}\left(h_{I}\right) \pi\left(\tilde{t}_{I}\right) F_{I}\right] \text { in } \mathbf{P}_{\mathbb{R}}\left(V_{F}\right) .
$$

\subsection{End of proof of Proposition 5.1 when $Z$ is quasi-affine}

We will now refine our choice of $L$ in Section 1. We will use some results of [8].

With the notation of Definition 3.5 of loc.cit., one can find a real regular function on $G, f \in \mathcal{P}_{++}$, such that $H_{f}^{\times}=J$ (cf. Lemma 3.11 in loc.cit. for the notation). In particular (cf. loc.cit. equation (3.1)), $f$ transforms on the left (resp. the right) by a real character $\chi$ of $P$ (resp. $\psi$ of $H$ ). Moreover, from the definition, one has $H \subset H_{f}^{\times}$.

Let $V_{f}$ be the linear span of the right translates $R(g) f$ of $f$ by the elements $g \in G$ and let $\pi$ be the right regular representation of $G$ on $V_{f}$. Let $\widehat{Z}_{f}$ be the closure of $\operatorname{span}\{[R(g) f]: g \in G\}$ in the complex projective space $\mathbf{P}\left(V_{f}\right)$.

With loc.cit., Proposition 3.18, we get a parabolic subgroup $Q$ which is $G / H_{f}^{\times}$-adapted to $P$ and a Levi subgroup $L$ of $Q$ such that $L \cap H_{f}^{\times}=Q \cap H_{f}^{\times}$. As $H \subset H_{f}^{\times}$, one also has $L \cap H=Q \cap H$. This is our choice of $L$. 
With the notation of this proposition, its proof shows that $S_{Y}$ is closed in $\hat{Z}_{0}$, as $S_{Y}=$ $\mu^{-1}\{\mu(\hat{z})\}$. We consider, with the notation of Proposition 5.6, $\left[f_{I}\right]$, which is obtained as a limit in $\mathbf{P}_{\mathbb{R}}\left(V_{f}\right)$ of right translates of $[f]$ by elements of $L$. As $S_{Y}$ is $L$-invariant, one deduces that this limit is in $S_{Y}$. It follows from [8, Proposition 3.18(2)], that the stabilizer $Q_{f_{I}}^{\times}$of $\left[f_{I}\right]$ in $Q$ is contained in $L$. Let us denote it by $L_{f_{I}}^{\times}$. As $f$ is real on $G, f_{I}$ is also real and the Lie algebra of the stabilizer $\left(Q_{\mathbb{C}, 0}\right)_{f_{I}}^{\times}$of $\left[f_{I}\right]$ in $Q_{\mathbb{C}, 0}$ is equal to the complexified Lie algebra of $Q_{f_{I}}^{\times}=L_{f_{I}}^{\times}$. Hence

$$
\left[\left(Q_{\mathbb{C}, 0}\right)_{f_{I}}^{\times}\right]_{0}=\left(L_{f_{I}}^{\times}\right)_{\mathbb{C}, 0}
$$

We apply Lemma 5.9(ii) to $F=f$. The convergence in Lemma 5.9(ii) is a convergence in $\left[\pi\left(Q_{\mathbb{C}, 0}\right) f_{I}\right]=Q_{\mathbb{C}, 0} /\left(Q_{\mathbb{C}, 0}\right)_{f_{I}}^{\times}$. Hence, by Lemma B.6, one also has that $y_{s}\left[\left(Q_{\mathbb{C}, 0}\right)_{f_{I}}^{\times}\right]_{0}$ converges rapidly in $Q_{\mathbb{C}, 0} /\left[\left(Q_{\mathbb{C}, 0}\right)_{f_{I}}^{\times}\right]_{0}$. But (cf. $(5.12)$ )

$$
Q_{\mathbb{C}, 0} /\left[\left(Q_{\mathbb{C}, 0}\right)_{f_{I}}^{\times}\right]_{0}=U \times\left(L_{\mathbb{C}, 0} /\left(L_{f_{I}}^{\times}\right)_{\mathbb{C}, 0}\right) .
$$

Hence, as $y_{s}=\tilde{t}\left(\tilde{t}^{-1} u_{s} \tilde{t}\right) m_{s}$, one sees that $\left(\tilde{t}^{-1} u_{s} \tilde{t}, m_{s}\left(L_{f_{I}}^{\times}\right)_{\mathbb{C}, 0}\right)$ converges rapidly in $U \times$ $\left(L_{\mathbb{C}, 0} /\left(L_{f_{I}}^{\times}\right)_{\mathbb{C}, 0}\right)$ to $\left(1, m_{w_{I}}\left(L_{f_{I}}^{\times}\right)_{\mathbb{C}, 0}\right)$. It follows in particular that $\left(\tilde{t}^{-1} u_{s} \tilde{t}\right)$ converges rapidly to 1. Hence

$$
u_{s} \underset{s \rightarrow+\infty}{\stackrel{\text { rapid }}{\longrightarrow}} 1
$$

One also gets that

$$
m_{s}\left(L_{f_{I}}^{\times}\right)_{\mathbb{C}, 0} \underset{s \rightarrow+\infty}{\stackrel{\text { rapid }}{\longrightarrow}} m_{w_{I}}\left(L_{f_{I}}^{\times}\right)_{\mathbb{C}, 0} .
$$

5.10 Lemma. Let $f$ be a real regular function on $U \times(L / L \cap H)$, that we identify with an open subset of $Z$, which is left-U-invariant and which transforms on the left by a character $\chi_{f}$ of $A$.

Then there exists a real regular function $h_{f}$ on $Z$, positive valued, which is not identically zero on $L / L \cap H$, left- $N$-invariant, which transforms under a character $\chi_{h_{f}}$ by the left action of $A$, and such that $F_{f}=h_{f} f$ is regular on $Z$.

Proof. This is similar to the proof of [10, Lemma 3.4]. We give it for sake of completeness.

From the definition of the rational function (cf. [3, AG.8.1]), $f$ is a rational function on $Z$. As $Z$ is quasi-affine, $Z$ is an open set in an affine set $\bar{Z}$. Then the field of rational functions on $Z, \mathbb{C}(Z)$, is equal to the field of rational functions on $\bar{Z}, \mathbb{C}(\bar{Z})$, which is the field of fractions of $\mathbb{C}[\bar{Z}]$.

Hence there are regular functions $h_{1}, h_{2}$ on $\bar{Z}$ with $f=h_{1} / h_{2}$.

Let $I=\{h \in \mathbb{C}[Z]: h f \in \mathbb{C}[Z]\}$. Then $I \neq\{0\}$ as $h_{2} \in I$, and $I=\bar{I}$ as $f$ is real. Recall that $N=U\left(L_{n} \cap N\right)$ and $L_{n}$ is normal in $L \cap H$. As $f$ is left- $U$-invariant, right- $L \cap H$ invariant and transforms by a character of $A$ on the left, $f$ transforms by a character of $A N$ on the left. Hence $I$ is left- $A N$-invariant. The action of $A N$ on $\mathbb{C}[Z]$ is algebraic, hence locally finite. Thus we can find an element $0 \neq h \in I$ which is an eigenvector for $A N$. One takes $h_{f}=h \bar{h}$.

As $N$ is unipotent, $h$ is $N$-invariant. Moreover $U(L / L \cap H)$ is Zariski dense in $Z$. Then one sees that $h$ is not identically zero on $L / L \cap H$. 
Diagonalizing the action of $A$ on $\mathbb{R}[L / L \cap H]$, one gets a basis $\left(f_{k}\right)_{k \in \mathcal{K}}$ of $\mathbb{R}[L / L \cap H]$, made of functions $f_{k}$ which transform under a (real) character of $A$ by the left regular representation. We extend $f_{k}$ to a left- $U$-invariant function on $U \times L / L \cap H$, still denoted $f_{k}$.

We set, with the notation of Lemma 5.10, $h_{k}=h_{f_{k}}$ and $F_{k}=F_{f_{k}}:=h_{k} f_{k}$. As the real characters of a connected compact Lie group are trivial and as $F_{k}$ is real, one has

$$
\left(M \cap L_{F_{k, I}}^{\times}\right)_{0} \subset M \cap L_{F_{k, I}},
$$

where $L_{F_{k, I}}$ is the stabilizer for the right action of $F_{k, I \mid L}$ in $L$.

For reason of dimension, there exists a finite set $\mathcal{L} \subset \mathcal{K}$ such that the intersection of the Lie algebras of the groups $\left(M \cap L_{F_{k, I}}\right)_{0}, k \in \mathcal{K}$, is equal to the intersection of the Lie algebras of $M \cap L_{F_{k, I}}, k \in \mathcal{L}$.

5.11 Lemma. Let $M_{\mathcal{L}}=\bigcap_{k \in \mathcal{L}} M \cap L_{F_{k, I}}$. Then $M_{\mathcal{L}, 0}$ is contained in $M \cap H$.

Proof of Lemma 5.11. The group $M_{\mathcal{L}, 0}$ is a connected compact Lie group which is generated by its compact one parameter subgroups. Let $S$ be such a one parameter subgroup. Then, by definition of $\mathcal{K}$ and $\mathcal{L}$, one has $S \subset L_{F_{k, I}}$ for all $k \in \mathcal{K}$. Hence in particular, for all $l \in L$, $k \in \mathcal{K}$ and $s \in S$, as $F_{k, I}=F_{k}$ on $L$ (see Proposition 5.6(iii)),

$$
F_{k}(l s)=F_{k}(l)
$$

i.e.

$$
h_{k}(l s) f_{k}(l s)=h_{k}(l) f_{k}(l) .
$$

5.12 Lemma. Let $p, p^{\prime}$ be two non identically zero trigonometric polynomials such that $p p^{\prime}$ is constant. If $p$ is real, then $p$ and $p^{\prime}$ are constant.

Proof. Write $p=e^{i n \theta}\left(a_{0}+\cdots+a_{k} e^{i k \theta}\right)$ with $k \geqslant 0, a_{0} \neq 0, a_{k} \neq 0$, and $p^{\prime}=e^{i n^{\prime} \theta}\left(a_{0}^{\prime}+\cdots+\right.$ $\left.a_{k}^{\prime} e^{i k^{\prime} \theta}\right)$ with $k^{\prime} \geqslant 0, a_{0}^{\prime} \neq 0, a_{k}^{\prime} \neq 0$. Then the $\left(n+n^{\prime}\right)$-th Fourier coefficient of $p p^{\prime}$ is $a_{0} a_{0}^{\prime}$. Moreover its $\left(n+n^{\prime}+k+k^{\prime}\right)$-th Fourier coefficient is non zero. Hence, as $p p^{\prime}$ is constant, $n+n^{\prime}=0, n+n^{\prime}+k+k^{\prime}=0$ and thus $k=k^{\prime}=0$ which implies that $p=a_{0} e^{i n \theta}$. As $p$ is real, one has $n=0$ and hence $n^{\prime}=0$ and $p^{\prime}$ is also constant.

Applying this Lemma, one sees that, for $k$ fixed, if $h_{k}(l) \neq 0$ and $f_{k}(l) \neq 0$, then $f_{k}(l s)=f_{k}(l)$ for all $s \in S$. But the set of $l \in L$ such that $f_{k}(l) \neq 0$ and $h_{k}(l) \neq 0$ is dense in $L$, as $f_{k}$ and $h_{k}$ are not identically zero on $L$ (cf. Lemma 5.10). By continuity, we get for all $k \in \mathcal{K}$,

$$
f_{k}(l s)=f_{k}(l), \quad l \in L, s \in S .
$$

In particular $f_{k}(s)=f_{k}(1)$. But the $f_{k}$ 's separate the points of $L / L \cap H$. Indeed, as $G / H$ is quasi-affine, the regular functions on $G / H$ separate the points of $G / H$ (by restriction of functions on the affine subset in which $G / H$ is open). Then, again by restriction, we get our claim. 
Hence one has $s \in L \cap H$. This proves that $S \subset M \cap H$ which achieves the proof of the Lemma.

Now we consider $V_{k}=V_{F_{k}}$, i.e. the complex linear span of the right translates of $F_{k}$ by elements of $G$ (cf. notation of the proof of Proposition 5.6) and let $\pi_{k}$ be the right regular representation of $G$ on $V_{k}$. One uses Lemma 5.9(i), with $\psi$ trivial, for the $F_{k}$ 's, $k \in \mathcal{L}$, and gets

$$
\left[\pi_{k}\left(y_{s}\right) F_{k, I}\right] \underset{s \rightarrow+\infty}{\stackrel{\mathrm{rapid}}{\longrightarrow}}\left[\pi_{k}\left(\tilde{t}_{I}\right) F_{k, I}\right] \text { in } \mathbf{P}_{\mathbb{R}}\left(V_{k}\right) .
$$

As $\left(u_{s}\right)$ converges rapidly to 1 , one gets:

$$
\left[\pi_{k}\left(m_{s}\right) \pi(\tilde{t}) F_{k, I}\right] \underset{s \rightarrow+\infty}{\stackrel{\mathrm{rapid}}{\longrightarrow}}\left[\pi_{k}\left(\tilde{t}_{I}\right) F_{k, I}\right] \text { in } \mathbf{P}_{\mathbb{R}}\left(V_{k}\right)
$$

and the same is true if one restricts functions to $L$. But $\tilde{t}$ and $\tilde{t}_{I}$ are elements of $\exp \left(i \tilde{\mathfrak{a}}_{Z}\right) \subset$ $A_{\mathbb{C}}$, which is central in $L_{\mathbb{C}, 0}$, and $F_{I}$ transforms on the left by a character $\chi_{k}$ of $A$. Hence $\pi_{k}(\tilde{t}) F_{k, I}=\chi_{k}(\tilde{t}) F_{k, I}$ and $\pi_{k}\left(\tilde{t}_{I}\right) F_{k, I}=\chi_{k}\left(\tilde{t}_{I}\right) F_{k, I}$.

But, if a family of vectors $\left(v_{s}\right)$ in a finite dimensional complex vector space $E$ is such that $\left(\left[v_{s}\right]\right)$ converges rapidly to $[v]$ in $\mathbf{P}_{\mathbb{R}}(E)$ and $z \in \mathbb{C}^{*}$, then $\left(\left[z v_{s}\right]\right)$ converges rapidly to $[z v]$ in $\mathbf{P}_{\mathbb{R}}(E)$. Thus

$$
\left[\pi_{k}\left(m_{s}\right) F_{k, I \mid L}\right] \text { converges rapidly in } \mathbf{P}_{\mathbb{R}}\left(V_{k, \mid L}\right)
$$

where $V_{k, \mid L}$ denotes the space of restrictions to $L$ of elements of $V_{k}$. As $\left(m_{s}\right)$ converges to $m_{w_{I}}$, one then has

$$
\left[\pi_{k}\left(m_{s}\right) F_{k, I \mid L}\right] \underset{s \rightarrow+\infty}{\stackrel{\mathrm{rapid}}{\longrightarrow}}\left[\pi_{k}\left(m_{w_{I}}\right) F_{k, I \mid L}\right] \text { in } \mathbf{P}_{\mathbb{R}}\left(V_{k, \mid L}\right)
$$

Set $V=\oplus_{k \in \mathcal{L}} V_{k}, F=\oplus_{k \in \mathcal{L}} F_{k}, F_{I}=\oplus_{k \in \mathcal{L}} F_{k, I}$ and let $\pi$ be the direct sum of the right regular representations $\pi_{k}$ of $G$ in the $V_{k}$ 's. Then one gets from (5.13) that

$$
\left[\pi\left(m_{s}\right) F_{I \mid L}\right] \underset{s \rightarrow+\infty}{\stackrel{r a p i d}{\longrightarrow}}\left[\pi\left(m_{w_{I}}\right) F_{I \mid L}\right] \text { in } \prod_{k \in \mathcal{L}} \mathbf{P}_{\mathbb{R}}\left(V_{k, \mid L}\right)
$$

Here, if $v=\left(v_{k}\right)_{k \in \mathcal{L}} \in V$, then $[v]=\left(\left[v_{k}\right]\right)_{k \in \mathcal{L}} \in \Pi_{k \in \mathcal{L}} \mathbf{P}_{\mathbb{R}}\left(V_{k}\right)$.

For any $k \in \mathcal{L}$, recall that the stabilizer for the right action of $F_{k, I \mid L}$ in $L$ has been denoted $L_{F_{k, I}}$. Hence, from Lemma B.5, one sees that:

$$
m_{s} M_{\mathcal{L}} \underset{s \rightarrow+\infty}{\stackrel{\text { rapid }}{\longrightarrow}} m_{w_{I}} M_{\mathcal{L}}
$$

Thus, as $M_{\mathcal{L}}$ is compact and hence $M_{\mathcal{L}} / M_{\mathcal{L}, 0}$ is finite, one deduces from Lemma B.6 that:

$$
m_{s} M_{\mathcal{L}, 0} \underset{s \rightarrow+\infty}{\stackrel{\text { rapid }}{\longrightarrow}} m_{w_{I}} M_{\mathcal{L}, 0}
$$


But $M_{\mathcal{L}, 0} \subset M \cap H$. Hence

$$
m_{s}(M \cap H)_{0} \underset{s \rightarrow+\infty}{\stackrel{\mathrm{rapid}}{\longrightarrow}} m_{w_{I}}(M \cap H)_{0} .
$$

It follows from this that, for $s$ large enough,

$$
m_{w_{I}}^{-1} m_{s}=\left(\exp Y_{s}\right) m_{s}^{\prime},
$$

where $Y_{s}$ is an element of a supplementary subspace $\mathfrak{r}$ of $\mathfrak{m} \cap \mathfrak{h}$ in $\mathfrak{m}$ such that $\left(Y_{s}\right)$ converges rapidly to 0 , and $m_{s}^{\prime} \in(M \cap H)_{0}$. Note that $Y_{s}$ is unique and that $\left(m_{s}^{\prime}\right)$ converges to 1 .

Let us show that one can change $\left(m_{s}\right)$ into $\left(m_{s} m_{s}^{\prime-1}\right)$ in (4.16). One has $(M \cap H)_{0}=$ $\left(M \cap H_{w}\right)_{0}$ as the Lie algebra of these groups are the same. In fact $\mathfrak{l} \cap \mathfrak{h}_{w}=\mathfrak{l} \cap \mathfrak{h}$ (cf. [11, Lemma 3.7]) which implies easily that $\mathfrak{m} \cap \mathfrak{h}_{w}=\mathfrak{m} \cap \mathfrak{h}$. Hence $m_{s}^{\prime} \in M \cap H_{w}$. Then:

$$
\begin{array}{ll} 
& m_{s} w h_{s}=m_{s} m_{s}^{\prime-1} w w^{-1} m_{s}^{\prime} w h_{s} \\
\text { and } \quad & h_{s}^{\prime}=w^{-1} m_{s}^{\prime} w \in H, \text { as } m_{s}^{\prime} \in H_{w} .
\end{array}
$$

Hence, from (4.16), one gets:

$$
w_{I} \tilde{a}_{s}=u_{s} m_{s} m_{s}^{\prime-1} w h_{s}^{\prime} h_{s} .
$$

This proves our claim. One then deduces that one can choose $\left(m_{s}\right)$ such that $\left(m_{s}\right)$ converges rapidly to $m_{w_{I}}$. This achieves the proof of Proposition 5.1.

\subsection{End of proof of Proposition 4.11}

Let us continue the preparation of the proof of Proposition 4.11

5.13 Lemma. Let $\left(g_{s}^{\prime}\right)$ be a family in $G$ which converges rapidly to $g \in G$. Let $f \in$ $\mathcal{A}_{\text {temp,N }}(Z)$. Then there exist $C>0$ and $\varepsilon>0$ such that:

$$
\left|\left(L_{\left(g_{s}^{\prime}\right)^{-1}} f\right)(a)-\left(L_{g^{-1}} f\right)(a)\right| \leqslant C a_{s}^{\rho Q} e^{-\varepsilon s}, \quad a \in A_{Z}^{-}, s \geqslant s_{0} .
$$

Proof. As $\left(g_{s}^{\prime}\right)$ converges rapidly to $g$ when $s$ tends to $+\infty$, there exists $s_{0}^{\prime}, C^{\prime}, \varepsilon^{\prime}$ strictly positive and $\left(X_{s}\right) \subset \mathfrak{g}$ such that, for all $s \geqslant s_{0}^{\prime}$,

$$
g_{s}^{\prime}=g \exp X_{s} \text { and }\left\|X_{s}\right\| \leqslant C^{\prime} e^{-\varepsilon^{\prime} s} .
$$

As $L_{g^{-1}}$ preserves $\mathcal{A}_{\text {temp }, N}(Z)$, one is reduced to prove that, for all $f \in \mathcal{A}_{\text {temp }, N}(Z)$, there exist $C, \varepsilon, s_{0}>0$ such that:

$$
\left|f\left(\exp \left(X_{s}\right) a_{s}\right)-f\left(a_{s}\right)\right| \leqslant C a_{s}^{\rho_{Q}} e^{-\varepsilon s} .
$$

But, by the mean value Theorem, if $a \in A$ and $X \in \mathfrak{g}$,

$$
|f(\exp (X) a)-f(a)| \leqslant \sup _{t \in[0,1]}\left(L_{X} f(\exp (t X) a)\right)\|X\| .
$$


From (5.14), one then sees that it is enough to prove that, if $\|X\|$ is bounded by a constant $C^{\prime \prime}>0$, there exists a constant $C^{\prime \prime \prime}>0$ such that:

$$
\sup _{t \in[0,1]}\left\|L_{X} f(\exp (t X) a)\right\| \leqslant C^{\prime \prime \prime} a^{\rho_{Q}}(1+\|\log a\|)^{N}, \quad a \in A_{Z}^{-} .
$$

Decomposing $X$ in a basis $\left(X_{i}\right)$ of $\mathfrak{g}$ and using the continuity of the endomorphisms $L_{X_{i}}$ of $\mathcal{A}_{\text {temp }, N}(Z)$, one sees that there exists a continuous semi-norm such that:

$$
\left|\left(L_{X} f\right)(a)\right| \leqslant q(f) a^{\rho_{Q}}(1+\|\log a\|)^{N}, \quad a \in A_{Z}^{-}
$$

But $f \mapsto \sup _{\|X\| \leqslant C^{\prime \prime}} q\left(L_{\exp (-t X)} f\right)$ is a continuous semi-norm on $\mathcal{A}_{t e m p, N}(Z)$. Hence, as $L_{X}$ and $L_{\exp (-t X)}$ commute, (5.15) follows. This achieves to prove the Lemma.

Proof of Proposition 4.11. If $a \in A$, one has:

$$
\begin{aligned}
& \left(\left(L_{a} f\right)^{w}\right)_{I}=\left(L_{a}\left(f^{w}\right)\right)_{I} \text { as }\left(L_{a} f\right)^{w}=L_{a} f^{w} \\
& \text { and } \quad\left(L_{m_{w_{I}}}\left(L_{a} f\right)_{I}\right)^{w_{I}}=\left(L_{a} L_{m_{w_{I}}} f_{I}\right)^{w_{I}}=L_{a}\left(L_{m_{w_{I}}} f_{I}\right)^{w_{I}} .
\end{aligned}
$$

Hence it is enough to prove the identity of the Proposition for $a_{Z}=z_{0}$. Using (4.7) and Proposition 4.8, it is enough to prove that, for some family $\left(a_{s}\right)$ as in (4.13), that $s \mapsto$ $\left(L_{m_{w_{I}}} f_{I}\right)^{w_{I}}\left(a_{s}\right)$ is an exponential polynomial with unitary characters satisfying:

$$
\lim _{s \rightarrow+\infty} a_{s}^{-\rho_{Q}}\left(f^{w}\left(a_{s}\right)-\left(L_{m_{w_{I}}} f_{I}\right)^{w_{I}}\left(a_{s}\right)\right)=0
$$

But from (4.16),

$$
\tilde{a}_{s} w \cdot z_{0}=\left(\tilde{a}_{s} \tilde{b}_{s}^{-1} m_{s}^{-1} u_{s}^{-1}\right)\left(u_{s} m_{s} \tilde{b}_{s} w\right) \cdot z_{0}=g_{s} w_{I} \tilde{a}_{s} \cdot z_{0}
$$

for $s \geqslant s_{0}$, where $g_{s}=\tilde{a}_{s} \tilde{b}_{s}^{-1} m_{s}^{-1} u_{s}^{-1}$. Then one has:

$$
f^{w}\left(a_{s}\right)=L_{w_{I}^{-1} g_{s}^{-1}} f\left(a_{s}\right)
$$

On the other hand, from [11, Lemma 3.4] for $Z=Z_{I}$, as $A_{Z_{I}, E}=A_{I}$ (cf. loc.cit. equation (3.10)), one has:

$$
\tilde{a}_{s} w_{I} \cdot z_{0, I}=w_{I} \tilde{a}_{s} \cdot z_{0, I}
$$

which implies that:

$$
\left(L_{w_{I}^{-1} m_{w_{I}}} f_{I}\right)\left(\tilde{a}_{s} \cdot z_{0, I}\right)=\left(L_{m_{w_{I}}} f_{I}\right)^{w_{I}}\left(a_{s}\right) .
$$

Now, as $\left(g_{s} w_{I}\right)$ converges rapidly to $m_{w_{I}}^{-1} w_{I}$, we can apply Lemma 5.13 with $g_{s}^{\prime}=g_{s} w_{I}$ and find $C^{\prime}, \varepsilon^{\prime}, s_{0}^{\prime}>0$ such that:

$$
a_{s}^{-\rho_{Q}}\left|\left(L_{w_{I}^{-1} g_{s}^{-1}} f\right)\left(a_{s}\right)-\left(L_{w_{I}^{-1} m_{w_{I}}} f\right)\left(a_{s}\right)\right| \leqslant C^{\prime} e^{-\varepsilon^{\prime} s}, \quad s \geqslant s_{0}^{\prime} .
$$


Using Lemmas 4.5 and 4.9, one has, for some $C^{\prime \prime}, \varepsilon^{\prime}>0$,

$$
\left|a_{s}^{-\rho_{Q}}\left(L_{w_{I}^{-1} m_{w_{I}}} f\left(a_{s}\right)-L_{w_{I}^{-1} m_{w_{I}}} f_{I}\left(a_{s}\right)\right)\right| \leqslant C^{\prime \prime} e^{-\varepsilon^{\prime} s}, \quad s \geqslant s_{0}^{\prime} .
$$

Hence from (5.18) and (5.19), one deduces (5.16). It remains to prove that:

$$
s \mapsto\left(L_{m_{w_{I}}}\right) f_{I}^{w_{I}}\left(\exp \left(s X_{I}\right)\right)=f_{I}\left(m_{w_{I}}^{-1} \exp \left(s X_{I}\right) w_{I} \cdot z_{0, I}\right)
$$

is an exponential polynomial with unitary characters. But, from [11, Lemma 3.4] applied to $Z_{I}$,

$$
\left(L_{m_{w_{I}}} f_{I}\right)^{w_{I}}\left(\exp \left(s X_{I}\right)\right)=f_{I}\left(m_{w_{I}}^{-1} w_{I} \exp \left(s X_{I}\right)\right) .
$$

Hence our claim follows from(5.17). This achieves the proof of the Proposition.

\section{Transitivity of the constant term}

Let us notice that if $Z$ is wave-front, then, for $J \subset S, Z_{J}$ is not necessarily wave-front. Let us see that it is possible to define the constant term $f_{I}$ for $I \subset J$ and $f \in \mathcal{A}_{\text {temp }}\left(Z_{J}\right)$.

In particular, the characterization of $f_{I}$ will be given by the analogue of Proposition 4.8, say Proposition 4.8, with $Z$ changed in $Z_{J}$ and $\mathfrak{a}_{I}^{--}$changed in $\mathfrak{a}_{I, J}^{--}=\left\{X \in \mathfrak{a}_{I}: \alpha(X)<\right.$ $0, \alpha \in J \backslash I\}$. One has also an analogue of Theorem 4.13 (say Theorem 4.13'). To see this, one gets the analogues of Lemmas 3.1 and 3.5 where $Z$ is changed in $Z_{J}, \mathfrak{a}_{I}^{--}$in $\mathfrak{a}_{I, J}^{--}$and $\beta_{I}$ is changed in $\beta_{I, J}$ with:

$$
\beta_{I, J}(X)=\max _{\alpha \in J \backslash I} \alpha(X), \quad X \in \mathfrak{a}_{I, J}^{--} .
$$

In the proof one changes $\alpha+\beta \notin\langle I\rangle$ by $\alpha+\beta \in\langle J\rangle, \alpha+\beta \notin\langle I\rangle$. The rest of the proof is then entirely similar to the proof of Proposition 4.8 and Theorem 4.13. Let us notice that here we use Proposition 5.1 for a non wave-front spherical space.

6.1 Proposition. Let $I \subset J$ be two subsets of $S$. Then, if $f \in \mathcal{A}_{\text {temp }}(Z)$,

$$
f_{I}=\left(f_{J}\right)_{I}
$$

Proof. By G-equivariance of the maps:

$$
\begin{aligned}
\mathcal{A}_{\text {temp }}(Z) & \rightarrow \mathcal{A}_{\text {temp }}\left(Z_{I}\right) \\
f & \mapsto f_{I}
\end{aligned} \text { and } \begin{aligned}
\mathcal{A}_{\text {temp }}\left(Z_{J}\right) & \rightarrow \mathcal{A}_{\text {temp }}\left(Z_{I}\right) \\
f & \mapsto f_{I}
\end{aligned}
$$

it is enough to show that, if $f \in \mathcal{A}_{\text {temp }}(Z), f_{I}\left(z_{0, I}\right)=\left(f_{J}\right)_{I}\left(z_{0, I}\right)$. Recall that $\mathfrak{a}_{Z_{J}}=\mathfrak{a}_{Z}$ and

$$
\mathfrak{a}_{I}^{--}=\left\{X \in \mathfrak{a}_{I}: \alpha(X)<0, \alpha \in S \backslash I\right\}, \quad \mathfrak{a}_{I, J}^{--}=\left\{X \in \mathfrak{a}_{I}: \alpha(X)<0, \alpha \in J \backslash I\right\} .
$$

As $\mathfrak{a}_{I}=\left\{X \in \mathfrak{a}_{Z}: \alpha(X)=0, \alpha \in I\right\}$ and $\mathfrak{a}_{J}=\left\{X \in \mathfrak{a}_{Z}: \alpha(X)=0, \alpha \in J\right\}$, one has:

$$
\mathfrak{a}_{J} \subset \mathfrak{a}_{I}, \quad \mathfrak{a}_{I}^{--} \subset \mathfrak{a}_{Z}^{-}, \quad \mathfrak{a}_{I, J}^{--} \subset \mathfrak{a}_{Z}^{-}
$$


One remaks that $\mathfrak{a}_{I}^{--} \subset \mathfrak{a}_{I, J}^{--}$. Let $X \in \mathfrak{a}_{J}^{--}$and $Y \in \mathfrak{a}_{I}^{--}$. Then $X+Y \in \mathfrak{a}_{I}^{--}$.

Using Theorem 4.13(ii) applied successively to $(Z, I, f, X+Y, 1)$ and $(Z, J, f, X, \exp (T Y))$ instead of $\left(Z, I, f, X, a_{Z}\right)$, and finally the analogue Theorem 4.13'(ii) of Theorem 4.13(ii) for $\left(Z_{J}, I, f_{J}, Y, \exp (T X)\right)$, one gets that there exist $C>0$ and $\varepsilon>0$ such that, for all $T \geqslant 0$,

$$
\begin{aligned}
& \alpha_{T}\left\|f(\exp (T(X+Y)))-f_{I}(\exp (T(X+Y)))\right\| \leqslant C e^{-\varepsilon T} \\
& \alpha_{T}\left\|f(\exp (T Y) \exp (T X))-f_{J}(\exp (T Y) \exp (T X))\right\| \leqslant C e^{-\varepsilon T} \\
& \alpha_{T}\left\|f_{J}(\exp (T X) \exp (T Y))-\left(f_{J}\right)_{I}(\exp (T X) \exp (T Y))\right\| \leqslant C e^{-\varepsilon T},
\end{aligned}
$$

where $\alpha_{T}=e^{-T \rho_{Q}(X+Y)}$. Hence one concludes from the three inequalities above that:

$$
\alpha_{T}\left\|f_{I}(\exp (T(X+Y)))-\left(f_{J}\right)_{I}(\exp (T(X+Y)))\right\| \leqslant 3 C e^{-\varepsilon T}, \quad T \geqslant 0 .
$$

Hence $\alpha_{T} f_{I}(\exp (T(X+Y)))-\alpha_{T}\left(f_{J}\right)_{I}(\exp (T(X+Y)))$ tends to zero when $T$ goes to $+\infty$. But each term of this difference is an exponential polynomial in $T$ with unitary characters. Hence, according to (4.7), the difference of the two occurring exponential polynomials is identically zero. It implies, taking $T=0$, that $f_{I}\left(z_{0, I}\right)=\left(f_{J}\right)_{I}\left(z_{0, I}\right)$.

\section{Uniform estimates}

Let $L^{\prime}$ be a Levi subgroup of $G$ which contains $A$. Let $A_{L^{\prime}}$ be a maximal vector subgroup of the center of $L^{\prime}$ contained in $A$. Recall that $\mathfrak{t}$ is a maximal abelian subalgebra of $\mathfrak{m}$. Let $\mathfrak{j}=i \mathfrak{t} \oplus \mathfrak{a}$ so that $\mathfrak{j}_{\mathbb{C}}$ is a complex Cartan subalgebra of $\mathfrak{g}_{\mathbb{C}}$. Let us notice that the Weyl group $W\left(\mathfrak{g}_{\mathbb{C}}, \mathfrak{t}_{\mathbb{C}}\right)$ preserves $\mathfrak{j}$. One has

$$
\mathfrak{j}=V \oplus U=V_{1} \oplus U_{1},
$$

where $V=\mathfrak{a}_{L_{I}}, V_{1}=\mathfrak{a}_{L^{\prime}}, U=i \mathfrak{t} \oplus\left(\mathfrak{a} \cap{ }^{0} \mathfrak{l}_{I}\right)$ and $U_{1}=i \mathfrak{t} \oplus\left(\mathfrak{a} \cap{ }^{0} \mathfrak{l}^{\prime}\right)$ (cf. (3.3) for the definition of $\left.{ }^{0} \mathfrak{l}_{I},{ }^{0} \mathfrak{l}^{\prime}\right)$.

In the following, we will apply Lemma A.1 to the map:

$$
\begin{aligned}
\mathfrak{j}_{\mathbb{C}}^{*} \times \mathfrak{a}_{L_{I}} & \rightarrow \operatorname{End}(W) \\
(\omega, X) & \mapsto-{ }^{t} \rho_{\omega}(X),
\end{aligned}
$$

where the notation has been defined after (3.10) and in (3.24).

From (3.12), one sees that the eigenvalues of $\Gamma_{\omega}(X)$ are of the form $-w \omega(X)$ for $w \in$ $W\left(\mathfrak{g}_{\mathbb{C}}, \mathfrak{j}_{\mathbb{C}}\right)$.

Let $\Lambda \in \mathfrak{t}_{\mathbb{C}}^{*} \oplus\left(\mathfrak{a} \cap{ }^{0} \mathfrak{l}^{\prime}\right)_{\mathbb{C}}^{*}$ fixed. Let $\nu \in i \mathfrak{a}_{L^{\prime}}^{*}$ and $\lambda \in \mathfrak{a}_{I}^{*}$. We set $\Lambda_{\nu}=\Lambda+\nu \in \mathfrak{j}_{\mathbb{C}}^{*}$. Let us look to the sum of the joint spectral projections of the $\Gamma_{\Lambda_{\nu}}(X), X \in \mathfrak{a}_{I}$, for a joint eigenvalue with real part equal to $\lambda$.

Let $\mathcal{I}_{\Lambda_{\nu}}$ be the kernel of the character $\chi_{\Lambda_{\nu}}$ of $Z(\mathfrak{g})$ given by the composition of the Harish-Chandra isomorphism from $Z(\mathfrak{g})$ onto $S\left(\mathfrak{j}_{\mathbb{C}}\right)^{W\left(\mathfrak{g}_{\mathbb{C}}, \mathfrak{j}_{\mathbb{C}}\right)}$ with the evaluation at $\Lambda_{\nu}$. Let us recall the notation introduced at Sections 3.1 and 3.5. Write $\mathcal{A}_{\text {temp }}\left(Z: \Lambda_{\nu}\right)$ instead of $\mathcal{A}_{\text {temp }}\left(Z: \mathcal{I}_{\Lambda_{\nu}}\right)$. 
We want to make Lemma 3.3(ii) more precise in this case. To do so, it is better to group spectral projections. We denote this sum by $E_{\lambda, \nu}$.

Let $\mathcal{Q}_{\Lambda}=\left\{-\operatorname{Re} w \Lambda \circ s_{\mid \tilde{\mathfrak{a}}_{I}}: w \in W\left(\mathfrak{g}_{\mathbb{C}}, \mathfrak{j}_{\mathbb{C}}\right)\right\}$. Identifying $\mathfrak{a}_{I}^{*}$ and $\tilde{\mathfrak{a}}_{I}^{*}$ by $s$, from (3.12), one sees that:

$$
E_{\lambda, \nu} \neq 0 \text { implies } \lambda \in \mathcal{Q}_{\Lambda}
$$

7.1 Lemma. Let $\lambda \in \mathcal{Q}_{\Lambda}$.

(i) There exists $\varepsilon>0$ such that $\nu \mapsto E_{\lambda, \nu}$ extends to a holomorphic function on

$$
\mathfrak{a}_{L^{\prime}, \varepsilon}^{*}=\left\{\nu \in \mathfrak{a}_{L^{\prime}, \mathbb{C}}^{*}:\|\operatorname{Re} \nu\|<\varepsilon\right\} .
$$

(ii) There exists $C>0$ and $q \in \mathbb{N}$ such that:

$$
\left\|E_{\lambda, \nu}\right\| \leqslant C(1+\|\nu\|)^{q}, \quad \nu \in i \mathfrak{a}_{L^{\prime}}^{*}
$$

(iii) There exists $C>0$ and $r \in \mathbb{N}$ such that:

$$
\left\|E_{\lambda, \nu} e^{\Gamma_{\Lambda_{\nu}}(X)}\right\| \leqslant C e^{\lambda(X)}(1+\|\nu\|)^{r}(1+\|X\|)^{r}, \quad \nu \in i \mathfrak{a}_{L^{\prime}}^{*}, X \in \mathfrak{a}_{I} .
$$

Proof. As $\rho_{\Lambda_{\nu}}$ is a representation of the abelian Lie algebra $\mathfrak{a}_{L_{I}}$, hence of $\tilde{\mathfrak{a}}_{I}$, the spectral projection $E_{\lambda, \nu}$ is equal (following the notation of Appendix A) to the product:

$$
\prod_{j=1}^{k} P_{\lambda\left(X_{j}\right), X_{j}}(-i \nu),
$$

where $X_{1}, \ldots, X_{k}$ is a basis of $\mathfrak{a}_{I}$. Then the assertions (i) and (ii) follow immediately from Lemma A.1.

Let us show (iii). One remarks that:

$$
E_{\lambda, \nu} e^{\Gamma_{\Lambda_{\nu}}(X)}=e^{E_{\lambda, \nu} \Gamma_{\nu}(X)} .
$$

The norm of $E_{\lambda, \nu}$ has a bound given by (i) and the norm of $\Gamma_{\Lambda_{\nu}}(X)$ is bounded by a constant times $(1+\|\nu\|)^{l}(1+\|X\|)$, as $\Gamma_{\Lambda_{\nu}}(X)$ is polynomial in $\nu$ and linear in $X$. Then (iii) follows from [16, Lemma 12.A.2.4].

For $\lambda \in \mathcal{Q}_{\Lambda_{\nu}}$, set

$$
E_{\lambda, \nu}(X)=e^{-\operatorname{Re} \lambda(X)} E_{\lambda, \nu}\left(e^{\Gamma_{\Lambda}(X)}\right), \quad X \in \mathfrak{a}_{I} .
$$

One has the analogue of Lemma 3.3(ii).

7.2 Lemma. Let $N \in \mathbb{N}$. If, for any $\nu \in i \mathfrak{a}_{L^{\prime}}^{*}, \lambda \in \mathcal{Q}_{\Lambda_{\nu}}$ and $f \in \mathcal{A}_{\text {temp }, N}\left(Z: \Lambda_{\nu}\right)$, one sets:

$$
\Phi_{f, \lambda, \nu}=E_{\lambda, \nu} \Phi_{f}
$$

then one has:

$$
\begin{aligned}
\Phi_{f, \lambda, \nu}\left(a_{Z} \exp \left(T X_{I}\right)\right)= & e^{T \Gamma_{\Lambda_{\nu}}\left(X_{I}\right)} \Phi_{f, \lambda, \nu}\left(a_{Z}\right) \\
& +\int_{0}^{T} E_{\lambda, \nu} e^{(T-t) \Gamma_{\Lambda_{\nu}}\left(X_{I}\right)} \Psi_{f, X_{I}}\left(a_{Z} \exp \left(t X_{I}\right)\right) d t \\
& a_{Z} \in A_{Z_{I}}, X_{I} \in \mathfrak{a}_{I} .
\end{aligned}
$$


7.3 Lemma. Let $X \in \mathfrak{a}_{I}$. There exist a continuous semi-norm $q$ on $C_{\text {temp,N }}^{\infty}(Z)$ and $m \in \mathbb{N}$ such that, for all $\nu \in i \mathfrak{a}_{L^{\prime}}^{*}$ and $f \in \mathcal{A}_{\text {temp }, N}\left(Z: \Lambda_{\nu}\right)$,

$$
\left\|\Psi_{f, X}\left(a_{Z} \exp X_{I}\right)\right\| \leqslant q(f)\left(1+\left\|\log a_{Z}\right\|\right)^{N}\left(1+\left\|X_{I}\right\|\right)^{N}(1+\|\nu\|)^{m}, \quad a_{Z} \in A_{Z}^{-}, X_{I} \in \mathfrak{a}_{I}^{--} .
$$

Proof. The proof is the same than the proof of Lemma 3.5(ii), the factor $(1+\|\nu\|)^{m}$ coming from (3.11).

One has an analogue of Lemma 3.6, where $f \in \mathcal{A}_{\text {temp,N }}\left(Z: \Lambda_{\nu}\right), \lambda \in \mathcal{Q}_{\Lambda}$ and $E_{\lambda}$ is replaced by $E_{\lambda, \nu}$. The proof is the same using Lemma 7.1(iii) instead of (3.26).

One introduces $\Phi_{f, \lambda, \nu, \infty}$ as in (3.27) by replacing $\Phi_{f, \lambda}$ by $\Phi_{f, \lambda, \nu}$ (and $\lambda \in \mathcal{Q}_{\Lambda}$ instead of $\mathcal{Q}_{\mathcal{I}}$ ). Similarly one has an analogue of Lemma 3.8.

We also define a partition of $\mathcal{Q}_{\Lambda}$ into three disjoint sets $\mathcal{Q}_{\Lambda}^{-}, \mathcal{Q}_{\Lambda}^{0}$ and $\mathcal{Q}_{\Lambda}^{+}$. Then one has analogue of Lemma 3.9, Corollary 3.10, Lemmas 3.11 and 3.12, and Proposition 3.14, which are valid for all $\nu \in i \mathfrak{a}_{I}^{*}$ and all $f \in \mathcal{A}_{t e m p, N}\left(Z: \Lambda_{\nu}\right)$, by replacing $\Phi_{\lambda}$ by $\Phi_{f, \lambda, \nu}, E_{\Lambda}$ by $E_{\lambda, \nu}$ and $\Phi_{\lambda, \infty}$ by $\Phi_{f, \lambda, \nu, \infty}$.

7.4 Theorem. Let $L^{\prime}$ be a Levi subgroup of $G$ containing $A, \mathcal{C}$ be a compact subset of $\mathfrak{a}_{I}^{--}$ and $\Omega_{1}$ be a compact subset of $G$. Let $N \in \mathbb{N}$.

(i) There exist $\varepsilon>0, m \in \mathbb{N}$ and a continuous semi-norm $p$ on $C_{\text {temp,N }}^{\infty}(Z)$ such that, for all $\nu \in i \mathfrak{a}_{L^{\prime}}^{*}$ and all $f \in \mathcal{A}_{\text {temp }, N}\left(Z: \Lambda_{\nu}\right)$, one has:

$$
\begin{aligned}
& \left(a_{Z} \exp (T X)\right)^{-\rho_{Q}}\left|f\left(\omega a_{Z} \exp (T X)\right)-f_{I}\left(\omega a_{Z} \exp (T X)\right)\right| \\
& \leqslant e^{-\varepsilon T} p(f)\left(1+\left\|\log a_{Z}\right\|\right)^{N}(1+\|\nu\|)^{m}, \quad a_{Z} \in A_{Z}^{-}, X \in \mathcal{C}, \omega \in \Omega_{1}, T \geqslant 0 .
\end{aligned}
$$

(ii) Let $q$ be a continuous semi-norm on $C_{\text {temp }, N+\operatorname{dim}(W)}^{\infty}\left(Z_{I}\right)$. Then there exists a continuous semi-norm p on $C_{\text {temp }, N}^{\infty}(Z)$ such that

$$
q\left(f_{I}\right) \leqslant p(f)(1+\|\nu\|)^{m}, \quad \nu \in i \mathfrak{a}_{L^{\prime}}^{*}, f \in \mathcal{A}_{\text {temp }, N}\left(Z: \Lambda_{\nu}\right) .
$$

Proof. To get (i), one needs an analogue of Lemma 4.1. Due to the occurrence of powers of $(1+\|\nu\|)$ in Lemmas 7.3, 7.1(ii) and (iii), one gets:

7.5 Lemma. We fix a compact set $\mathcal{C}$ in $\mathfrak{a}_{I}^{--}$and choose $\varepsilon_{0}>0$ such that $\beta_{I}(X) \leqslant-2 \varepsilon_{0}$ for $X \in \mathfrak{a}_{I}^{--}$. We put $\varepsilon=\delta \varepsilon_{0}$, with $\delta$ given by (3.29). Then there exist $m \in \mathbb{N}$ and a continuous semi-norm $p$ on $C_{\text {temp }, N}^{\infty}(Z)$ such that, for all $\nu \in i \mathfrak{a}_{L^{\prime}}^{*}$ and all $f \in \mathcal{A}_{\text {temp }, N}\left(Z: \Lambda_{\nu}\right)$, one has:

$$
\begin{array}{r}
\left\|\Phi_{f, \lambda, \nu}\left(a_{Z} \exp (T X)\right)-\Phi_{f, \lambda, \infty}\left(a_{Z} \exp (T X)\right)\right\| \leqslant e^{-\varepsilon T} p(f)\left(1+\left\|\log a_{Z}\right\|\right)^{N}(1+\|\nu\|)^{m} \\
a_{Z} \in A_{Z}^{-}, X \in \mathcal{C}, T \geqslant 0
\end{array}
$$

Then, using Lemma 7.5 instead of Lemma 4.1, the proof of Theorem 7.4(i) is similar to the proof of Theorem 4.13(ii). 
The proof of (ii) is analogous to the proof of Theorem 4.13(i), keeping track on the dependence on $\nu$. The cornerstone is Lemma 4.10 relying on Lemma 4.3. Looking to the proof of the later, it is based on Lemmas 3.5(i) and 4.1. But the dependence of Lemma 4.1 with the parameter $\nu$ is given by (i) above. Moreover, as $W=W_{\mathcal{I}}$ for $\mathcal{I}=\mathcal{I}_{\Lambda_{\nu}}$, in Lemma 3.5(i), the proof shows that the semi-norm $p_{u}$ does not depend on $\mathcal{I}=\mathcal{I}_{\Lambda_{\nu}}$ if $\nu \in i \mathfrak{a}_{L^{\prime}}^{*}$. This leads to our claim.

\section{A Variation of a Lemma due to N. Wallach}

We will need a mild variation of Lemma 12.A.2.9 in [16].

Let $E$ be a finite dimensional vector space over $\mathbb{R}$ and assume that $U, V, U_{1}$ and $V_{1}$ are real vector subspaces such that $E=V \oplus U=V_{1} \oplus U_{1}$. If $\nu \in U_{\mathbb{C}}^{*}$ or $\nu \in U_{1, \mathbb{C}}^{*}$ (resp. $V_{\mathbb{C}}^{*}$ or $\left.V_{1, \mathbb{C}}^{*}\right)$ we extend $\nu$ to $E$ by $\nu(V)=0$ or $\nu\left(V_{1}\right)=0\left(\right.$ resp. $\nu(U)=0$ or $\left.\nu\left(U_{1}\right)=0\right)$. If $\Lambda \in E_{\mathbb{C}}^{*}$ and $\Lambda=\Lambda_{1}+i \Lambda_{2}$ with $\Lambda_{1}, \Lambda_{2} \in E^{*}$, we set $\Lambda_{1}=\operatorname{Re} \Lambda, \Lambda_{2}=\operatorname{Im} \Lambda$.

Let $B: E_{\mathbb{C}}^{*} \times V \rightarrow M_{n}(\mathbb{C})$ be a map which is polynomial in the first variable and linear in the second. We assume that there exist $s_{1}, \ldots, s_{r} \in \mathrm{GL}\left(E_{\mathbb{C}}^{*}\right)$ such that the eigenvalues of $B(\Lambda, v), v \in V$, are of the form $s_{j} \Lambda(v)$.

We fix $\Lambda_{1} \in U_{1, \mathbb{C}}^{*}$ (and not in $U^{*}$ as in loc.cit.). We fix linear coordinates $\left\{x_{1}, \ldots, x_{n}\right\}$ on $V$ and we will use the multi-index notation for partial derivatives.

If $\mu \in \mathbb{R}, \nu \in V_{1}^{*}$ and $v \in V$, let $P_{\mu, v}(\nu)$ be the projection onto the sum of generalized eigenspaces for $B\left(\Lambda_{1}+i \nu, v\right)$ with eigenvalues having real part equal to $\mu$.

A.1 Lemma. Let $v \in V$ and $\mu \in \mathbb{R}$.

(i) The map $\nu \mapsto P_{\mu, v}(\nu)$ is real analytic on $V_{1}^{*}$. Even more, there exists $\varepsilon_{1}>0$ such that $P_{\mu, v}$ extends to an holomorphic function on $V_{1, \varepsilon_{1}}^{*}=\left\{\nu \in V_{1, \mathbb{C}}^{*}:\|\operatorname{Im} \nu\|<\varepsilon_{1}\right\}$.

(ii) There exists $q \in \mathbb{N}$ such that, for any $I \in \mathbb{N}^{m}$, there exists $c_{I}>0$ such that:

$$
\left\|\partial^{I} P_{\mu, v}(\nu)\right\| \leqslant c_{I}(1+\|\nu\|)^{p}, \quad \nu \in V_{1}^{*} .
$$

Proof. We give a complete proof in order to take care of the change and repair small misprints in the proof of [16, Lemma 12.A.2.9].

If $\operatorname{Re} s_{j} \Lambda_{1}(v) \neq \mu$ for all $j$, then $P_{\mu, v} \equiv 0$ and there is nothing to prove.

Otherwise, after we reorder the $s_{j}$ 's, we may assume that there exists some $0<m \leqslant r$ such that:

$$
\operatorname{Re} s_{1} \Lambda_{1}(v)=\cdots=\operatorname{Re} s_{m} \Lambda_{1}(v)=\mu \text { and } \operatorname{Re} s_{j} \Lambda_{1}(v) \neq \mu \text { for } j>m \text {. }
$$

Let $0<\varepsilon \leqslant 1 / 2 \min _{j>m}\left|\operatorname{Re} s_{j} \Lambda_{1}(v)-\mu\right|$ and let $\nu_{0} \in V_{1}^{*}$ be fixed. There exists $R\left(\nu_{0}\right)>0$ such that the interior $O$ of the rectangle $\bar{O}$ in $\mathbb{C}$ of center $\mu$, width $2 \varepsilon$ and height $2 R\left(\nu_{0}\right)$ is such that $\nu_{0}$ satisfies the property $\left(\mathcal{P}_{\nu}\right)$, for $\nu=\nu_{0}$, given by:

$$
s_{j}\left(\Lambda_{1}+i \nu\right)(v) \in \bar{O} \text { if and only if } j \leqslant m \text { and in that case } s_{j}(\Lambda+i \nu)(v) \in O \text {. }
$$


One can take $R\left(\nu_{0}\right)=\max _{j \leqslant r}\left|s_{j}\left(\operatorname{Im}\left(\Lambda_{1}\right)+\nu_{0}\right)(v)\right|+1$. The reason to add 1 is to ensure $R\left(\nu_{0}\right)>0$. Let us notice that there is a constant $c_{1}>0$ such that:

$$
R\left(\nu_{0}\right) \leqslant c_{1}\left(1+\left\|\nu_{0}\right\|\right)
$$

Let $\Omega\left(\nu_{0}\right)=\left\{\nu \in V_{1}^{*}: \max _{j \leqslant r}\left|s_{j}\left(\operatorname{Im}\left(\Lambda_{1}\right)+\nu\right)(v)\right|<R\left(\nu_{0}\right)\right\}$. Then $\Omega\left(\nu_{0}\right)$ is open and contains $\nu_{0}$.

Let $\omega_{\varepsilon}:=\left\{\nu \in V_{1}^{*}:\left|s_{j}(\nu)(v)\right|<\varepsilon / 2, j \leqslant r\right\}$ and let $\Omega_{\varepsilon}\left(\nu_{0}\right):=\left\{\nu_{1}+i \nu_{2}: \nu_{1} \in \omega_{\varepsilon}, \nu_{2} \in\right.$ $\left.\Omega\left(\nu_{0}\right)\right\}$. Then, if $\nu \in \Omega_{\varepsilon}\left(\nu_{0}\right)$,

$$
\begin{array}{ll}
\left|\operatorname{Re} s_{j}\left(\Lambda_{1}+\nu\right)(v)-\mu\right|<\varepsilon, & j=1, \ldots, m, \\
\left|\operatorname{Re} s_{j}\left(\Lambda_{1}+\nu\right)(v)-\mu\right|>\varepsilon, & j=m+1, \ldots, r .
\end{array}
$$

This implies that, if $\nu \in \Omega_{\varepsilon}\left(\nu_{0}\right)$, then $\left(\mathcal{P}_{\nu}\right)$ is satisfied.

Let $C$ be the boundary of $O$ and $\nu \in \Omega_{\varepsilon}\left(\nu_{0}\right)$. Let

$$
Q(\nu)=\frac{1}{2 i \pi} \int_{C}\left(B\left(\Lambda_{1}+i \nu, v\right)-z I d\right)^{-1} d z, \quad \nu \in \Omega_{\varepsilon}\left(\nu_{0}\right) .
$$

Then, if $\nu \in \Omega\left(\nu_{0}\right), P_{\mu, v}(\nu)=Q(\nu)$. Moreover $\nu \mapsto Q(\nu)$ is holomorphic on $\Omega_{\varepsilon}\left(\nu_{0}\right)$. Varying $\nu_{0}$, it implies that $Q(\nu)$ is holomorphic on $\Omega_{\varepsilon}\left(\nu_{0}\right)$ by $\Omega^{\prime}:=\left\{\nu_{1}+i \nu_{2} \in V_{1, \mathbb{C}}^{*}: \nu_{1} \in \omega_{\varepsilon}, \nu_{2} \in V_{1}^{*}\right\}$. But it is clear that $\Omega^{\prime}$ contains $V_{1, \varepsilon_{1}}^{*}$ for some $\varepsilon_{1}>0$, as $\omega_{\varepsilon}$ is a neighborhood of 0 in $V_{1}^{*}$. This proves (i).

Let us fix $\nu \in \Omega_{\varepsilon}\left(\nu_{0}\right)$. We can write $B\left(\Lambda_{1}+i \nu, v\right)=U(D+N) U^{-1}$ (cf. [16, Lemma 12.A.2.2]), where $D, N, U \in M_{n}(\mathbb{C})$ are such that $D$ is diagonal, $N$ is upper triangular with zeros on the main diagonal and $U$ is unitary.

As the eigenvalues of $D$ are of the form $s_{k}\left(\Lambda_{1}+i \nu\right)(v)$ and as we use the Hilbert-Schmidt norm on $M_{n}(\mathbb{C})$ as all norms on $M_{n}(\mathbb{C})$ are equivalent, hence $\left\|B\left(\Lambda_{1}+i \nu, v\right)\right\|=\|D+N\|$. For the purpose of our estimate, we may assume $B\left(\Lambda_{1}+i \nu, v\right)=D+N$.

Let $z \in C$. As one can write:

$$
(D+N-z I)=(D-z I)\left(I+(D-z I)^{-1} N\right)
$$

and $N$ is nilpotent, one can obtain:

$$
(D+N-z I)^{-1}=\left(\sum_{k=0}^{n-1}(-1)^{k}\left((D-z I)^{-1} N\right)^{k}\right)(D-z I)^{-1} .
$$

If $z \in C$, for all $k$,

$$
\left|s_{k}\left(\Lambda_{1}+i \nu\right)(v)-z\right| \geqslant \varepsilon / 2 .
$$

Then, as we use the Hilbert-Schmidt norm,

$$
\left\|(D-z I)^{-1}\right\| \leqslant 2 n^{1 / 2} / \varepsilon .
$$

From the equality $B\left(\Lambda_{1}+i \nu, v\right)=D+N$, one gets:

$$
\|N\| \leqslant\left\|B\left(\Lambda_{1}+i \nu, v\right)\right\|
$$


From the polynomial behavior of $B$ in the first variable, there exists $c_{2}>0$ and $q \in \mathbb{N}$ such that:

$$
\left\|B\left(\Lambda_{1}+i \nu, v\right)\right\| \leqslant c_{2}(1+\|\nu\|)^{q}, \quad \nu \in V_{2}^{*} .
$$

There exists $c_{3}>0$ such that, for all $x>0$,

$$
\left(1+\cdots+x^{n-1}\right) \leqslant c_{3}\left(1+x^{n-1}\right) .
$$

Hence

$$
\left\|(D+N-z I)^{-1}\right\| \leqslant c_{3} \frac{2 n^{1 / 2}}{\varepsilon}\left(1+c_{2}(1+\|\nu\|)^{q(n-1)}\right) .
$$

Now we want to have a bound of $\|Q(\nu)\|$. It remains to bound the length of the contour $C$. This is bounded by $4 R\left(\nu_{0}\right)+4 \varepsilon$ and $\left\|R\left(\nu_{0}\right)\right\| \leqslant c_{1}\left(1+\left\|\nu_{0}\right\|\right)(1+\|v\|)$. Hence one sees easily that there exists $c_{4}>0$ such that:

$$
\|Q(\nu)\| \leqslant c_{4}\left(1+\left\|\nu_{0}\right\|\right)(1+\|\nu\|)^{q(n-1)}, \quad \nu \in \Omega_{\varepsilon}\left(\nu_{0}\right)
$$

Applying this inequality for $\nu=\nu_{0}$, one gets:

$$
\left\|Q\left(\nu_{0}\right)\right\| \leqslant c_{4}\left(1+\left\|\nu_{0}\right\|\right)^{q(n-1)+1}
$$

To deal with arbitrary derivative $\partial^{I}$, one has to use the Cauchy integral formula.

\section{B Rapid convergence}

B.1 Definition. Let $a \geqslant 0$ and $\left(x_{s}\right)$ be a family of elements of a normed vector space with $s \in\left[a,+\infty\left[\right.\right.$. One says that $\left(x_{s}\right)$ converges rapidly to $l$ if

$$
\begin{gathered}
\text { there exist } \varepsilon>0, C>0, s_{0} \in\left[a,+\infty\left[\text { such that, for any } s \geqslant s_{0}\right.\right. \\
\left\|x_{s}-l\right\| \leqslant C e^{-\varepsilon s} .
\end{gathered}
$$

To shorten, we will write $x_{s} \underset{s \rightarrow \infty}{\stackrel{\text { rapid }}{\longrightarrow}}$ l.

B.2 Lemma. Let $a \geqslant 0, E$ and $F$ be two Euclidean spaces, $l \in E$. Let $\phi$ be an F-valued map which is of class $C^{1}$ on a neighborhood $U$ of $l$ and such that the differential $\phi^{\prime}(l)$ of $\Phi$

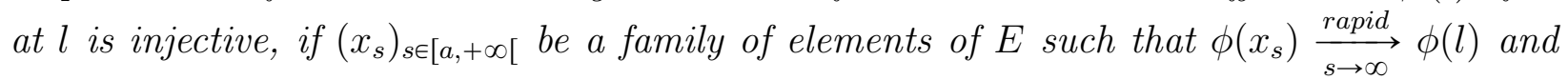
$\left(x_{s}\right)$ converges to $l$ when $s$ tends to $+\infty$. Then

$$
x_{s} \underset{s \rightarrow \infty}{\stackrel{\text { rapid }}{\longrightarrow}} l \text {. }
$$

Proof. Let $G$ be a supplementary of the image of $\phi^{\prime}(l)$ in $F$ and consider the map:

$$
\begin{aligned}
\Phi: E \times G & \rightarrow F \\
(x, z) & \mapsto \phi(x)+z .
\end{aligned}
$$


As $\phi^{\prime}(x)$ is injective, $\Phi^{\prime}(l, z)$ is injective and $\operatorname{dim}(E \times G)=\operatorname{dim}(F)$. Hence $\Phi^{\prime}(l, z)$ is invertible for any $z \in G$. From the local inversion theorem, $\Phi$ is then bijective on its image and of class $C^{1}$ on a neighborhood $V \times W$ of $(l, z)$ contained in $U \times G$. Consider the restriction $\widetilde{\Phi}$ of $\Phi$ to $V \times W$. Then $\widetilde{\Phi}$ is well-defined and of class $C^{1}$. Applying the Taylor expansion of $\widetilde{\Phi}^{-1}$ at $\Phi(l, 0)=\phi(l)$, one has for $s$ large enough such that $x_{s} \in V$ :

$$
\begin{aligned}
\left\|x_{s}-l\right\| & =\left\|\widetilde{\Phi}^{-1}\left(\phi\left(x_{s}\right)\right)-\widetilde{\Phi}^{-1}(\phi(l))\right\| \\
& \leqslant\left\|\left(\widetilde{\Phi}^{-1}\right)^{\prime}(\phi(l))\right\|\left\|\phi\left(x_{s}\right)-\phi(l)\right\|+o\left(\left\|\phi\left(x_{s}\right)-\phi(l)\right\|\right) .
\end{aligned}
$$

Our claim follows from the rapid convergence of $\left(\phi\left(x_{s}\right)\right)$.

B.3 Definition. Let $a \geqslant 0, X$ be a d-dimensional smooth manifold and $\left(x_{s}\right)_{[a,+\infty[}$ be a family of elements of $X$. One says that $\left(x_{s}\right)$ converges rapidly in $X$ if there exist $l \in X$ and a chart $(U, \phi)$ around $l$ such that

$$
\left(\phi\left(x_{s}\right)\right) \text { converges rapidly to } \phi(l) \text {. }
$$

B.4 Remark. This notion is independent of the choice of the chart $(U, \phi)$. Indeed, let $(\tilde{U}, \tilde{\phi})$ be another chart around $l$. Then, from Lemma B.2, $\left((\phi \circ \tilde{\phi})^{-1}\left(\phi\left(x_{s}\right)\right)\right)$ converges rapidly to $\tilde{\phi}(l)$ over $F$ which means that $\left(\tilde{\phi}\left(x_{s}\right)\right)$ converges rapidly to $\tilde{\phi}(l)$ over $F$. Also $\Psi: X \rightarrow Y$ is a differentiable map between $C^{\infty}$ manifolds and $\left(x_{s}\right)$ converges rapidly to $x$ in $X$, then $\Psi\left(\left(x_{s}\right)\right)$ converges rapidly to $\Psi(x)$ in $Y$.

B.5 Lemma. Let $X$ and $Y$ be two smooth manifolds, $l \in X$ and $\varphi$ be an $Y$-valued smooth map on a neighborhood $U$ of $l$ in $X$ such that $\varphi^{\prime}(l)$ is injective.

If $\left(x_{s}\right)_{s \in[a,+\infty}$ is a family of elements of $U$ converging to $l$ when $s$ tends to $+\infty$ and such that $\left(\varphi\left(x_{s}\right)\right)$ converges rapidly to $\varphi(l)$, then $\left(x_{s}\right)$ converges rapidly to $l$.

Proof. By taking charts in a neighborhood of $l$ and $\varphi(l)$, one is reduced to the case where $X$ and $Y$ are Euclidean spaces. Then the lemma follows from Lemma B.2 and Definition B.3.

B.6 Lemma. Let $a \geqslant 0$. Let $G$ be a Lie group and $R$ a closed subgroup of $G$ such that $R / R_{0}$ is finite. Let $\left(g_{s}\right)_{s \in[a,+\infty[}$ be a family in $G$ and $g \in G$ such that $g_{s} \underset{s \rightarrow+\infty}{\longrightarrow} g$ and

$$
g_{s} R \underset{s \rightarrow \infty}{\stackrel{\text { rapid }}{\longrightarrow}} g R .
$$

Then one has:

$$
g_{s} R_{0} \underset{s \rightarrow \infty}{\stackrel{\text { rapid }}{\longrightarrow}} g R_{0} .
$$

Proof. By multiplying on the left by $g^{-1}$, on can reduce to the case where $g=1$. Let $\mathfrak{q}$ be a supplementary of $\mathfrak{r}$ in $\mathfrak{g}$ and $b \geqslant a$ such that, for any $b \geqslant a, g_{s} R=e^{X_{s}} R$ where $\left(X_{s}\right)$ is a family in $\mathfrak{q}$ which converges rapidly to 0 . Hence, for any $s \geqslant b, g_{s}=e^{X_{s}} r_{s}$, where $\left(r_{s}\right)$ is a family in $R$. As $\left(g_{s}\right)$ converges to 1 , one has also that $\left(r_{s}\right)$ converges to 1 . Hence, as $R_{0}$ is open, $r_{s} \in R_{0}$ for $s$ large enough. This proves the Lemma. 
B.7 Lemma. Let $V$ be a finite dimensional vector space over $\mathbb{R}$ and $\mathbf{P}_{\mathbb{R}}(V)$ be its real projective space. Let $s_{0} \geqslant 0$ and let $\left(v_{s}\right)_{s \geqslant s_{0}},\left(w_{s}\right)_{s \geqslant s_{0}}$ be two families of vectors in $V$ such that, when $s \rightarrow+\infty$ :

(i) $\left(v_{s}\right)$ converges to a non zero element $v$;

(ii) $\left(v_{s}-w_{s}\right)$ converges rapidly to zero;

(iii) $\left(\left[w_{s}\right]\right)$ tends rapidly to $[w]$ in $\mathbf{P}_{\mathbb{R}}(V)$.

Then $\left[v_{s}\right] \underset{s \rightarrow+\infty}{\stackrel{\mathrm{rapid}}{\longrightarrow}}[w]$.

Proof. By dividing $v_{s}$ and $w_{s}$ by $\left\|v_{s}\right\|$, one can reduce to the case where $\left\|v_{s}\right\|=1$. Then $w_{s}=v_{s}+\varepsilon_{s}$ with $\left\|\varepsilon_{s}\right\| \leqslant C e^{-\varepsilon s}$ for some $C>0$ and $\varepsilon>0$. As $\left\|v_{s}\right\|-\left\|\varepsilon_{s}\right\| \leqslant\left\|w_{s}\right\| \leqslant\left\|v_{s}\right\|+\left\|\varepsilon_{s}\right\|$, $\left\|w_{s}\right\|=1+\varepsilon_{s}^{\prime}$ with $\left|\varepsilon_{s}^{\prime}\right| \leqslant\left\|\varepsilon_{s}\right\|$. Then $\frac{w_{s}}{\left\|w_{s}\right\|}-v_{s}=\frac{w_{s}-v_{s}\left(1+\varepsilon_{s}^{\prime}\right)}{1+\varepsilon_{s}^{\prime}}$ implies for $s$ large enough,

$$
\begin{aligned}
\left\|\frac{w_{s}}{\left\|w_{s}\right\|}-v_{s}\right\| & \leqslant \frac{\left\|\varepsilon_{s}\right\|+\left|\varepsilon_{s}^{\prime}\right|}{1+\varepsilon_{s}^{\prime}} \\
& \leqslant 2 C e^{-\varepsilon s}
\end{aligned}
$$

for $s$ large enough. Hence $\frac{w_{s}}{\left\|w_{s}\right\|^{\prime}}-v_{s}$ tends rapidly to zero. Thus one can reduce also to the case where $\left(v_{s}\right)$ and $\left(w_{s}\right)$ are of norm 1 . Then, as $\left(v_{s}\right)$ converges to $v \neq 0,\left(w_{s}\right)$ converges to $v$. One can take $w=v$. Let us look at the canonical map $\varphi$ of the unit sphere of $V$, $S$, to $\mathbf{P}_{\mathbb{R}}(V)$. Applying Lemma B.5, one sees that $\left(w_{s}\right)$ converges rapidly to $w$. Hence $\left(v_{s}\right)$ converges also rapidly to $w$. This implies easily that $\left(\left[v_{s}\right]\right)$ converges rapidly to $[w]$ (cf. end of Remark B.4).

\section{References}

[1] J. N. Bernstein. On the support of Plancherel measure. J. Geom. Phys., 5(4):663-710 (1989), 1988.

[2] J. N. Bernstein and B. Krötz. Smooth Fréchet globalizations of Harish-Chandra modules. Israel Journal of Mathematics, 199(1):45-111, 2014.

[3] A. Borel. Linear algebraic groups, volume 126 of Graduate Texts in Mathematics. Springer-Verlag, New York, second edition, 1991.

[4] J. Carmona. Terme constant des fonctions tempérées sur un espace symétrique réductif. J. Reine Angew. Math., 491:17-63, 1997.

[5] Harish-Chandra. Harmonic analysis on real reductive groups I. The theory of the constant term. J. Funct. Anal., 19:104-204, 1975. 
[6] Harish-Chandra. Harmonic analysis on real reductive groups II. Wave-packets in the Schwartz space. Invent. Math., 36:1-55, 1976.

[7] F. Knop and B. Krötz. Reductive action groups. arXiv:1604.01005v2, 2014.

[8] F. Knop, B. Krötz, E. Sayag, and H. Schlichtkrull. Simple compactifications and polar decomposition of homogeneous real spherical spaces. Selecta Math., 21(3):1071-1097, 2015.

[9] F. Knop, B. Krötz, E. Sayag, and H. Schlichtkrull. Volume growth, temperedness and integrability of matrix coefficients on a real spherical spaces. Selecta Math., 21(3):10711097, 2015.

[10] F. Knop, B. Krötz, and H. Schlichtkrull. The local structure theorem for real spherical varieties. Compositio Mathematica, 151:2145-2159, 2015.

[11] F. Knop, B. Krötz, and H. Schlichtkrull. The tempered spectrum of a real spherical space. arXiv:1509.03429v2, 2016.

[12] B. Krötz and H. Schlichtkrull. Multiplicity bounds and the subrepresentation theorem for real spherical spaces. arXiv:1309.0930v2, 2014.

[13] Y. Sakellaridis and A. Venkatesh. Periods and harmonic analysis on spherical varieties. arXiv:1203.0039v3, 2014.

[14] V. S. Varadarajan. Harmonic analysis on real reductive groups. Lecture Notes in Mathematics, Vol. 576. Springer-Verlag, Berlin-New York, 1977.

[15] N. R. Wallach. Real reductive groups. I, volume 132 of Pure and Applied Mathematics. Academic Press, Inc., Boston, MA, 1988.

[16] N. R. Wallach. Real reductive groups. II, volume 132 of Pure and Applied Mathematics. Academic Press Inc., Boston, MA, 1992.

[17] G. Warner. Harmonic analysis on semi-simple Lie groups. I. Springer-Verlag, New York-Heidelberg, 1972. Die Grundlehren der mathematischen Wissenschaften, Band 188. 